\title{
Graphene Oxide Promotes Site-Selective Allylic Alkylation of Thiophenes With Alcohols.
}

Laura Favaretto, ${ }^{\ddagger}$ Juzeng An, ${ }^{\dagger}$ Marco Sambo, ${ }^{\dagger}$ Assunta De Nisi, ${ }^{\dagger}$ Cristian Bettini, ${ }^{\ddagger}$ Manuela Melucci, ${ }^{\ddagger}$ Alessandro Kovtun, ${ }^{\ddagger}$ Andrea Liscio, ${ }^{\ddagger},{ }^{\prime I}$ Vincenzo Palermo, ${ }^{\ddagger}$ Andrea Bottoni, ${ }^{\dagger}$ Francesco Zerbetto, ${ }^{\dagger}$ Matteo Calvaresi, ${ }^{\dagger}$ Marco Bandini ${ }^{\dagger *}$

${ }^{\dagger}$ Dipartimento di Chimica "G. Ciamician”, Alma Mater Studiorum - Università di Bologna, via Selmi 2, 40126 Bologna, Italy. ${ }^{\ddagger}$ Istituto per la Sintesi Organica e Fotoreattività (ISOF) - CNR, via Gobetti 101, 40129 Bologna, ${ }^{\text {Tl }}$ Istituto per la Microelettronica e Microsistemi (IMM) - CNR, via del Fosso del Cavaliere 100, 00133 Roma.

\section{Table of contents:}

$\begin{array}{ll}\text { General Methods } & \text { S2 }\end{array}$

$\begin{array}{ll}\text { Computational Details } & \text { S2 }\end{array}$

$\begin{array}{ll}\text { X-ray photoelectron spectroscopy (XPS) measurements } & \text { S2 }\end{array}$

Synthesis of the allylic alcohols: $2 \mathbf{2 b - f , j - 1}$ (not optimized protocol) $\quad$ S4

$\begin{array}{ll}\text { Synthesis of aldehyde, precursor alcohol } 2 \mathrm{~h} & \text { S5 }\end{array}$

Typical procedure for the GO-assisted Friedel-Crafts reaction $\quad$ S5

$\begin{array}{ll}\text { Figure } 1 & \text { S12 }\end{array}$

$\begin{array}{ll}\text { Table S1 } & \text { S13 }\end{array}$

$\begin{array}{ll}\text { NMR Spectra } & \text { S14 }\end{array}$

$\begin{array}{ll}\text { Figure S2 } & \text { S37 }\end{array}$

$\begin{array}{lr}\text { Table S2. Cartesian coordinates } & \text { S37 }\end{array}$ 


\section{General Methods.}

${ }^{1} \mathrm{H}-\mathrm{NMR}$ spectra were recorded on Varian 400 (400 MHz) spectrometer or Agilent NMR system, consisting of a 54-mm bore, $500 \mathrm{MHz}$ (11.7 T) Premium Shielded superconducting magnet, a DD2 Performa IV NMR console and the Agilent OneNMR probe. ${ }^{1} \mathrm{H}$ NMR $\left(\mathrm{C}_{6} \mathrm{D}_{6}, 500 \mathrm{MHz}\right) ;{ }^{13} \mathrm{C}$ NMR $\left(\mathrm{C}_{6} \mathrm{D}_{6}, 125 \mathrm{MHz}\right)$. Chemical shifts are reported in ppm from TMS with the solvent resonance as the internal standard (deuterochloroform: $7.26 \mathrm{ppm})$. Data are reported as follows: chemical shift, multiplicity $(\mathrm{s}=$ singlet, $\mathrm{d}=$ duplet, $\mathrm{t}=$ triplet, $\mathrm{q}=$ quartet, $\mathrm{br}=$ broad, $\mathrm{m}=$ multiplet), coupling constants $(\mathrm{Hz}) \cdot{ }^{13} \mathrm{C}-\mathrm{NMR}$ spectra were recorded on a Varian $400(100 \mathrm{MHz})$ spectrometer with complete proton decoupling. Chemical shifts are reported in ppm from TMS with the solvent as the internal standard (deuterochloroform: $77.0 \mathrm{ppm}$ ). GC-MS spectra were taken by EI ionization at $70 \mathrm{eV}$ on a Hewlett-Packard 5971 with GC injection or by Trace $1300 \mathrm{GC}$, ISQ Single Quadrupole MS, Thermo Fisher Scientific, Waltham, MA, USA, operating in electron impact (EI) ionization mode at $70 \mathrm{eV}$. One sample was introduced to the ion source region via a direct exposure probe (DEP). They are reported as: $\mathrm{m} / \mathrm{z}$ (rel. intense). Elemental analyses were carried out by using a EACE 1110 CHNOS analyzer. Graphene oxide is prepared using a modified Hummers method starting from graphite flakes (maximum particle diameter $500 \mu \mathrm{m}){ }^{1}$

LC-electrospray ionization mass spectra were obtained with Agilent Technologies MSD1100 singlequadrupole mass spectrometer. Chromatographic purification was done with 240-400 mesh silica gel. Elemental analyses were carried out by using a EACE 1110 CHNOS analyzer.

All anhydrous solvents were supplied by Sigma Aldrich in Sureseal ${ }^{\circledR}$ bottles and used without any further purification. Commercially available chemicals were purchased from Sigma Aldrich, Stream and TCI and used without any further purification. Melting points were measured using open glass capillaries in a Bibby Stuart Scientific Melting Point Apparatus SMP 3 and are calibrated by comparison with literature values (Aldrich). Alcohols 2a and 2a' were purchased from Merck-Aldrich and used as received.

Certainly, the cost of styrene oxide cannot be compared with that of GO. However, styrene oxide proved to be poorly effective in the titled transformation, leading to 30\% 3aa conversion only when epoxide (100\%) was combined with stoichiometric amount of benzoic acid. Therefore, in the present protocol, GO is much more performing with respect to smaller oxiranes. Additionally, I would like to emphasize that with the present work we wish to present a "proof-of-principle", regarding the application of GO to synthetically useful organic reactions. No intent to replace "classic" metal and metal-free catalytic strategies with GO and derivatives.

\section{Computational Details.}

All calculations were carried out using the combined quantum mechanical and molecular mechanical (QM/MM) method in the ONIOM formalism, as implemented within the Gaussian 09 program suite. ${ }^{2}$ The coupled QM/MM method adopted here is a two-layer ONIOM scheme, where the reactive region is treated at DFT level using the density functional M06-2X (a functional able to account for $\pi$ - $\pi$ interactions) and 6-

\footnotetext{
${ }^{1}$ a) Park, S.; Ruoff, R. S. Nat. Nanotechnol. 2009, 4, 217-224; b) Treossi, E.; Melucci, M.; Liscio, A.; Gazzano, M.; Samorì, P.; Palermo, V. J. Am. Chem. Soc. 2009, 131, 15576-15577.

${ }^{2}$ Gaussian 09, Revision E.01, Frisch, M. J.; Trucks, G. W.; Schlegel, H. B.; Scuseria, G. E.; Robb, M. A.; Cheeseman, J. R.; Scalmani, G.; Barone, V.; Mennucci, B.; Petersson, G. A.; Nakatsuji, H.; Caricato, M.; Li, X.; Hratchian, H. P.; Izmaylov, A. F.; Bloino, J.; Zheng, G.; Sonnenberg, J. L.; Hada, M.; Ehara, M.; Toyota, K.; Fukuda, R.; Hasegawa, J.; Ishida, M.; Nakajima, T.; Honda, Y.; Kitao, O.; Nakai, H.; Vreven, T.; Montgomery, J. A., Jr.; Peralta, J. E.; Ogliaro, F.; Bearpark, M.; Heyd, J. J.; Brothers, E.; Kudin, K. N.; Staroverov, V. N.; Kobayashi, R.; Normand, J.; Raghavachari, K.; Rendell, A.; Burant, J. C.; Iyengar, S. S.; Tomasi, J.; Cossi, M.; Rega, N.; Millam, J. M.; Klene, M.; Knox, J. E.; Cross, J. B.; Bakken, V.; Adamo, C.; Jaramillo, J.; Gomperts, R.; Stratmann, R. E.; Yazyev, O.; Austin, A. J.; Cammi, R.; Pomelli, C.; Ochterski, J. W.; Martin, R. L.; Morokuma, K.; Zakrzewski, V. G.; Voth, G. A.; Salvador, P.; Dannenberg, J. J.; Dapprich, S.; Daniels, A. D.; Farkas, Ö.; Foresman, J. B.; Ortiz, J. V.; Cioslowski, J.; Fox, D. J. Gaussian, Inc., Wallingford CT, 2009.
} 
31G* basis set. ${ }^{3}$ The remaining region is treated using the UFF force field (see Scheme S1 in SI). ${ }^{4}$ The global potential can be referred as M06-2X/6-31G*:UFF potential. ONIOM calculations were performed considering mechanical and electrostatic embedding. In the MM calculations partial atomic (point) charges were used to compute the electrostatic interactions. These charges were calculated using the QEq formalism. ${ }^{5}$ The structure of the various critical points (minima and saddle points) was fully optimized. Frequency calculations were carried out at the same level of theory to check the nature of critical points. This approach was recently validated to study similar carbon based nanocatalyst. ${ }^{6}$

\section{X-ray photoelectron spectroscopy (XPS) measurements.}

XPS spectra were recorded with a Phoibos 100 hemispherical energy analyzer (Specs) using $\mathrm{Mg} \mathrm{K}_{\alpha}$ radiation $(\hbar \omega=1253.6 \mathrm{eV})$. The $\mathrm{X}$-ray power was $125 \mathrm{~W}$. The spectra were recorded in the constant analyzer energy (CAE) mode with analyzer pass energies of $40 \mathrm{eV}$ for the high resolution spectra. Charging effects were corrected by energy calibration on $\mathrm{Au} 4 \mathrm{f}_{7 / 2}$ level relative to $84.0 \mathrm{eV}$. The base pressure in the analysis chamber during analysis was $5 \times 10^{-10}$ mbar. High resolution XPS spectra of $\mathrm{C} 1 \mathrm{~s}$ were analyzed by CasaXPS (Casa software, Ltd), the curve fitting was carried out using Gaussian/Lorentzian curves shape (GL(30)) for C-O groups with a full width half-maximum of $1.4 \mathrm{eV}$ and an asymmetric Voigt for the C-C sp2.

For each sample GO were deposited on golden slides purchased from Arrandee (Germany) having the following specifications: $\mathrm{Au}, 250 \mathrm{~nm}$ thick and $2.5 \mathrm{~nm}$ of $\mathrm{Cr}$, as adhesive layer. Gold substrates were cleaned using the protocol for silicon substrates. Sample were prepared drop-casting the GO solutions on freshly cleaned golden slice and immediately put in High-Vacuum overnight.

\footnotetext{
${ }^{3}$ Zhao, Y.; Truhlar, D. G. Theor. Chem. Acc. 2008, 120, 215-241.

${ }^{4}$ Rappé, A. K.; Casewit, C. J.; Colwell, K. S.; Goddard, W. A., III; Skiff, W. M. J. Am. Chem. Soc. 1992, 114, 10024-10035.

${ }^{5}$ Rappé, A. K.; Goddard, W. A., III J. Phys. Chem. 1991, 95, 3358-3363.

${ }^{6}$ a) Giacinto, P.; Bottoni, A.; Calvaresi, M.; Zerbetto, F. J. Phys. Chem. C 2014, 118, 5032-5040; b) Spyrou, K.; Calvaresi, M.; Diamanti, E. K.; Tsoufis, T.; Gournis, D.; Rudolf, P.; Zerbetto, F. Adv. Funct. Mater. 2015, 25, 263-269; c) Giacinto, P.; Zerbetto, F.; Bottoni, A.; Calvaresi, M. J. Chem. Theory Comput. 2016, 12, 4082-4092; d) Marforio, T. D.; Bottoni, A.; Giacinto, P.; Zerbetto, F.; Calvaresi, M. J. Phys. Chem. $C, \mathbf{2 0 1 7}, 121,27674-27682$
} 
Synthesis of the allylic alcohols: $2 \mathrm{~b}-\mathbf{f}, \mathbf{j}-\mathbf{l}$ (not optimized protocol).

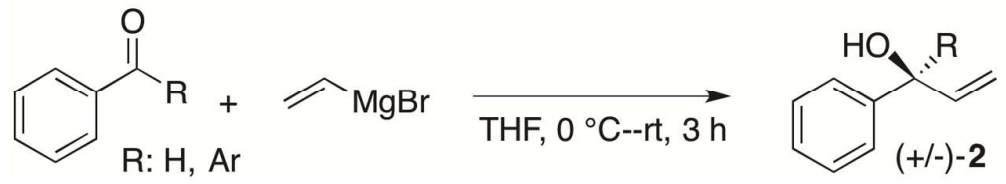

A flame-dried three-necked round bottom flask was charged under nitrogen with anhydrous THF followed by the desired carbonyl compound $(3 \mathrm{mmol}, 0.2 \mathrm{M})$ and the mixture was cooled to $0{ }^{\circ} \mathrm{C}$. Therefore, a solution of vinylmagnesium bromide (1M, $1.5 \mathrm{eq})$ was added dropwise, followed by stirring at $\mathrm{rt}$ for 3 hours. The reaction was quenched with an aqueous $\mathrm{NH}_{4} \mathrm{Cl}$ solution $(2 \mathrm{M})$, extracted with $\mathrm{Et}_{2} \mathrm{O}(3 \times 15 \mathrm{~mL})$ and the combined organic phases dried over $\mathrm{Na}_{2} \mathrm{SO}_{4}$. Solvent was removed by rotary evaporation and the crude was purified by column chromatography to give the desired product.

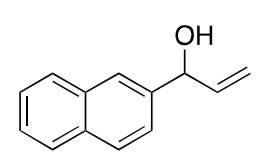

2b. ${ }^{7}$ Yellow oil. Yield $=54 \%(300 \mathrm{mg}) .\left(c \mathrm{Hex}_{\mathrm{Et}} \mathrm{O}=85: 15\right) .{ }^{1} \boldsymbol{H}-\boldsymbol{N M R}(500 \mathrm{MHz}$, $\left.\mathrm{CDCl}_{3}\right)$ : $7.85-7.83(\mathrm{~m}, 4 \mathrm{H}), 7.50-7.48(\mathrm{~m}, 3 \mathrm{H}), 6.17-6.10(\mathrm{~m}, 1 \mathrm{H}), 5.42(\mathrm{~d}, \mathrm{~J}=17.5$ $\mathrm{Hz}, 1 \mathrm{H}), 5.38(\mathrm{~d}, \mathrm{~J}=5.5 \mathrm{~Hz}, 1 \mathrm{H}), 5.25(\mathrm{~d}, \mathrm{~J}=10.0 \mathrm{~Hz}, 1 \mathrm{H}), 2.03$ (bs, $1 \mathrm{H}) .{ }^{13} \boldsymbol{C}-\boldsymbol{N M R}$

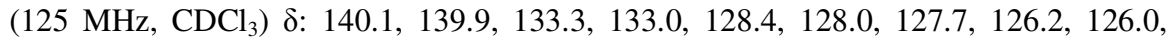
124.9, 124.5, 115.4, 75.5. $\boldsymbol{G C}$-MS (m/z): 128, 184.

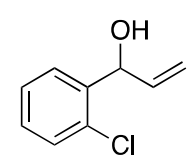

2c. ${ }^{8}$ Colorless oil. Yield $=44 \%(222 \mathrm{mg}),\left(c \mathrm{Hex}_{\mathrm{Et}} \mathrm{Et}_{2} \mathrm{O}=85: 15\right) .{ }^{1} \boldsymbol{H}-\mathbf{N M R}(500 \mathrm{MHz}$, $\left.\mathrm{CDCl}_{3}\right) \delta: 7.53(\mathrm{dd}, \mathrm{J}=1.5 \mathrm{~Hz}, \mathrm{~J}=8.0 \mathrm{~Hz}, 1 \mathrm{H}), 7.34(\mathrm{~d}, \mathrm{~J}=8.0 \mathrm{~Hz}, 1 \mathrm{H}), 7.28(\mathrm{t}, \mathrm{J}=7.5$ $\mathrm{Hz}, 1 \mathrm{H}), 7.21(\mathrm{dt}, \mathrm{J}=1.5 \mathrm{~Hz}, \mathrm{~J}=7.5 \mathrm{~Hz}, 1 \mathrm{H}), 6.05-5.99(\mathrm{~m}, 1 \mathrm{H}), 5.62(\mathrm{~d}, \mathrm{~J}=5.5 \mathrm{~Hz}$, 1H), $5.37(\mathrm{~d}, \mathrm{~J}=17.0 \mathrm{~Hz}, 1 \mathrm{H}), 5.21(\mathrm{~d}, \mathrm{~J}=10.5 \mathrm{~Hz}, 1 \mathrm{H}), 2.45$ (bs, $1 \mathrm{H}) .{ }^{13} \boldsymbol{C}-\boldsymbol{N M R}(125$ $\left.\mathrm{MHz}, \mathrm{CDCl}_{3}\right) \delta: 139.9,138.3,132.3,129.4,128.7,127.6,127.1,115.5,71.3 . \mathbf{G C}-\mathbf{M S}$ $(\mathrm{m} / \mathrm{z}): 168,170$.

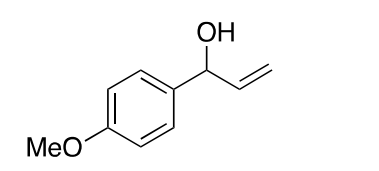

2d. ${ }^{9}$ Colorless oil. Yield $=64 \%(314 \mathrm{mg}),\left(c \mathrm{Hex} \mathrm{Et}_{2} \mathrm{O}=80: 20\right) .{ }^{1} \boldsymbol{H}-\boldsymbol{N M R}(400 \mathrm{MHz}$, $\left.\mathrm{CDCl}_{3}\right) \delta 7.28(\mathrm{~d}, \mathrm{~J}=8.0 \mathrm{~Hz}, 2 \mathrm{H}), 6.89(\mathrm{~d}, \mathrm{~J}=8.0 \mathrm{~Hz}, 2 \mathrm{H}), 6.08-5.98(\mathrm{~m}, 1 \mathrm{H}), 5.32(\mathrm{~d}, \mathrm{~J}$ $=18.4 \mathrm{~Hz}, 1 \mathrm{H}), 5.18(\mathrm{~d}, \mathrm{~J}=10.3 \mathrm{~Hz}, 1 \mathrm{H}), 5.13(\mathrm{brs}, 1 \mathrm{H}), 3.78(\mathrm{~s}, 3 \mathrm{H}), 2.16(\mathrm{brs}, 1 \mathrm{H})$. ${ }^{13} \boldsymbol{C}-\mathrm{NMR}\left(100 \mathrm{MHz}, \mathrm{CDCl}_{3}\right) \delta 159.14,140.4,134.9,127.7(2 \mathrm{C}), 114.7,113.9$ (2C), 74.8, 55.3. $\boldsymbol{G C}-\boldsymbol{M S}(\mathrm{m} / \mathrm{z}):$ 77, 109, 164.

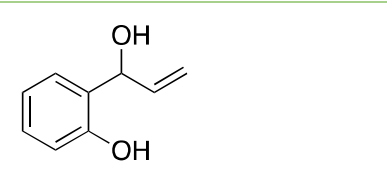

2e. ${ }^{10}$ Colorless oil. Yield $=35 \%(158 \mathrm{mg}),\left(c \mathrm{Hex}_{\mathrm{Et}} \mathrm{Et} \mathrm{O}=80: 20\right) .{ }^{1} \boldsymbol{H}$ - $\boldsymbol{N M R}(400 \mathrm{MHz}$, $\left.\mathrm{CDCl}_{3}\right) \delta 7.66(\mathrm{~s}, 1 \mathrm{H}), 7.19(\mathrm{dt}, \mathrm{J}=1.6 \mathrm{~Hz}, \mathrm{~J}=8.4 \mathrm{~Hz}, 1 \mathrm{H}), 7.28(\mathrm{dd}, \mathrm{J}=1.2 \mathrm{~Hz}, \mathrm{~J}=7.6$ $\mathrm{Hz}, 1 \mathrm{H}), 6.89-6.84(\mathrm{~m}, 2 \mathrm{H}), 6.22-6.10(\mathrm{~m}, 1 \mathrm{H}), 5.39(\mathrm{brs}, 1 \mathrm{H}), 5.35(\mathrm{~d}, \mathrm{~J}=17.2 \mathrm{~Hz}$, $1 \mathrm{H}), 5.27(\mathrm{~d}, \mathrm{~J}=17.2 \mathrm{~Hz}, 1 \mathrm{H}), 2.65(\mathrm{~s}, 1 \mathrm{H}) .{ }^{13} \boldsymbol{C}-\mathbf{N M R}\left(100 \mathrm{MHz}, \mathrm{CDCl}_{3}\right) \delta 155.3$, 138.0, 129.3, 127.6, 120.0, 117.1, 116.3, 76.1. GC-MS (m/z): 150 .

2f. ${ }^{11}$ Colorless oil. Yield $=54 \%(343 \mathrm{mg}),\left(n\right.$-Pent:EtOAc: $\mathrm{CH}_{2} \mathrm{Cl}_{2}=$ 90:5:5 $\rightarrow$ 60:20:20).

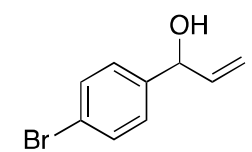

${ }^{I} \boldsymbol{H}-\mathbf{N M R}\left(500 \mathrm{MHz}, \mathrm{CDCl}_{3}\right) \delta: 7.48(\mathrm{dd}, \mathrm{J}=3.0 \mathrm{~Hz}, \mathrm{~J}=8.5 \mathrm{~Hz}, 2 \mathrm{H}), 7.25(\mathrm{dd}, \mathrm{J}=3.0$ $\mathrm{Hz}, \mathrm{J}=8.5 \mathrm{~Hz}, 2 \mathrm{H}), 6.04-5.97(\mathrm{~m}, 1 \mathrm{H}), 5.34(\mathrm{dd}, \mathrm{J}=1.5 \mathrm{~Hz}, \mathrm{~J}=17.0 \mathrm{~Hz}, 1 \mathrm{H}), 5.23-5.21$ (m, 1H), 5.17 (bs, 1H), 2.01 (bs, 1H). ${ }^{13} \boldsymbol{C}-\boldsymbol{N M R}\left(125 \mathrm{MHz}, \mathrm{CDCl}_{3}\right)$ 8: 141.5, 139.8, 131.6, 128.0, 121.5, 115.6, 74.7. $\boldsymbol{G C}$-MS (m/z): 133, 183-185, 212-214.

2g. Yellow oil. Yield $=96 \%(541 \mathrm{mg}),\left(c \mathrm{Hex}_{\mathrm{Et}} \mathrm{O}=90: 10\right) .{ }^{1} \boldsymbol{H}-\boldsymbol{N M R}(400 \mathrm{MHz}$,

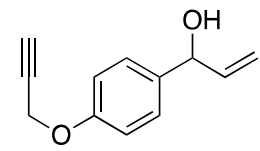
$\left.\mathrm{CDCl}_{3}\right) \delta 7.31(\mathrm{~d}, \mathrm{~J}=8.8 \mathrm{~Hz}, 2 \mathrm{H}), 6.97(\mathrm{~d}, \mathrm{~J}=8.8 \mathrm{~Hz}, 2 \mathrm{H}), 6.00-6.09(\mathrm{~m}, 1 \mathrm{H}), 5.34(\mathrm{dt}, \mathrm{J}$ $=1.2,17.2 \mathrm{~Hz}, 1 \mathrm{H}), 5.19(\mathrm{dt}, \mathrm{J}=1.2 \mathrm{~Hz}, 12.6 \mathrm{~Hz}, 1 \mathrm{H}), 5.16(\mathrm{brs}, 1 \mathrm{H}), 4.69(\mathrm{~d}, \mathrm{~J}=2.4$ $\mathrm{Hz}, 2 \mathrm{H}), 2.55(\mathrm{t}, \mathrm{J}=2.8 \mathrm{~Hz}, 1 \mathrm{H}) .{ }^{13} \boldsymbol{C}-\boldsymbol{N M R}\left(100 \mathrm{MHz}, \mathrm{CDCl}_{3}\right) \delta 157.1,140.2,135.8$, 127.7(2C), 114.9(2C), 114.8, 78.5, 75.5, 74.8, 55.8. GC-MS (m/z): 188. Anal. Calc. for $\left(\mathrm{C}_{12} \mathrm{H}_{12} \mathrm{O}_{2}\right.$ : 188.08): C, 76.57; H, 6.43; found: C, 77.41, H, 6.55 .

\footnotetext{
${ }^{7}$ M. Lafrance, M. Roggen, E. M. Carreira, Angew. Chem. Int. Ed. 2012, 51, 3470-3473.

${ }^{8}$ S. F. Musolino, O. S. Ojo, N. J. Westwood, J. E. Taylor, A. D. Smith, Chem. Eur. J. 2016, 22, 18916-18922.

${ }^{9}$ F. Wu, H. Li, R. Hong, L. Deng, Angew. Chem. Int. Ed. 2006, 45, 947-950.

${ }^{10}$ M. Vellakkaran, M. M. S. Andappan, N. Kommu, Eur. J. Org. Chem. 2012, 4694-4698.

${ }^{11}$ J. Štambaský, A. V. Malkov, P. Kočovský, J. Org. Chem. 2008, 73, 9148-9150.
} 
2h. Pale yellow oil. Yield $=57 \%(446 \mathrm{mg}),(c \mathrm{Hex}:$ EtOAc $=80: 20) .{ }^{1} \boldsymbol{H}-\mathbf{N M R}(400 \mathrm{MHz}$, $\left.\mathrm{CDCl}_{3}\right) \delta 7.26(\mathrm{q}, \mathrm{J}=1.6 \mathrm{~Hz}, \mathrm{~J}=6.4 \mathrm{~Hz}, 2 \mathrm{H}), 6.86(\mathrm{q}, \mathrm{J}=2.0 \mathrm{~Hz}, \mathrm{~J}=6.8 \mathrm{~Hz}, 2 \mathrm{H}), 6.03$ $(\mathrm{ddd}, \mathrm{J}=5.6 \mathrm{~Hz}, \mathrm{~J}=10.0 \mathrm{~Hz}, \mathrm{~J}=16.4 \mathrm{~Hz}, 1 \mathrm{H}), 5.33(\mathrm{~d}, \mathrm{~J}=16.4 \mathrm{~Hz}, 1 \mathrm{H}), 5.17$ (d, J = 5.6 $\mathrm{Hz}, 1 \mathrm{H}), 5.15(\mathrm{~d}, \mathrm{~J}=5.6 \mathrm{~Hz}, 1 \mathrm{H}), 3.95(\mathrm{t}, \mathrm{J}=6.0 \mathrm{~Hz}, 2 \mathrm{H}), 3.28(\mathrm{t}, \mathrm{J}=6.8 \mathrm{~Hz}, 2 \mathrm{H}), 1.95$ (brs, $1 \mathrm{H}), 1.83-1.76(\mathrm{~m}, 2 \mathrm{H}), 1.68-1.62(\mathrm{~m}, 2 \mathrm{H}), 1.58-1.54(\mathrm{~m}, 2 \mathrm{H}) .{ }^{13} \boldsymbol{C}$-NMR $(100$ $\left.\mathrm{MHz}_{\mathrm{CDCl}}\right) \delta 158.6,140.4,134.8,127.7(2 \mathrm{C}), 114.7,114.5(2 \mathrm{C}), 114.9$, 74.9, 67.6, 51.3, 28.8, 28.6, 23.4. LC-MS: 206, 248. Anal. Calc. for $\left(\mathrm{C}_{14} \mathrm{H}_{19} \mathrm{~N}_{3} \mathrm{O}_{2}:\right.$ : 261.15): C, 64.35; H, 7.33; found: C, 64.22, H, 7.21.

21. Yellow solid. $\boldsymbol{M P}=93-95{ }^{\circ} \mathrm{C}$. Yield $=43 \%(268 \mathrm{mg}),(c$ Hex:EtOAc $=90: 10) .{ }^{\mathbf{I}} \boldsymbol{H}$ -

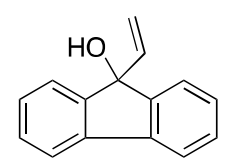
NMR $\left(500 \mathrm{MHz}, \mathrm{CDCl}_{3}\right) \delta: 7.64(\mathrm{~d}, \mathrm{~J}=7.0 \mathrm{~Hz}, 2 \mathrm{H}), 7.46(\mathrm{~d}, \mathrm{~J}=7.0 \mathrm{~Hz}, 2 \mathrm{H}), 7.38(\mathrm{t}, \mathrm{J}=$ $7.5 \mathrm{~Hz}, 2 \mathrm{H}), 7.31(\mathrm{t}, \mathrm{J}=7.5 \mathrm{~Hz}, 2 \mathrm{H}), 5.99(\mathrm{dd}, \mathrm{J}=11.0 \mathrm{~Hz}, \mathrm{~J}=17.5 \mathrm{~Hz}, 1 \mathrm{H}), 5.56(\mathrm{~d}, \mathrm{~J}=$ $17.0 \mathrm{~Hz}, 1 \mathrm{H}), 5.22(\mathrm{~d}, \mathrm{~J}=11.0 \mathrm{~Hz}, 1 \mathrm{H}), 2.20$ (bs, $1 \mathrm{H}) .{ }^{13} \boldsymbol{C}-\boldsymbol{N M R}\left(125 \mathrm{MHz}, \mathrm{CDCl}_{3}\right) \delta$ : $148.0,139.5,139.1,129.2,128.1,124.5,120.2,113.5,82.5 . G C-M S(\mathrm{~m} / \mathrm{z}): 152,181$, 208.

2m. Pale yellow solid. $\boldsymbol{M P}=96-97{ }^{\circ} \mathrm{C}$. Yield $=77 \%(600 \mathrm{mg}),(c \mathrm{Hex}:$ EtOAc $=80: 20)$. ${ }^{1} \boldsymbol{H}$-NMR $\left(500 \mathrm{MHz}, \mathrm{CDCl}_{3}\right) \delta: 8.40(\mathrm{~d}, \mathrm{~J}=9.5 \mathrm{~Hz}, 1 \mathrm{H}), 8.20-8.15(\mathrm{~m}, 4 \mathrm{H}), 8.11(\mathrm{~d}, \mathrm{~J}=$ $9.5 \mathrm{~Hz}, 1 \mathrm{H}), 8.07-8.00(\mathrm{~m}, 3 \mathrm{H}), 6.39-6.32(\mathrm{~m}, 1 \mathrm{H}), 6.27(\mathrm{~d}, \mathrm{~J}=4.5 \mathrm{~Hz}, 1 \mathrm{H}), 5.48(\mathrm{~d}, \mathrm{~J}=$ $17.0 \mathrm{~Hz}, 1 \mathrm{H}), 5.31(\mathrm{~d}, \mathrm{~J}=10.0 \mathrm{~Hz}, 1 \mathrm{H}), 2.27(\mathrm{bs}, 1 \mathrm{H}) .{ }^{13} \boldsymbol{C}-\boldsymbol{N M R}\left(125 \mathrm{MHz}, \mathrm{CDCl}_{3}\right) \delta$ : 139.9, 135.6, 131.3, 131.0, 130.7, 128.1, 127.7, 127.5, 127.4, 126.0, 125.3, 125.2, 125.0, 124.8, 124.1, 122.9, 115.6, 72.4. GC-MS (m/z): 203, 227, 239, 258.

\section{Synthesis of aldehyde, precursor alcohol $2 \mathrm{~h}$ :}

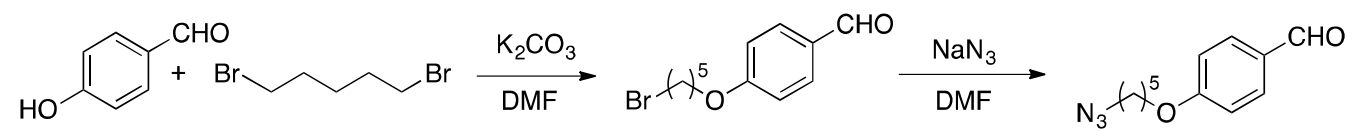

In a $100 \mathrm{~mL}$ Schlenk tube, DMF $(27 \mathrm{~mL})$ was added followed by 4-hydroxybenzaldehyde $(563 \mathrm{mg}, 4.6 \mathrm{mmol})$ and $\mathrm{K}_{2} \mathrm{CO}_{3}(1.27 \mathrm{~g}, 9.2 \mathrm{mmol})$. Then 1,5-dibromopentane $(1.25 \mathrm{~mL}, 9.2 \mathrm{mmol})$ was added at $0{ }^{\circ} \mathrm{C}$. Then the reaction mixture was stirred at room temperature for $12 \mathrm{~h}$. Then the reaction mixture was diluted with EtOAc and washed 3 times with $\mathrm{H}_{2} \mathrm{O}$. The organic phase was dried with $\mathrm{Na}_{2} \mathrm{SO}_{4}$ and the solvent removed under reduced pressure. The reaction crude was purified via flash chromatography $(c \mathrm{Hex}$ :AcOEt $=98: 2)$, to give the desired product in $90 \%$ yield $(1180 \mathrm{mg}) .{ }^{12}$ Yellow oil. ${ }^{\mathbf{I}} \boldsymbol{H}$-NMR $\left(400 \mathrm{MHz} \mathrm{CDCl}_{3}\right) \delta 9.85(\mathrm{~s}, 1 \mathrm{H}), 7.80(\mathrm{dd}, \mathrm{J}=2.4 \mathrm{~Hz}, \mathrm{~J}=4.8 \mathrm{~Hz}, 2 \mathrm{H}), 6.97(\mathrm{dd}, \mathrm{J}=$ $2.8 \mathrm{~Hz}, \mathrm{~J}=5.6 \mathrm{~Hz}, 2 \mathrm{H}), 4.04(\mathrm{t}, \mathrm{J}=6.4 \mathrm{~Hz}, 2 \mathrm{H}), 3.32(\mathrm{t}, \mathrm{J}=6.4 \mathrm{~Hz}, 2 \mathrm{H}), 1.96-1.90(\mathrm{~m}, 2 \mathrm{H}), 1.88-1.79(\mathrm{~m}, 2 \mathrm{H}), 1.64-$ 1.60 (m, 2H). ${ }^{13} \boldsymbol{C}$-NMR $\delta$ 190.7, 164.0, 131.9(2C), 129.8, 114.7(2C), 67.9, 33.4, 32.3, 28.2, 24.7. GC-MS (m/z): 69, 121, 149, 151, 270, 272. Anal. Calc. for $\left(\mathrm{C}_{12} \mathrm{H}_{15} \mathrm{BrO}_{2}\right.$ : 271.15): C, 53.16; H, 5.58; found: C, 53.01, H, 5.75.

A round bottom flask was charged with the 4-(5-bromopentyloxy) benzaldehyde $(250 \mathrm{mg}, 0.92 \mathrm{mmol})$ and $15 \mathrm{~mL}$ of reagent grade DMF and $120 \mathrm{mg}$ of $\mathrm{NaN}_{3}(1.84 \mathrm{mmol})$ was added. The reaction mixture was stirred reflux condition for 16 hours, then the mixture was diluted with $\mathrm{E}_{2} \mathrm{O}(15 \mathrm{~mL})$ and washed with $\mathrm{H}_{2} \mathrm{O}(3 \times 10 \mathrm{~mL})$. Solvent was removed by rotary evaporation and the crude was purified via flash chromatography $(c$ Hex: EtOAc $=98: 2)$. Yield $63 \%(135 \mathrm{mg})$. Dark green oil. ${ }^{1} \boldsymbol{H}-\mathbf{N M R}\left(400 \mathrm{MHz}, \mathrm{CDCl}_{3}\right) \delta 9.87(\mathrm{~s}, 1 \mathrm{H}), 7.80(\mathrm{dd}, \mathrm{J}=2.4 \mathrm{~Hz}, \mathrm{~J}=4.4 \mathrm{~Hz}, 2 \mathrm{H}), 6.98(\mathrm{dd}, \mathrm{J}=2.4 \mathrm{~Hz}, \mathrm{~J}$ $=4.4 \mathrm{~Hz}, 2 \mathrm{H}), 4.04(\mathrm{t}, \mathrm{J}=6.4 \mathrm{~Hz}, 2 \mathrm{H}), 3.30(\mathrm{t}, \mathrm{J}=6.4 \mathrm{~Hz} 1 \mathrm{H}), 1.87-1.82(\mathrm{~m}, 2 \mathrm{H}), 1.71-1.65(\mathrm{~m}, 2 \mathrm{H}), 1.60-1.55(\mathrm{~m}$, 2H). ${ }^{13}$ C-NMR (100 MHz, $\left.\mathrm{CDCl}_{3}\right) \delta$ 190.7, 164.0. 132.0(2C), 129.9, 114.7(2C), 68.0, 51.3. 28.6, 28.5, 23.3. LC-MS (m/z): $234\left[\mathrm{M}+\mathrm{H}^{+}\right]$. Anal. Calc. for $\left(\mathrm{C}_{12} \mathrm{H}_{15} \mathrm{~N}_{3} \mathrm{O}_{2}\right.$ : 233.27): C, 61.79; H, 6.48; found: C, 61.59, H, 6.32.

\section{Typical procedure for the GO-assisted Friedel-Crafts reaction.}

In a typical 2-methylthiophene $(29.0 \mu \mathrm{L}, 0.3 \mathrm{mmol})$ was dissolved in $0.3 \mathrm{~mL}$ of reagent grade 1,4 -dioxane in which, 3.2 $\mathrm{mg}$ of GO (25-50 wt\%) were previously suspended. Then, $\alpha$-vinylbenzyl alcohol $(13.0 \mu \mathrm{L}, 0.1 \mathrm{mmol})$ was added. The mixture was stirred at $90{ }^{\circ} \mathrm{C}$ for 8 hours. Then, the catalyst was filtered and washed with EtOAc and $\mathrm{CH}_{2} \mathrm{Cl}_{2}$. The

\footnotetext{
${ }^{12}$ A. Alizadeh, M. M. Khodaei, A. Eshghi, J. Org. Chem. 2010, 75, 8295.
} 
organic phase was evaporated under reduced pressure and the crude purified by column chromatography to give the desired product.

\section{Typical procedure for the GO-assisted Friedel-Crafts reaction on $1 \mathrm{mmol}$ scale of $2 \mathrm{a}$.}

In a typical 2-methylthiophene $(290 \mu \mathrm{L}, 3.0 \mathrm{mmol})$ was dissolved in $2.5 \mathrm{~mL}$ of reagent grade 1,4-dioxane in which, 32 $\mathrm{mg}$ of GO (25 wt $\%)$ were previously suspended. Then, $\alpha$-vinylbenzyl alcohol $(130 \mu \mathrm{L}, 1.0 \mathrm{mmol})$ was added. The mixture was stirred at $90{ }^{\circ} \mathrm{C}$ for 8 hours. Then, the catalyst was filtered and washed with EtOAc and $\mathrm{CH}_{2} \mathrm{Cl}_{2}$. The organic phase was evaporated under reduced pressure and the crude purified by column chromatography $\left(c \mathrm{Hex}_{\mathrm{E}} \mathrm{Et}_{2} \mathrm{O}=\right.$ 98:2) to give a 3aa+3aa' mixture (86:14) as an yellow oil.

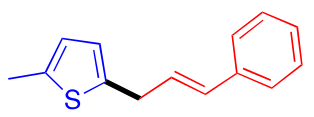

Yield = 90\% (192 mg). ${ }^{1} \boldsymbol{H}-\boldsymbol{N M R}\left(400 \mathrm{MHz}, \mathrm{CDCl}_{3}\right) \delta$ 7.42-7.16 (m, 5H), 6.65-6.58 (m, 2H), $6.48(\mathrm{~d}, \mathrm{~J}=16.0 \mathrm{~Hz}, 1 \mathrm{H})$, $6.34(\mathrm{dt}, \mathrm{J}=5.3 \mathrm{~Hz}, \mathrm{~J}=14.8 \mathrm{~Hz}, 1 \mathrm{H}), 3.64(\mathrm{~d}, \mathrm{~J}=7.2 \mathrm{~Hz}, 2 \mathrm{H}), 2.43(\mathrm{~s}, 3 \mathrm{H}) .{ }^{13} \boldsymbol{C}-\boldsymbol{N M R}\left(100 \mathrm{MHz}, \mathrm{CDCl}_{3}\right) \delta 138.2$, 137.3, 131.1, 128.5 (2C), 127.2, 126.2 (2C), 124.8, 124.4, 121.1, 33.5, 15.3. GC-MS (m/z): 214. Anal. Calc. for $\left(\mathrm{C}_{14} \mathrm{H}_{14} \mathrm{~S}: 214.08\right)$ : C, 78.46; H, 6.58; found: C, 78.21, H, 6.33.

3aa' ${ }^{1} \boldsymbol{H}$-NMR $\left(400 \mathrm{MHz}, \mathrm{CDCl}_{3}\right.$, diagnostic signals) $\delta 7.01(\mathrm{~d}, \mathrm{~J}=4.8 \mathrm{~Hz}, 1 \mathrm{H}), 6.84(\mathrm{~d}, \mathrm{~J}=4.8 \mathrm{~Hz}, 1 \mathrm{H}), 6.38-6.24(\mathrm{~m}$, $2 \mathrm{H}), 3.43(\mathrm{~d}, \mathrm{~J}=5.6 \mathrm{~Hz}, 2 \mathrm{H}), 2.41(\mathrm{~s}, 3 \mathrm{H}) .{ }^{13} \boldsymbol{C}-\mathbf{N M R}\left(100 \mathrm{MHz}, \mathrm{CDCl}_{3}\right.$, diagnostic signals) $\delta$ 130.5, 129.1, 128.4 , $127.0,126.2,126.0,31.8,12.2$.

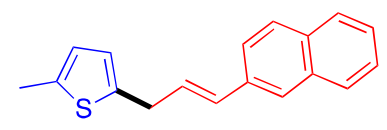

3ab+3ab'+ $\boldsymbol{b}$-3ab $(86: 11: 3)$. White solid. Yield $=69 \%(18 \mathrm{mg})$, (Pent: $\left.\mathrm{CH}_{2} \mathrm{Cl}_{2}=97: 3\right) . \boldsymbol{M P}=38-55{ }^{\circ} \mathrm{C} .{ }^{\boldsymbol{I}} \boldsymbol{H}-\boldsymbol{N M R}(500$ $\left.\mathrm{MHz} \mathrm{CDCl}_{3}\right) \delta$ 7.80-7.77 (m, 3H), $7.72(\mathrm{~s}, 1 \mathrm{H}), 7.60(\mathrm{~d}, \mathrm{~J}=8.5 \mathrm{~Hz}, 1 \mathrm{H}), 7.47-7.43(\mathrm{~m}, 2 \mathrm{H}), 6.68-6.61(\mathrm{~m}, 3 \mathrm{H}), 6.54-$ $6.45(\mathrm{~m}, 1 \mathrm{H}), 3.72(\mathrm{~d}, \mathrm{~J}=7.0 \mathrm{~Hz}, 2 \mathrm{H}), 2.46(\mathrm{~s}, 3 \mathrm{H}) .{ }^{13} \boldsymbol{C}-\boldsymbol{N M R}\left(125 \mathrm{MHz}, \mathrm{CDCl}_{3}\right) \delta$ 140.7, 138.3, 134.8, 133.6, 132.9, 131.2, 128.9, 128.1, 127.9, 127.6, 126.2, 125.9, 125.7, 124.9, 124.5, 123.6, 33.7, 15.4. GC-MS (m/z): 165, $249,264$.

3ab' ${ }^{1} \boldsymbol{H}$-NMR $\left(500 \mathrm{MHz}, \mathrm{CDCl}_{3}\right.$, diagnostic signals $) \delta 7.69(\mathrm{~s}, 1 \mathrm{H}), 7.05(\mathrm{~d}, \mathrm{~J}=5.5 \mathrm{~Hz}, 1 \mathrm{H}), 6.90(\mathrm{~d}, \mathrm{~J}=5.5 \mathrm{~Hz}, 1 \mathrm{H})$, $3.51(\mathrm{~d}, \mathrm{~J}=6.5 \mathrm{~Hz}, 2 \mathrm{H}), 2.43$ (s, 3H). Anal. Calc. for $\left(\mathrm{C}_{18} \mathrm{H}_{16} \mathrm{~S}: 264.10\right)$ : C, 81.77; H, 6.10; found: C, 81.62, H, 6.00. b-3ab ${ }^{1} \boldsymbol{H}$-NMR $\left(500 \mathrm{MHz}, \mathrm{CDCl}_{3}\right.$, diagnostic signals) $\delta 6.38-6.32(\mathrm{~m}, 1 \mathrm{H}), 5.26(\mathrm{~d}, \mathrm{~J}=9.5 \mathrm{~Hz}, 1 \mathrm{H}), 5.16(\mathrm{~d}, \mathrm{~J}=17.0$ $\mathrm{Hz}, 1 \mathrm{H}), 5.00(\mathrm{~d}, \mathrm{~J}=7.0 \mathrm{~Hz}, 1 \mathrm{H})$.

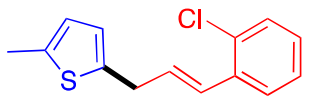

3ac+3ac'+b-3ac (60:27:13). Yellow oil. Yield $=69 \%(17 \mathrm{mg}),\left(c \mathrm{Hex}^{\prime}: \mathrm{Et}_{2} \mathrm{O}=95: 5\right) .{ }^{\boldsymbol{I}} \boldsymbol{H}-\boldsymbol{N M \boldsymbol { R }}\left(400 \mathrm{MHz}, \mathrm{CDCl}_{3}\right) \delta 7.59$ $(\mathrm{dd}, \mathrm{J}=1.6,7.6 \mathrm{~Hz}, 1 \mathrm{H}), 7.37-7.33(\mathrm{~m}, 1 \mathrm{H}), 7.23-7.14(\mathrm{~m}, 3 \mathrm{H}), 6.92-6.89(\mathrm{~m}, 1 \mathrm{H}), 6.65(\mathrm{~d}, \mathrm{~J}=3.2 \mathrm{~Hz}, 1 \mathrm{H}), 6.60$ (brs, 1H), 6.4-6.2 (m, 1H), $3.71(\mathrm{~d}, \mathrm{~J}=7.2 \mathrm{~Hz}, 2 \mathrm{H}), 2.43(\mathrm{~s}, 3 \mathrm{H}) .{ }^{13} \boldsymbol{C}-\boldsymbol{N M R}\left(100 \mathrm{MHz}, \mathrm{CDCl}_{3}\right) \delta$ 140.3, 138.3, 135.4, 131.2, 129.6, 128.2, 127.4, 126.8, 126.7, 124.9, 124.5, 121.2, 33.7, 15.3. GC-MS (m/z): 115, 213, 233, 248.

3ac' ${ }^{1} \boldsymbol{H}$-NMR $\left(400 \mathrm{MHz}, \mathrm{CDCl}_{3}\right.$, diagnostic signals) $\delta 7.49(\mathrm{dd}, \mathrm{J}=6.0 \mathrm{~Hz}, 7.2 \mathrm{~Hz}, 1 \mathrm{H}), 7.03(\mathrm{~d}, \mathrm{~J}=5.2 \mathrm{~Hz}, 1 \mathrm{H}), 3.50$ $(\mathrm{d}, \mathrm{J}=6.5 \mathrm{~Hz}, 2 \mathrm{H}), 2.40$ (s, 3H). ${ }^{13} \mathbf{C}-\mathbf{N M R}\left(100 \mathrm{MHz}, \mathrm{CDCl}_{3}, \mathrm{CDCl}_{3}\right.$, diagnostic signals $) \delta 138.8,134.8,129.5,32.1$, 12.9. Anal. Calc. for $\left(\mathrm{C}_{14} \mathrm{H}_{13} \mathrm{ClS}\right.$ : 248.04): C, 67.59; H, 5.27; found: C, 67.41, H, 5.15.

$\boldsymbol{b}$-3ac' ${ }^{1} \boldsymbol{H}$-NMR $\left(400 \mathrm{MHz}, \mathrm{CDCl}_{3}\right.$, diagnostic signals) $\delta 5.54(\mathrm{~d}, \mathrm{~J}=6.6 \mathrm{~Hz}, 2 \mathrm{H}), 5.21(\mathrm{~d}, \mathrm{~J}=10.2 \mathrm{~Hz}, 2 \mathrm{H}), 5.03(\mathrm{~d}, \mathrm{~J}=$ $17.0 \mathrm{~Hz}, 2 \mathrm{H}), 2.45$ (s, 3H). ${ }^{13} \mathbf{C}-\mathbf{N M R}\left(100 \mathrm{MHz}, \mathrm{CDCl}_{3}, \mathrm{CDCl}_{3}\right.$, diagnostic signals) $\delta 46.3,34.1$.

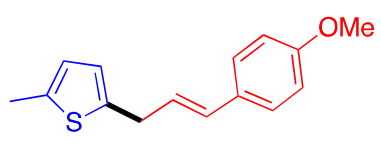

3ad+3ad' (98:2). Colourless oil. Yield $=33 \%(8 \mathrm{mg}),\left(c \mathrm{Hex}: \mathrm{Et}_{2} \mathrm{O}=98: 2\right) .{ }^{\boldsymbol{I}} \boldsymbol{H}-\boldsymbol{N M R}\left(500 \mathrm{MHz}, \mathrm{CDCl}_{3}\right) \delta 7.30(\mathrm{~d}, \mathrm{~J}=$ $9.0 \mathrm{~Hz}, 2 \mathrm{H}), 6.84(\mathrm{~d}, \mathrm{~J}=9.0 \mathrm{~Hz}, 2 \mathrm{H}), 6.62(\mathrm{~d}, \mathrm{~J}=3.0 \mathrm{~Hz}, 1 \mathrm{H}), 6.58(\mathrm{~d}, \mathrm{~J}=3.0 \mathrm{~Hz}, 1 \mathrm{H}), 6.44(\mathrm{~d}, \mathrm{~J}=15.5 \mathrm{~Hz}, 1 \mathrm{H}), 6.20$ 
$(\mathrm{dt}, \mathrm{J}=7.0 \mathrm{~Hz}, \mathrm{~J}=16.0 \mathrm{~Hz}, 1 \mathrm{H}), 3.81(\mathrm{~s}, 3 \mathrm{H}), 3.63(\mathrm{~d}, \mathrm{~J}=6.5 \mathrm{~Hz}, 2 \mathrm{H}), 2.44(\mathrm{~s}, 3 \mathrm{H}) .{ }^{13} \boldsymbol{C}-\boldsymbol{N M R}\left(125 \mathrm{MHz}, \mathrm{CDCl}_{3}\right) \delta$ 159.0, 141.2, 138.1, 130.5, 130.1, 127.3, 126.3, 124.8, 124.3, 113.9, 55.3, 33.5, 15.3. GC-MS (m/z): 145, $229,244$. Anal. Calc. for $\left(\mathrm{C}_{15} \mathrm{H}_{16} \mathrm{OS}\right.$ : 244.09): C, 73.73; H, 6.60; found: C, 73.54, H, 6.31.

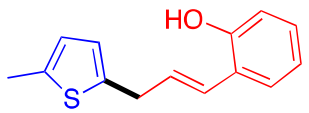

3ae+3ae'+ $\boldsymbol{b}$-3ae (76:15:9). Colourless oil. Yield $=39 \%(9 \mathrm{mg}),\left(c \mathrm{Hex}_{\mathrm{Et}} \mathrm{O}=85: 15\right) .{ }^{\boldsymbol{I}} \boldsymbol{H}-\boldsymbol{N M \boldsymbol { R }}\left(400 \mathrm{MHz}, \mathrm{CDCl}_{3}\right) \delta$ $7.32(\mathrm{dd}, \mathrm{J}=8.0 \mathrm{~Hz}, \mathrm{~J}=2.8 \mathrm{~Hz}, 1 \mathrm{H}), 7.19(\mathrm{dt}, \mathrm{J}=8.0 \mathrm{~Hz}, \mathrm{~J}=1.2 \mathrm{~Hz}, 1 \mathrm{H}), 6.87(\mathrm{t}, \mathrm{J}=7.3 \mathrm{~Hz}, 1 \mathrm{H}), 6.78(\mathrm{~d}, \mathrm{~J}=8.0 \mathrm{~Hz}$, $1 \mathrm{H}), 6.70(\mathrm{~d}, \mathrm{~J}=15.2 \mathrm{~Hz} 1 \mathrm{H}), 6.62(\mathrm{~d}, \mathrm{~J}=4.0 \mathrm{~Hz}, 1 \mathrm{H}), 6.57(\mathrm{~d}, \mathrm{~J}=4.0 \mathrm{~Hz}, 1 \mathrm{H}), 6.34(\mathrm{dt}, \mathrm{J}=7.2 \mathrm{~Hz}, \mathrm{~J}=16.0 \mathrm{~Hz}, 1 \mathrm{H})$, $3.68(\mathrm{~d}, \mathrm{~J}=7.4 \mathrm{~Hz}, 2 \mathrm{H}), 2.45(\mathrm{~s}, 3 \mathrm{H}) .{ }^{13} \boldsymbol{C}-\mathbf{N M R}\left(100 \mathrm{MHz}, \mathrm{CDCl}_{3}\right) \delta 152.6,140.7,130.7,128.3,127.6,125.5,124.8$, 124.6, 124.4, 120.9, 115.7, 33.9, 15.3. GC-MS (m/z): 124, 215, 230. Anal. Calc. for $\left(\mathrm{C}_{14} \mathrm{H}_{14} \mathrm{OS}: 230.08\right): \mathrm{C}, 73.01 ; \mathrm{H}$, 6.13; found: C, 72.88, H, 6.25.

3ae ${ }^{1} \boldsymbol{H}$-NMR (400 MHz, $\mathrm{CDCl}_{3}$, diagnostic signals) $\delta 3.45(\mathrm{~d}, \mathrm{~J}=6.6 \mathrm{~Hz}, 2 \mathrm{H}), 2.41(\mathrm{~s}, 3 \mathrm{H})$.

b-3ae ${ }^{1}$ H-NMR $\left(400 \mathrm{MHz}, \mathrm{CDCl}_{3}\right.$, diagnostic signals) $\delta 5.25(\mathrm{~d}, \mathrm{~J}=9.9 \mathrm{~Hz}, 1 \mathrm{H}), 5.10(\mathrm{~d}, \mathrm{~J}=17.0 \mathrm{~Hz}, 1 \mathrm{H}), 5.02(\mathrm{~d}, \mathrm{~J}=$ $6.8 \mathrm{~Hz}, 1 \mathrm{H}), 2.39(\mathrm{~s}, 3 \mathrm{H})$.

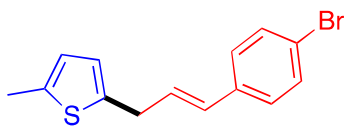

3af+3af' (75:21:4). Colourless oil. Yield $=56 \%(16 \mathrm{mg}),\left(n\right.$-Pent). ${ }^{\mathbf{1}} \boldsymbol{H}-\boldsymbol{N M R}\left(500 \mathrm{MHz}, \mathrm{CDCl}_{3}\right) \delta 7.43-7.40(\mathrm{~m}, 2 \mathrm{H})$, 7.23-7.19 (m, 2H), $6.64(\mathrm{~d}, \mathrm{~J}=12.0 \mathrm{~Hz}, 2 \mathrm{H}), 6.43(\mathrm{~d}, \mathrm{~J}=16.0 \mathrm{~Hz}, 1 \mathrm{H}), 6.37-6.33(\mathrm{~m}, 1 \mathrm{H}), 3.64(\mathrm{~d}, \mathrm{~J}=6.5 \mathrm{~Hz}, 2 \mathrm{H}), 2.45$ (s, 3H). ${ }^{13}$ C-NMR $\left(125 \mathrm{MHz}, \mathrm{CDCl}_{3}\right) \delta 140.3,138.3,136.2,131.6,131.5,129.9,129.3,127.7,127.6,124.9,124.5$, 120.9, 33.5, 15.3. GC-MS (m/z): 292-294. Anal. Calc. for $\left(\mathrm{C}_{14} \mathrm{H}_{14} \mathrm{BrS}: 291.99\right)$ : C, 57.35; H, 4.47; found: C, 57.21, H, 4.23 .

3af' ${ }^{1} \boldsymbol{H}$-NMR $\left(500 \mathrm{MHz}, \mathrm{CDCl}_{3}\right.$, diagnostic signals) $\delta 7.03(\mathrm{~d}, \mathrm{~J}=5.5 \mathrm{~Hz}, 1 \mathrm{H}), 6.84(\mathrm{~d}, \mathrm{~J}=5.5 \mathrm{~Hz}, 1 \mathrm{H}), 3.44(\mathrm{~d}, \mathrm{~J}=5.0$ $\mathrm{Hz}, 2 \mathrm{H}), 2.40$ (s, 3H

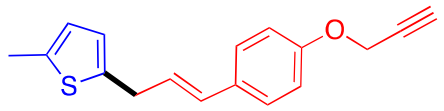

3ag+3ag' (91:9). Pale green oil. Yield = 85\% (23 mg), $\left(c \mathrm{Hex}: \mathrm{Et}_{2} \mathrm{O}=98: 2\right) .{ }^{\boldsymbol{H}} \boldsymbol{H}-\boldsymbol{N M R}\left(400 \mathrm{MHz}, \mathrm{CDCl}_{3}\right) \delta 7.29(\mathrm{~d}, \mathrm{~J}=$ $8.8 \mathrm{~Hz}, 2 \mathrm{H}), 6.89(\mathrm{~d}, \mathrm{~J}=8.8 \mathrm{~Hz}, 2 \mathrm{H}), 6.59(\mathrm{dd}, \mathrm{J}=3.3 \mathrm{~Hz}, \mathrm{~J}=15.7 \mathrm{~Hz}, 1 \mathrm{H}), 6.56(\mathrm{~d}, \mathrm{~J}=4.8 \mathrm{~Hz}, 1 \mathrm{H}), 6.42(\mathrm{~d}, \mathrm{~J}=15.7$ $\mathrm{Hz}, 1 \mathrm{H}), 6.20(\mathrm{dt}, \mathrm{J}=6.8 \mathrm{~Hz}, \mathrm{~J}=15.7 \mathrm{~Hz}, 1 \mathrm{H}), 4.68(\mathrm{~d}, \mathrm{~J}=2.4 \mathrm{~Hz}, 2 \mathrm{H}), 3.63(\mathrm{~d}, \mathrm{~J}=7.2 \mathrm{~Hz}, 2 \mathrm{H}), 2.52(\mathrm{~s}, 1 \mathrm{H}), 2.43(\mathrm{~s}$, 3H). ${ }^{13}$ C-NMR (100 MHz, $\left.\mathrm{CDCl}_{3}\right) \delta$ 156.8, 131.0, 130.4, 127.3(2C), 126.8, 124.8, 124.3, 115.0(2C), 78.5, 75.5, 55.8, 33.5, 29.7, 26.9, 15.3. GC-MS (m/z): 229, 268. Anal. Calc. for $\left(\mathrm{C}_{14} \mathrm{H}_{14} \mathrm{OS}: 268.09\right): \mathrm{C}, 76.08 ; \mathrm{H}, 6.01$; found: C, 75.85, $\mathrm{H}, 5.78$.

3af' ${ }^{1} \boldsymbol{H}$-NMR (400 MHz, $\mathrm{CDCl}_{3}$, diagnostic signals) $\delta 3.41(\mathrm{~d}, \mathrm{~J}=7.2 \mathrm{~Hz}, 2 \mathrm{H})$.

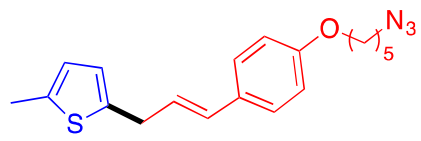

3ah+3ah' (98:2). Yellow oil. Yield = 30\% (10 mg) (cHex:EtOAc: 98:2). ${ }^{\mathbf{H}} \boldsymbol{H}-\boldsymbol{N M R}\left(400 \mathrm{MHz}, \mathrm{CDCl}_{3}\right) \delta 7.28(\mathrm{dd}, \mathrm{J}=$ $1.6 \mathrm{~Hz}, \mathrm{~J}=6.8 \mathrm{~Hz}, 2 \mathrm{H}), 6.81(\mathrm{dd}, \mathrm{J}=1.6 \mathrm{~Hz}, \mathrm{~J}=6.4 \mathrm{~Hz}, 2 \mathrm{H}), 6.59(\mathrm{~d}, \mathrm{~J}=2.8 \mathrm{~Hz}, 1 \mathrm{H}), 6.55(\mathrm{dd}, \mathrm{J}=1.2 \mathrm{~Hz}, \mathrm{~J}=2.8 \mathrm{~Hz}$, $2 \mathrm{H}), 6.40(\mathrm{~d}, \mathrm{~J}=15.6 \mathrm{~Hz}, 1 \mathrm{H}), 6.18(\mathrm{dt}, \mathrm{J}=6.8 \mathrm{~Hz}, \mathrm{~J}=15.6 \mathrm{~Hz}, 1 \mathrm{H}), 3.94(\mathrm{t}, \mathrm{J}=7.2 \mathrm{~Hz}, 2 \mathrm{H}), 3.60(\mathrm{~d}, \mathrm{~J}=7.2 \mathrm{~Hz}, 2 \mathrm{H})$, $3.28(\mathrm{t}, \mathrm{J}=7.2 \mathrm{~Hz}, 2 \mathrm{H}), 2.44(\mathrm{~s}, 3 \mathrm{H}), 1.80-1.76(\mathrm{~m}, 2 \mathrm{H}), 1.68-1.63(\mathrm{~m}, 2 \mathrm{H}), 1.56-1.52(\mathrm{~m}, 2 \mathrm{H}) .{ }^{13} \boldsymbol{C}-\boldsymbol{N M R}(100 \mathrm{MHz}$, $\left.\mathrm{CDCl}_{3}\right) \delta 158.3,141.2,130.5,130.1,127.3(2 \mathrm{C}), 126.2,124.8,124.2,114.5(2 \mathrm{C}), 67.6,51.3,33.5,29.7,28.8,28.6,26.9$, 23.4, 15.3. DEP 313, 341. Anal. Calc. for $\left(\mathrm{C}_{19} \mathrm{H}_{23} \mathrm{~N}_{3} \mathrm{OS}\right.$ : 341.16): C, 66.83; H, 6.79; found: C, 66.95, H, 6.55. 


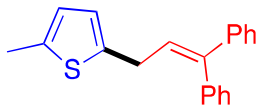

3ai+3ai' (71:29). Colourless oil. Yield = 69\% (20 mg), (cHex). ${ }^{1} \boldsymbol{H}-\mathbf{N M R}\left(500 \mathrm{MHz}, \mathrm{CDCl}_{3}\right) \delta$ 7.44-7.40 (m, 3H), 7.36$7.34(\mathrm{~m}, 1 \mathrm{H}), 7.32-7.26(\mathrm{~m}, 6 \mathrm{H}), 6.61(\mathrm{bs}, 1 \mathrm{H}), 6.59$ (bs, 1H) $6.28(\mathrm{t}, \mathrm{J}=7.6 \mathrm{~Hz}, 1 \mathrm{H}), 3.56(\mathrm{~d}, \mathrm{~J}=7.6 \mathrm{~Hz}, 2 \mathrm{H}), 2.46(\mathrm{~s}$, 3H). ${ }^{13}$ C-NMR $\left(125 \mathrm{MHz}, \mathrm{CDCl}_{3}\right) \delta 142.6,142.3,141.5,139.5,137.9,129.8,128.3,128.1,127.4,127.2,127.1,126.9$, 124.8, 123.9, 30.5, 15.3 GC-MS (m/z): 290. Anal. Calc. for $\left(\mathrm{C}_{20} \mathrm{H}_{18} \mathrm{~S}: 290.11\right)$ : C, 82.71; H, 6.25; found: C, 82.51, H, 6.05 .

3ai' ${ }^{1}$ H-NMR $\left(500 \mathrm{MHz}, \mathrm{CDCl}_{3}\right.$, diagnostic signals) $\delta: 7.02(\mathrm{~d}, \mathrm{~J}=5.2 \mathrm{~Hz}, 1 \mathrm{H}), 6.87(\mathrm{~d}, \mathrm{~J}=5.2 \mathrm{~Hz}, 1 \mathrm{H}), 6.22(\mathrm{t}, \mathrm{J}=7.5$ $\mathrm{Hz}, 1 \mathrm{H}), 3.36(\mathrm{~d}, \mathrm{~J}=7.5 \mathrm{~Hz}, 2 \mathrm{H}), 2.30(\mathrm{~s}, 3 \mathrm{H}) .{ }^{13} \boldsymbol{C}-\mathbf{N M R}\left(125 \mathrm{MHz}, \mathrm{CDCl}_{3}\right.$, diagnostic signals) $\delta:$ 129.9, 128.1, 127.1, 121.0, 28.8, 12.9 .

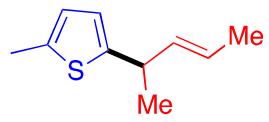

3aj+3aj' (98:2). Colourless oil. Yield = 45\% (8 mg), $(c \mathrm{Hex}) .{ }^{\boldsymbol{I}} \boldsymbol{H}-\mathbf{N M R}\left(500 \mathrm{MHz}, \mathrm{CDCl}_{3}\right) \delta 6.57(\mathrm{bs}, 2 \mathrm{H}), 5.60-5.50$ $(\mathrm{m}, 2 \mathrm{H}), 3.60-3.56(\mathrm{~m}, 1 \mathrm{H}), 2.44(\mathrm{~s}, 3 \mathrm{H}), 1.69(\mathrm{~d}, \mathrm{~J}=5.5 \mathrm{~Hz}, 3 \mathrm{H}), 1.38(\mathrm{~d}, \mathrm{~J}=7.0 \mathrm{~Hz}, 3 \mathrm{H}) .{ }^{13} \boldsymbol{C}-\boldsymbol{N M R}(125 \mathrm{MHz}$, $\left.\mathrm{CDCl}_{3}\right) \delta 148.5,137.3,135.8,124.5,124.1,122.3,38.2,22.3,17.7,15.3$. GC-MS (m/z): 166. Anal. Calc. for $\left(\mathrm{C}_{10} \mathrm{H}_{14} \mathrm{~S}\right.$ : 166.08): C, 72.23; H, 8.49; found: C, 72.39, H, 8.51.

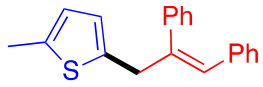

3ak+3ak'+b-3ak (75:18:7). Colourless oil. Yield = 62\% (18 mg) (n-Pent:Et $\left.\mathrm{O}_{2} \mathrm{O}=98: 2\right)$. ${ }^{\boldsymbol{I}} \boldsymbol{H}-\boldsymbol{N M \boldsymbol { R }}\left(500 \mathrm{MHz}, \mathrm{CDCl}_{3}\right) \delta$ $7.33-7.25(\mathrm{~m}, 4 \mathrm{H}), 7.14(\mathrm{~d}, \mathrm{~J}=6.5 \mathrm{~Hz}, 2 \mathrm{H}), 7.10-7.07(\mathrm{~m}, 4 \mathrm{H}), 6.96(\mathrm{~d}, \mathrm{~J}=6.5 \mathrm{~Hz}, 2 \mathrm{H}), 6.56(\mathrm{~s}, 1 \mathrm{H}), 3.90$ (s, $2 \mathrm{H}), 2.42$ (s, 3H). ${ }^{13} \boldsymbol{C}$-NMR $\left(125 \mathrm{MHz}, \mathrm{CDCl}_{3}\right) \delta$ 141.3, 140.6, 139.9, 138.4, 137.1, 129.1, 128.7, 128.4, 128.0, 127.8, 127.1, 126.4, 125.4, 124.7, 41.0, 15.4. GC-MS (m/z): 111, 178, 275, 290. Anal. Calc. for $\left(\mathrm{C}_{20} \mathrm{H}_{18} \mathrm{~S}: 290.11\right)$ : C, 82.71; H, 6.25; found: C, 82.91, H, 6.15.

3ak' ${ }^{1} \boldsymbol{H}$-NMR $\left(500 \mathrm{MHz}, \mathrm{CDCl}_{3}\right.$, diagnostic signals) $\delta: 6.34(\mathrm{~s}, 1 \mathrm{H}), 3.68(\mathrm{~s}, 2 \mathrm{H}), 2.26(\mathrm{~s}, 3 \mathrm{H}) .{ }^{13} \boldsymbol{C}-\boldsymbol{N M R}(125 \mathrm{MHz}$, $\mathrm{CDCl}_{3}$, diagnostic signals) $\delta: 129.6,120.9,39.4$.

b-3ak ${ }^{1} \boldsymbol{H}$-NMR (500 MHz, $\mathrm{CDCl}_{3}$, diagnostic signals) $\delta: 5.66$ (s, 1H), 5.47 (s, 1H), 5.01 (s, 1H). ${ }^{13} \boldsymbol{C}$ - $\mathbf{N M R}(125 \mathrm{MHz}$, $\mathrm{CDCl}_{3}$, diagnostic signals) $\delta: 51.2$.

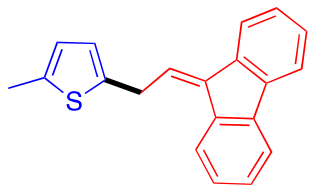

3al+3al' (90:10). Yellow wax. Yield $=56 \%(16 \mathrm{mg}),\left(c \mathrm{Hex}_{2} \mathrm{Et}_{2} \mathrm{O}=95: 5\right) .{ }^{1} \boldsymbol{H}-\boldsymbol{N M R}\left(400 \mathrm{MHz}, \mathrm{CDCl}_{3}\right) \delta 7.87(\mathrm{~d}, \mathrm{~J}=$ $7.6 \mathrm{~Hz}, 1 \mathrm{H}), 7.77(\mathrm{~d}, \mathrm{~J}=7.4 \mathrm{~Hz}, 1 \mathrm{H}), 7.65(\mathrm{~d}, \mathrm{~J}=7.6 \mathrm{~Hz}, 1 \mathrm{H}), 7.62(\mathrm{~d}, \mathrm{~J}=7.4 \mathrm{~Hz}, 1 \mathrm{H}), 7.42-7.24(\mathrm{~m}, 4 \mathrm{H}), 6.85(\mathrm{t}, J=$ $7.4 \mathrm{~Hz}, 1 \mathrm{H}), 6.72(\mathrm{~d}, J=3.4 \mathrm{~Hz}, 1 \mathrm{H}), 6.60(\mathrm{dd}, \mathrm{J}=1.3 \mathrm{~Hz}, \mathrm{~J}=3.5 \mathrm{~Hz}, 1 \mathrm{H}), 4.29(\mathrm{~d}, \mathrm{~J}=7.5 \mathrm{~Hz}, 2 \mathrm{H}), 2.44(\mathrm{~s}, 3 \mathrm{H}) .{ }^{13} \boldsymbol{C}$ NMR $\left(100 \mathrm{MHz}, \mathrm{CDCl}_{3}\right) \delta 141.1,139.7,139.2,138.8,138.5,137.1,136.1,138.1,127.8,127.6,127.1,127.0,125.0$, 124.9, 124.8, 120.9, 119.9, 119.5, 30.0, 15.3. GC-MS (m/z): 273, 288. Anal. Calc. for $\left(\mathrm{C}_{20} \mathrm{H}_{16} \mathrm{~S}: 288.10\right): \mathrm{C}, 83.29 ; \mathrm{H}$, 5.59; found: C, 83.05, H, 5.41.

3al' ${ }^{1} \boldsymbol{H}$-NMR $\left(400 \mathrm{MHz}, \mathrm{CDCl}_{3}\right.$, diagnostic signals) $\delta 7.92(\mathrm{~d}, \mathrm{~J}=7.6 \mathrm{~Hz}, 1 \mathrm{H}), 7.05(\mathrm{~d}, \mathrm{~J}=6.0 \mathrm{~Hz}, 1 \mathrm{H}), 6.92(\mathrm{~d}, \mathrm{~J}=$ $6.0 \mathrm{~Hz}, 1 \mathrm{H}), 4.09(\mathrm{~d}, \mathrm{~J}=7.1 \mathrm{~Hz}, 1 \mathrm{H}), 2.46(\mathrm{~s}, 3 \mathrm{H}) .{ }^{13} \boldsymbol{C}$-NMR $\left(100 \mathrm{MHz}, \mathrm{CDCl}_{3}\right.$, diagnostic signals $) \delta: 29,7$. 


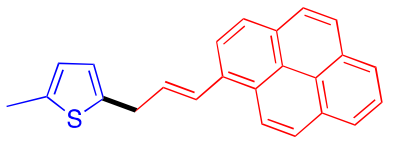

3am+3am' (90:10). Pale yellow wax. Yield $=70 \%(25 \mathrm{mg}),\left(n \mathrm{Pent}_{\mathrm{Et}} \mathrm{O}=97: 3\right) .{ }^{\mathbf{H}} \boldsymbol{H}-\boldsymbol{N M \boldsymbol { R }}\left(400 \mathrm{MHz}, \mathrm{CDCl}_{3}\right) \delta: 8.38$ $(\mathrm{d}, \mathrm{J}=9.2 \mathrm{~Hz}, 1 \mathrm{H}), 8.16-8.07(\mathrm{~m}, 5 \mathrm{H}), 8.02(\mathrm{~s}, 2 \mathrm{H}), 7.97(\mathrm{t}, \mathrm{J}=7.8 \mathrm{~Hz}, 1 \mathrm{H}), 7.52(\mathrm{~d}, \mathrm{~J}=16.0 \mathrm{~Hz}, 1 \mathrm{H}), 6.72(\mathrm{bs}, 1 \mathrm{H})$, 6.62 (bs, 1H), 6.59-6.57 (m, 1H), $3.87(\mathrm{~d}, \mathrm{~J}=6.4 \mathrm{~Hz}, 2 \mathrm{H}), 2.48(\mathrm{~s}, 3 \mathrm{H}) .{ }^{13} \boldsymbol{C}-\boldsymbol{N M R}\left(100 \mathrm{MHz}, \mathrm{CDCl}_{3}\right) \delta: 140.7,138.4$, 132.1, 132.0, 131.5, 131.0, 130.6, 128.5, 127.4 (2C), 127.4, 125.9, 125.1, 125.0, 124.9 (2C), 124.9, 124.6, 124.1, 123.2, 34.2, 15.4. $\boldsymbol{G C}-\boldsymbol{M S}(\mathrm{m} / \mathrm{z}): 239,338$. Anal. Calc. for $\left(\mathrm{C}_{25} \mathrm{H}_{20} \mathrm{~S}: 352.13\right)$ : C, 85.19; H, 5.72; found: C, 84.98, H, 5.65.

3am' ${ }^{1} \boldsymbol{H}$-NMR $\left(400 \mathrm{MHz}, \mathrm{CDCl}_{3}\right.$, diagnostic signals) $\delta 8.31(\mathrm{~d}, \mathrm{~J}=8.0 \mathrm{~Hz}, 1 \mathrm{H}), 7.41(\mathrm{~d}, \mathrm{~J}=16.0 \mathrm{~Hz}, 1 \mathrm{H}), 7.07(\mathrm{~d}, \mathrm{~J}=$ $6.0 \mathrm{~Hz}, 1 \mathrm{H}), 6.96(\mathrm{~d}, \mathrm{~J}=8.0 \mathrm{~Hz}, 1 \mathrm{H}), 3.64(\mathrm{~d}, \mathrm{~J}=8.0 \mathrm{~Hz}, 1 \mathrm{H}), 2.48$ (s, 3H). ${ }^{13} \boldsymbol{C}$-NMR $\left(100 \mathrm{MHz}, \mathrm{CDCl}_{3}\right.$, diagnostic signals) $\delta: 36.5$.

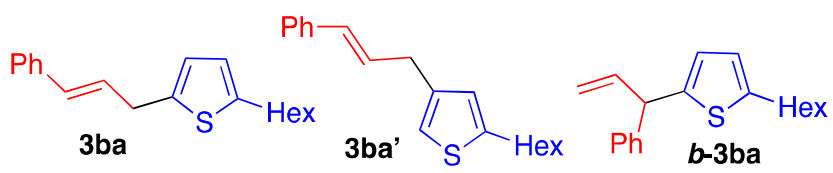

3ba:3ba': $\boldsymbol{b}$-3ba $(77: 16: 7)$. Colourless oil $\left(\mathrm{GO}=25 \mathrm{wt} \%, 90 \mathrm{C}^{\circ}, 6 \mathrm{~h}\right)$. Yield $=51 \%(8 \mathrm{mg})(\mathrm{cHex}) .{ }^{\mathbf{l}} \boldsymbol{H}-\mathbf{N M R}(500 \mathrm{MHz}$, $\left.\mathrm{CDCl}_{3}\right) \delta 7.39-7.37(\mathrm{~m}, 2 \mathrm{H}), 7.35-7.28(\mathrm{~m}, 2 \mathrm{H}), 7.24-7.20(\mathrm{~m}, 1 \mathrm{H}), 6.65(\mathrm{bs}, 1 \mathrm{H}), 6.61(\mathrm{bs}, 1 \mathrm{H}), 6.50(\mathrm{~d}, \mathrm{~J}=16.0 \mathrm{~Hz}$, $1 \mathrm{H}), 6.39-6.31(\mathrm{~m}, 1 \mathrm{H}), 3.67(\mathrm{~d}, \mathrm{~J}=7.0 \mathrm{~Hz}, 2 \mathrm{H}), 2.76(\mathrm{t}, \mathrm{J}=8.0 \mathrm{~Hz}, 2 \mathrm{H}), 1.67-1.62(\mathrm{~m}, 2 \mathrm{H}), 1.40-1.27(\mathrm{~m}, 6 \mathrm{H}), 0.91-$ 0.89 (m, 3H). ${ }^{13}$ C-NMR (125 MHz, $\left.\mathrm{CDCl}_{3}\right) \delta$ 144.4, 140.4, 137.3, 131.1, 128.5, 128.4, 127.2, 126.2, 124.1, 123.6, 33.6, 31.7, 31.6, 30.2, 28.8, 22.6, 14.1. GC-MS (m/z): 284. Anal. Calc. for $\left(\mathrm{C}_{19} \mathrm{H}_{22} \mathrm{OS}: 298.14\right)$ : C, 76.47; H, 7.43; found: C, $76.32, \mathrm{H}, 7.22$.

3ba' ${ }^{1} \boldsymbol{H}$-NMR $\left(500 \mathrm{MHz}, \mathrm{CDCl}_{3}\right.$, diagnostic signals) $\delta 7.06(\mathrm{~d}, \mathrm{~J}=5.0 \mathrm{~Hz}, 1 \mathrm{H}), 6.86(\mathrm{~d}, \mathrm{~J}=5.0 \mathrm{~Hz}, 1 \mathrm{H}), 3.46(\mathrm{~d}, \mathrm{~J}=$ $6.5 \mathrm{~Hz}, 2 \mathrm{H})$.

b-3ba ${ }^{1} \boldsymbol{H}$-NMR $\left(500 \mathrm{MHz}, \mathrm{CDCl}_{3}\right.$, diagnostic signals) $\delta 5.20(\mathrm{~d}, \mathrm{~J}=10.5 \mathrm{~Hz}, 1 \mathrm{H}), 5.10(\mathrm{~d}, \mathrm{~J}=17.5 \mathrm{~Hz}, 1 \mathrm{H}), 4.83(\mathrm{~d}, \mathrm{~J}$ $=7.5 \mathrm{~Hz}, 1 \mathrm{H})$.

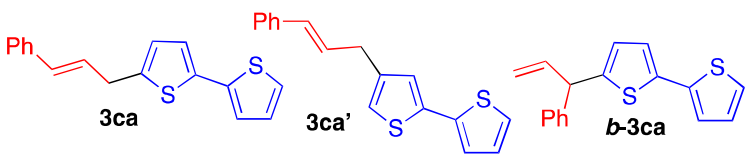

3ca+3ca'+b-3ca (60:33:7). Yellow oil $\left(\mathrm{GO}=50 \mathrm{wt} \%, 90 \mathrm{C}^{\circ}, 6 \mathrm{~h}\right)$. Yield $=48 \%(14 \mathrm{mg})(n \mathrm{Hex}) .{ }^{\mathbf{I}} \boldsymbol{H}-\mathbf{N M R}(400 \mathrm{MHz}$, $\left.\mathrm{CDCl}_{3}\right) \delta: 7.40(\mathrm{~d}, \mathrm{~J}=6.8 \mathrm{~Hz}, 1 \mathrm{H}), 7.37-7.30(\mathrm{~m}, 3 \mathrm{H}), 7.26-7.22(\mathrm{~m}, 1 \mathrm{H}), 7.20-7.16(\mathrm{~m}, 1 \mathrm{H}), 7.12-7.07(\mathrm{~m}, 1 \mathrm{H}), 7.04-$ $7.00(\mathrm{~m}, 2 \mathrm{H}), 6.78(\mathrm{~d}, \mathrm{~J}=3.3 \mathrm{~Hz}, 1 \mathrm{H}), 6.55(\mathrm{~d}, \mathrm{~J}=15.6 \mathrm{~Hz}, 1 \mathrm{H}), 6.42-6.38(\mathrm{~m}, 1 \mathrm{H}), 3.72(\mathrm{~d}, \mathrm{~J}=6.8 \mathrm{~Hz}, 2 \mathrm{H}) .{ }^{13} \boldsymbol{C}-\boldsymbol{N M \boldsymbol { R }}$ $\left(100 \mathrm{MHz}, \mathrm{CDCl}_{3}\right) \delta 142.5,137.1,135.8,131.7,130.2,128.5,128.5,127.7,127.4,126.2,126.1,125.4,123.6,123.2$, 33.5. GC-MS (m/z): 115, 179, 282. Anal. Calc. for $\left(\mathrm{C}_{17} \mathrm{H}_{14} \mathrm{~S}_{2}: 282.05\right)$ : C, 72.30; H, 5.00; found: C, 72.45, H, 5.12.

3da' ${ }^{1} \boldsymbol{H}$-NMR $\left(400 \mathrm{MHz}, \mathrm{CDCl}_{3}\right.$, diagnostic signals) $\delta 3.68(\mathrm{~d}, \mathrm{~J}=5.0 \mathrm{~Hz}, 2 \mathrm{H}) .{ }^{13} \boldsymbol{C}-\mathbf{N M R}\left(100 \mathrm{MHz}, \mathrm{CDCl}_{3}\right.$, diagnostic signals) $\delta: 30.3$.

b-3da ${ }^{1} \boldsymbol{H}$-NMR $\left(400 \mathrm{MHz}, \mathrm{CDCl}_{3}\right.$, diagnostic signals $) \delta 5.26(\mathrm{~d}, \mathrm{~J}=9.6 \mathrm{~Hz}, 1 \mathrm{H}), 5.16(\mathrm{~d}, \mathrm{~J}=17.2 \mathrm{~Hz}, 1 \mathrm{H}), 4.88(\mathrm{~d}, \mathrm{~J}=$ $7.2 \mathrm{~Hz}, 1 \mathrm{H})$.

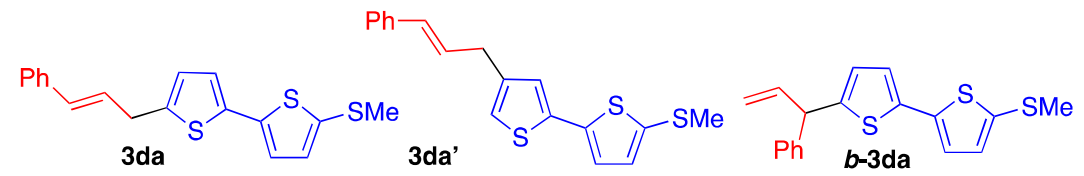

3da+3da'+b-3da+3da'" $(68: 19: 13)$. Yellow oil $\left(\mathrm{GO}=50 \mathrm{wt} \%, 90 \mathrm{C}^{\circ}, 6 \mathrm{~h}\right)$. Yield $=27 \%(9 \mathrm{mg}),(\mathrm{cHex}) .{ }^{\mathbf{H}} \boldsymbol{H}-\mathbf{N M R}(500$ $\left.\mathrm{MHz} \mathrm{CDCl}_{3}\right) \delta$ 7.39-7.34 (m, 2H), 7.33-7.28 (m, 2H) 7.25 -7.20 (m, 2H), 7.15-7.09 (m, 1H), 7.02-6.95 (m, $\left.2 \mathrm{H}\right), 6.47$ $(\mathrm{d}, \mathrm{J}=16.0 \mathrm{~Hz}, 1 \mathrm{H}), 6.39-6.29(\mathrm{~m}, 1 \mathrm{H}), 3.60(\mathrm{~d}, \mathrm{~J}=7.0 \mathrm{~Hz}, 2 \mathrm{H}), 2.43(\mathrm{~s}, 3 \mathrm{H}) .{ }^{13} \boldsymbol{C}-\boldsymbol{N M R}\left(125 \mathrm{MHz}, \mathrm{CDCl}_{3}\right) \delta 144.3$, 
138.5, 137.3, 131.1, 130.3, 128.5, 128.1, 127.8, 127.2, 126.1, 125.4, 124.6, 123.8, 32.6, 22.2. GC-MS (m/z): 328. Anal. Calc. for $\left(\mathrm{C}_{18} \mathrm{H}_{16} \mathrm{~S}_{3}\right.$ : 328.04): C, 65.81; H, 4.91; found: C, 65.61, H, 4.66.

3da ${ }^{1} \boldsymbol{H}$-NMR $\left(500 \mathrm{MHz}, \mathrm{CDCl}_{3}\right.$, diagnostic signals) $\delta 6.93(\mathrm{~d}, \mathrm{~J}=3.0 \mathrm{~Hz}, 1 \mathrm{H}), 6.76(\mathrm{~d}, \mathrm{~J}=3.0 \mathrm{~Hz}, 1 \mathrm{H}), 6.53(\mathrm{~d}, \mathrm{~J}=$ $16.0 \mathrm{~Hz}, 1 \mathrm{H}), 3.79(\mathrm{~d}, \mathrm{~J}=7.0 \mathrm{~Hz}, 2 \mathrm{H}), 2.50$ (s, 3H).

b-3da ${ }^{1} \boldsymbol{H}$-NMR $\left(500 \mathrm{MHz}, \mathrm{CDCl}_{3}\right.$, diagnostic signals $): 3.66(\mathrm{~d}, \mathrm{~J}=5.5 \mathrm{~Hz}, 2 \mathrm{H}), 2.52(\mathrm{~s}, 3 \mathrm{H})$.

Traces of a forth undefined isomer 3da" were also detected by NMR. ${ }^{\mathbf{I}} \boldsymbol{H}$-NMR (500 MHz, CDCl ${ }_{3}$, diagnostic signals): $3.68(\mathrm{~d}, \mathrm{~J}=6.5 \mathrm{~Hz}, 2 \mathrm{H}), 2.51(\mathrm{~s}, 3 \mathrm{H})$.

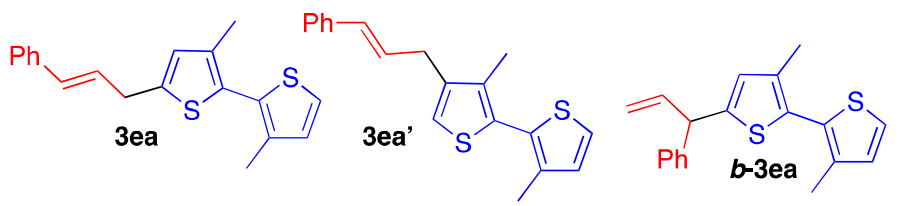

3ea+3ea'+b-3ea (63:18:19). Yellow oil $\left(\mathrm{GO}=50 \mathrm{wt} \%, 90 \mathrm{C}^{\circ}, 6 \mathrm{~h}\right)$. Yield $=60 \%(19 \mathrm{mg}),\left(n \mathrm{Hex}: \mathrm{Et}_{2} \mathrm{O}=96: 4\right) .{ }^{\boldsymbol{I}} \boldsymbol{H}$ NMR $\left(500 \mathrm{MHz}, \mathrm{CDCl}_{3}\right) \delta$ 7.41-7.39 (m, 2H), 7.35-7.30 (m, 3H), 7.26-7.22 (m, 2H), 6.91 (d, J = 5.0 Hz, 1H), 6.54 (d, J $=16.0 \mathrm{~Hz}, 1 \mathrm{H}), 6.41-6.35(\mathrm{~m}, 1 \mathrm{H}), 3.68(\mathrm{~d}, \mathrm{~J}=7.0 \mathrm{~Hz}, 2 \mathrm{H}), 2.19(\mathrm{~s}, 3 \mathrm{H}), 2.12(\mathrm{~s}, 3 \mathrm{H}) .{ }^{13} \boldsymbol{C}-\boldsymbol{N M R}\left(125 \mathrm{MHz} \mathrm{CDCl}_{3}\right) \delta$ 142.6, 139.9, 137.2, 136.4, 136.2, 131.6, 129.9, 128.5, 128.2, 127.8, 127.8, 127.3, 126.3, 124.7, 33.5, 14.8, 14.7. GCMS (m/z): 310. Anal. Calc. for $\left(\mathrm{C}_{19} \mathrm{H}_{18} \mathrm{~S}_{2}: 310.08\right)$ : C, 73.50; H, 5.84; found: C, 73.66, H, 5.68.

3ea ${ }^{1}$ H-NMR $\left(500 \mathrm{MHz}, \mathrm{CDCl}_{3}\right.$, diagnostic signals) $\delta 6.32-6.29(\mathrm{~m}, 1 \mathrm{H}), 3.48(\mathrm{~d}, \mathrm{~J}=6.0 \mathrm{~Hz}, 2 \mathrm{H})$.

b-3ea ${ }^{1} \boldsymbol{H}$-NMR $\left(500 \mathrm{MHz}, \mathrm{CDCl}_{3}\right.$, diagnostic signals $) \delta 5.23(\mathrm{~d}, \mathrm{~J}=9.5 \mathrm{~Hz}, 1 \mathrm{H}), 5.16(\mathrm{~d}, \mathrm{~J}=17.0 \mathrm{~Hz}, 1 \mathrm{H}), 4.84(\mathrm{~d}, \mathrm{~J}=$ $7.0 \mathrm{~Hz}, 1 \mathrm{H}) .{ }^{13} \mathrm{C}$-NMR $\left(125 \mathrm{MHz}, \mathrm{CDCl}_{3}\right.$, diagnostic signals) $\delta 51.1$.

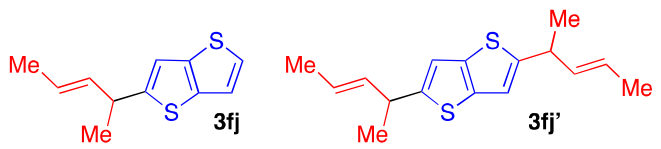

3fj+3fj' (95:5). Colourless oil $\left(\mathrm{GO}=50 \mathrm{wt} \%, 90 \mathrm{C}^{\circ}, 6 \mathrm{~h}\right)$. Yield $=64 \%(13 \mathrm{mg})\left(n\right.$-Pent:EtOAc: $\left.\mathrm{CH}_{2} \mathrm{Cl}_{2}=98: 1: 1\right)$. ${ }^{1} \boldsymbol{H}$ $\operatorname{NMR}\left(500 \mathrm{MHz}, \mathrm{CDCl}_{3}\right) \delta 7.27(\mathrm{~d}, \mathrm{~J}=5.5 \mathrm{~Hz}, 1 \mathrm{H}), 7.18(\mathrm{~d}, \mathrm{~J}=5.5 \mathrm{~Hz}, 1 \mathrm{H}), 6.99(\mathrm{~s}, 1 \mathrm{H}), 5.66-5.56(\mathrm{~m}, 2 \mathrm{H}), 3.73-3.70$ $(\mathrm{m}, 1 \mathrm{H}), 1.71(\mathrm{~d}, \mathrm{~J}=6.0 \mathrm{~Hz}, 3 \mathrm{H}), 1.45(\mathrm{~d}, \mathrm{~J}=6.5 \mathrm{~Hz}, 3 \mathrm{H}) .{ }^{13} \boldsymbol{C}-\mathbf{N M R}\left(125 \mathrm{MHz}, \mathrm{CDCl}_{3}\right) \delta$ 138.7, 137.4, 135.3, 135.1, 125.4, 125.0, 119.5, 115.0, 39.1, 22.1, 17.8. GC-MS (m/z): 193, 208. Anal. Calc. for $\left(\mathrm{C}_{11} \mathrm{H}_{12} \mathrm{~S}_{2}: 208.04\right): \mathrm{C}, 63.42 ; \mathrm{H}$, 5.81; found: C, 63.31, H, 5.69.

3aq' ${ }^{1} \boldsymbol{H}$-NMR (500 MHz, $\mathrm{CDCl}_{3}$, diagnostic signals) $\delta: 3.51-3.47$ (m, 1H). ${ }^{13} \boldsymbol{C}$-NMR (125 $\mathrm{MHz} \mathrm{CDCl}_{3}$, diagnostic signals) $\delta: 39.0,22.2$.

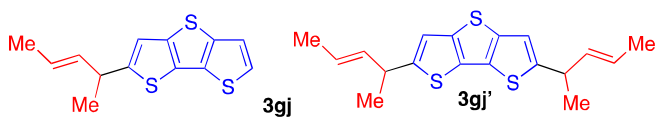

3gj+3gj' (95:5). Colourless oil $\left(\mathrm{GO}=50 \mathrm{wt} \%, 90 \mathrm{C}^{\circ}, 6 \mathrm{~h}\right)$. Yield $=32 \%(8 \mathrm{mg})\left(\mathrm{cHex}: \mathrm{Et}_{2} \mathrm{O}=96: 4\right) .{ }^{\mathbf{I}} \boldsymbol{H}-\boldsymbol{N M R}(400$ $\mathrm{MHz}_{\mathrm{CDCl}}$ ) $\mathrm{C} 7.29(\mathrm{~d}, \mathrm{~J}=5.2 \mathrm{~Hz}, 1 \mathrm{H}), 7.26(\mathrm{~d}, \mathrm{~J}=5.2 \mathrm{~Hz}, 1 \mathrm{H}), 7.00(\mathrm{~s}, 1 \mathrm{H}), 5.67-5.56(\mathrm{~m}, 2 \mathrm{H}), 3.76-3.70(\mathrm{~m}, 1 \mathrm{H})$,

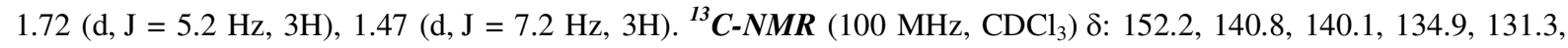
129.0, 125.2, 125.0, 120.7, 116.4, 39.1, 22.1, 17.8. GC-MS (m/z): 249, 264. Anal. Calc. for $\left(\mathrm{C}_{13} \mathrm{H}_{12} \mathrm{~S}_{3}: 264.01\right): \mathrm{C}$ 59.05; H, 4.57; found: C, 59.26, H, 4.69 . 

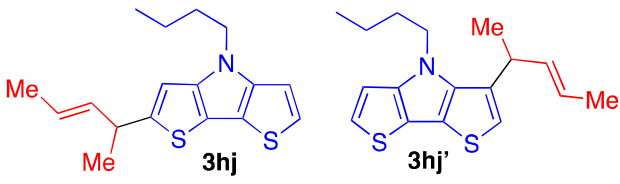

3hj+3hj' (94:6). Colourless oil (GO = 50 wt $\left.\%, 90 \mathrm{C}^{\circ}, 6 \mathrm{~h}\right)$. Yield $=28 \%(9 \mathrm{mg}) .\left(n-\mathrm{Pent}_{\mathrm{Ct}} \mathrm{O}=95: 5\right) .{ }^{\mathbf{H}} \mathbf{H}-\mathbf{N M R}(400$ $\left.\mathrm{MHz}, \mathrm{CDCl}_{3}\right) \delta 7.05(\mathrm{~d}, \mathrm{~J}=5.6 \mathrm{~Hz}, 1 \mathrm{H}), 6.97(\mathrm{~d}, \mathrm{~J}=5.6 \mathrm{~Hz}, 1 \mathrm{H}), 6.73(\mathrm{~s}, 1 \mathrm{H}), 5.68-5.54(\mathrm{~m}, 2 \mathrm{H}), 4.15(\mathrm{t}, \mathrm{J}=7.2 \mathrm{~Hz}$, $2 \mathrm{H}), 3.72-3.68(\mathrm{~m}, 1 \mathrm{H}), 1.87-1.80(\mathrm{~m}, 2 \mathrm{H}), 1.72(\mathrm{~d}, \mathrm{~J}=5.6 \mathrm{~Hz}, 3 \mathrm{H}), 1.46(\mathrm{~d}, \mathrm{~J}=7.2 \mathrm{~Hz}, 3 \mathrm{H}), 1.39-1.33(\mathrm{~m}, 2 \mathrm{H}), 0.94(\mathrm{t}$, $\mathrm{J}=7.6 \mathrm{~Hz}, 3 \mathrm{H}) .{ }^{13} \boldsymbol{C}-\mathbf{N M R}\left(100 \mathrm{MHz}, \mathrm{CDCl}_{3}\right) \delta$ 149.0, 144.0, 143.6, 135.4, 124.6, 121.7, 114.9, 112.5, 110.8, 107.1, 47.0, 39.3, 32.5, 22.2, 20.2, 17.8, 13.7. GC-MS (m/z): 255, 288, 303. Anal. Calc. for $\left(\mathrm{C}_{17} \mathrm{H}_{21} \mathrm{NS}_{2}\right.$ : 303.11): C, 67.28; $\mathrm{H}$, 6.98; found: C, 67.05, H, 6.77 .

3hj' ${ }^{\text {I }} \mathbf{H}$-NMR $\left(400 \mathrm{MHz}, \mathrm{CDCl}_{3}\right)$ 8: $7.12(\mathrm{~d}, \mathrm{~J}=5.2 \mathrm{~Hz}, 1 \mathrm{H}), 7.01(\mathrm{~d}, \mathrm{~J}=5.2 \mathrm{~Hz}, 1 \mathrm{H}), 6.69(\mathrm{~s}, 1 \mathrm{H})$.

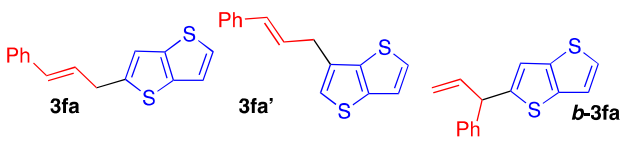

3fa+3fa' $+\boldsymbol{b}$-3fa (85:8:7). White solid. $\left(\mathrm{GO}=50 \mathrm{wt} \%, 90 \mathrm{C}^{\circ}, 6 \mathrm{~h}\right)$. Yield $=50 \%(13 \mathrm{mg}),\left(n\right.$ Pent: $\left.\mathrm{CH}_{2} \mathrm{Cl}_{2}=97: 3\right) . \mathbf{M P}=$ 90-100 ${ }^{\circ} \mathrm{C} .{ }^{1} \boldsymbol{H}-\boldsymbol{N M R}\left(500 \mathrm{MHz}, \mathrm{CDCl}_{3}\right) \delta: 7.39(\mathrm{~d}, \mathrm{~J}=7.5 \mathrm{~Hz}, 2 \mathrm{H}), 7.32(\mathrm{~d}, \mathrm{~J}=7.0 \mathrm{~Hz}, 2 \mathrm{H}), 7.30(\mathrm{~d}, \mathrm{~J}=6.0 \mathrm{~Hz}, 1 \mathrm{H})$, 7.26-7.22 (m, 1H), $7.20(\mathrm{~d}, \mathrm{~J}=5.5 \mathrm{~Hz}, 1 \mathrm{H}), 7.05(\mathrm{~s}, 1 \mathrm{H}), 6.56(\mathrm{~d}, \mathrm{~J}=15.5 \mathrm{~Hz}, 1 \mathrm{H}), 6.40(\mathrm{dt}, \mathrm{J}=6.5 \mathrm{~Hz}, \mathrm{~J}=16.5 \mathrm{~Hz}$, $1 \mathrm{H}), 3.80(\mathrm{~d}, \mathrm{~J}=6.5 \mathrm{~Hz}, 2 \mathrm{H}) .{ }^{13} \boldsymbol{C}-\boldsymbol{N M R}\left(125 \mathrm{MHz}, \mathrm{CDCl}_{3}\right) \delta: 145.7,138.9,138.0,137.1,131.9,128.6,127.5,127.4$, 126.3, 125.7, 119.5, 117.0, 34.6. GC-MS (m/z): 153, 179, 256. Anal. Calc. for $\left(\mathrm{C}_{15} \mathrm{H}_{12} \mathrm{~S}_{2}: 256.04\right): \mathrm{C}, 70.27$; H, 4.72; found: C, 70.45, H, 4.51 .

3fa' ${ }^{\prime} \mathbf{H}-\mathbf{N M R}\left(500 \mathrm{MHz}, \mathrm{CDCl}_{3}\right.$, diagnostic signals) $\delta 6.98(\mathrm{~d}, \mathrm{~J}=13.5 \mathrm{~Hz}, 1 \mathrm{H}), 6.62(\mathrm{~d}, \mathrm{~J}=16.5 \mathrm{~Hz}, 1 \mathrm{H}), 3.66(\mathrm{~d}, \mathrm{~J}=$ $6.5 \mathrm{~Hz}, 2 \mathrm{H}) .{ }^{13} \boldsymbol{C}$-NMR $\left(125 \mathrm{MHz}, \mathrm{CDCl}_{3}\right.$, diagnostic signals) $\delta: 33.6$.

$\boldsymbol{b}$-3fa ${ }^{1} \boldsymbol{H}$-NMR $\left(500 \mathrm{MHz}, \mathrm{CDCl}_{3}\right.$, diagnostic signals) $\delta 6.36-6.29(\mathrm{~m}, 1 \mathrm{H}), 5.27(\mathrm{~d}, \mathrm{~J}=10.0 \mathrm{~Hz}, 1 \mathrm{H}), 5.18(\mathrm{~d}, \mathrm{~J}=17.0$ $\mathrm{Hz}, 1 \mathrm{H}), 4.96(\mathrm{~d}, \mathrm{~J}=7.5 \mathrm{~Hz}, 1 \mathrm{H}) .{ }^{13} \boldsymbol{C}-\boldsymbol{N M R}\left(125 \mathrm{MHz}, \mathrm{CDCl}_{3}\right)$ : 51.6 .

Traces of a forth undefined isomer $3 \mathbf{f a}$ " were also detected by NMR. ${ }^{\mathbf{H}} \mathbf{H}-\mathbf{N M R}\left(500 \mathrm{MHz}, \mathrm{CDCl}_{3}\right.$, diagnostic signals) $\delta 3.55(\mathrm{~d}, \mathrm{~J}=7.0 \mathrm{~Hz}, 2 \mathrm{H})$.

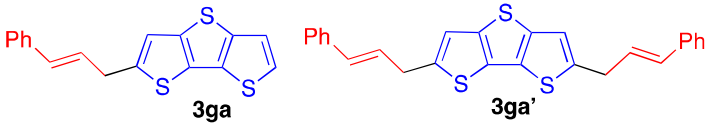

3ga+3ga' (95:5) White solid. (GO $\left.=50 \mathrm{wt} \%, 90 \mathrm{C}^{\circ}, 6 \mathrm{~h}\right)$. Yield $=32 \%(10 \mathrm{mg}),\left(n \mathrm{Pent}: \mathrm{CH}_{2} \mathrm{Cl}_{2}=98: 2\right) . \mathbf{M P}=78-110$ ${ }^{\circ} \mathrm{C} .{ }^{I} \mathrm{H}-\mathrm{NMR}\left(500 \mathrm{MHz}, \mathrm{CDCl}_{3}\right) \delta 7.39(\mathrm{~d}, \mathrm{~J}=6.0 \mathrm{~Hz}, 2 \mathrm{H}), 7.34-7.31(\mathrm{~m}, 3 \mathrm{H}), 7.27-7.23(\mathrm{~m}, 2 \mathrm{H}), 7.07(\mathrm{~s}, 1 \mathrm{H}), 6.57(\mathrm{~d}$, $\mathrm{J}=16.0 \mathrm{~Hz}, 1 \mathrm{H}), 6.41(\mathrm{dt}, \mathrm{J}=7.0 \mathrm{~Hz}, \mathrm{~J}=16.0 \mathrm{~Hz}, 1 \mathrm{H}), 3.82(\mathrm{~d}, \mathrm{~J}=7.0 \mathrm{~Hz}, 2 \mathrm{H}) .{ }^{13} \boldsymbol{C}-\mathbf{N M R}\left(125 \mathrm{MHz}, \mathrm{CDCl}_{3}\right) \delta: 144.4$, 140.9, 140.3, 137.0, 132.1, 131.2, 129.4, 128.6, 127.5, 127.3, 126.3, 125.2, 120.7, 118.3, 34.5. GC-MS (m/z): 209, 235, 312. Anal. Calc. for $\left(\mathrm{C}_{17} \mathrm{H}_{12} \mathrm{~S}_{3}\right.$ : 312.01): C, 65.35; H, 3.87; found: C, 65.12, H, 3.71 .

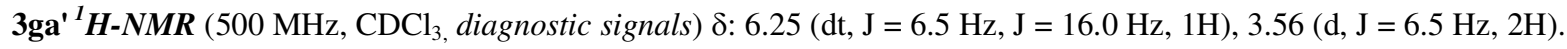

$\boldsymbol{b}$-3ga ${ }^{I} \boldsymbol{H}$-NMR $\left(500 \mathrm{MHz}, \mathrm{CDCl}_{3}\right.$, diagnostic signals) $\delta: 6.35-6.30(\mathrm{~m}, 1 \mathrm{H}), 5.29(\mathrm{~d}, \mathrm{~J}=6.5 \mathrm{~Hz}, 1 \mathrm{H}), 5.19(\mathrm{~d}, \mathrm{~J}=16.5$ $\mathrm{Hz}, 1 \mathrm{H}), 4.98(\mathrm{~d}, \mathrm{~J}=8.0 \mathrm{~Hz}, 1 \mathrm{H})$. 
Figure S1

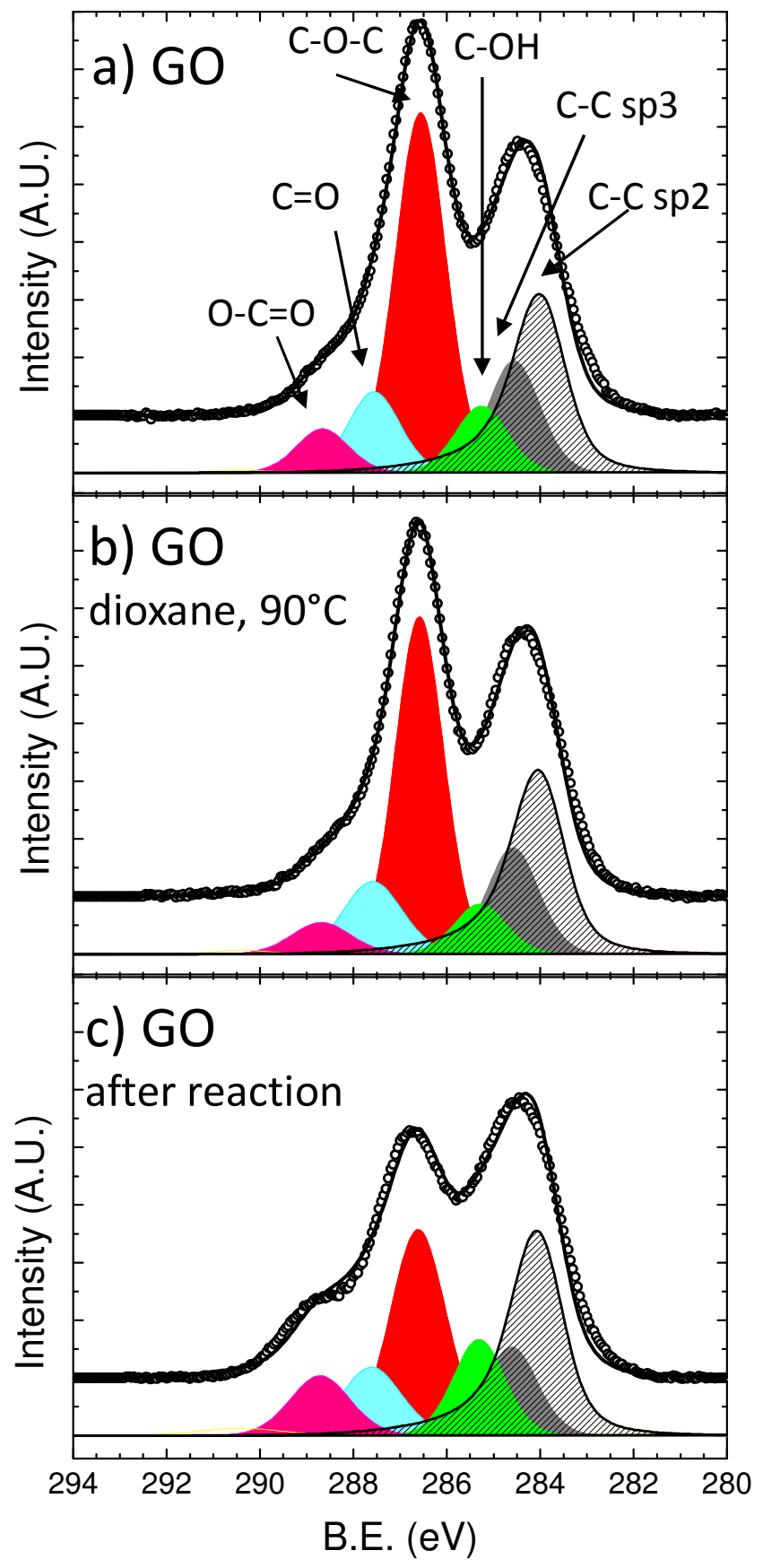

\begin{tabular}{|l|l|l|l|l|l|}
\hline C=C aromatic & C-C defects & C-OH & C-O-C & C=O & O-C=O \\
\hline 284,4 & 285,0 & 285,7 & 286,7 & 288,0 & 290,1 \\
\hline
\end{tabular}


Table S1

\begin{tabular}{llll}
\hline & GO & $\mathbf{G O}^{\circ} \mathbf{9 0}^{\circ} \mathbf{C}$ dioxane & GO after reaction \\
\hline $\mathbf{O} / \mathbf{C}$ & $0.47 \pm 0.03$ & $0.44 \pm 0,03$ & $0.53 \pm 0.03$ \\
\hline $\mathbf{C}-\mathbf{C}(\mathbf{s p 2})$ & $25.1 \pm 0.5$ & $27.7 \pm 0.5$ & $31.4 \pm 0.6$ \\
\hline $\mathbf{C}-\mathbf{C}(\mathbf{s p 3})$ & $12.7 \pm 0.3$ & $12.9 \pm 0.3$ & $10.9 \pm 0.3$ \\
\hline $\mathbf{C}-\mathbf{O H}$ & $7.6 \pm 0.3$ & $6.2 \pm 0.3$ & $11.9 \pm 0.3$ \\
\hline $\mathbf{C}-\mathbf{O}-\mathbf{C}$ & $40.3 \pm 0.8$ & $38,7 \pm 0.8$ & $27.4 \pm 0.6$ \\
\hline $\mathbf{C}=\mathbf{O}$ & $9.3 \pm 0.3$ & $10.1 \pm 0.3$ & $9.8 \pm 0.3$ \\
\hline $\mathbf{O}-\mathbf{C}=\mathbf{O}$ & $5.0 \pm 0.3$ & $4.4 \pm 0.3$ & $8.6 \pm 0.3$ \\
\hline
\end{tabular}



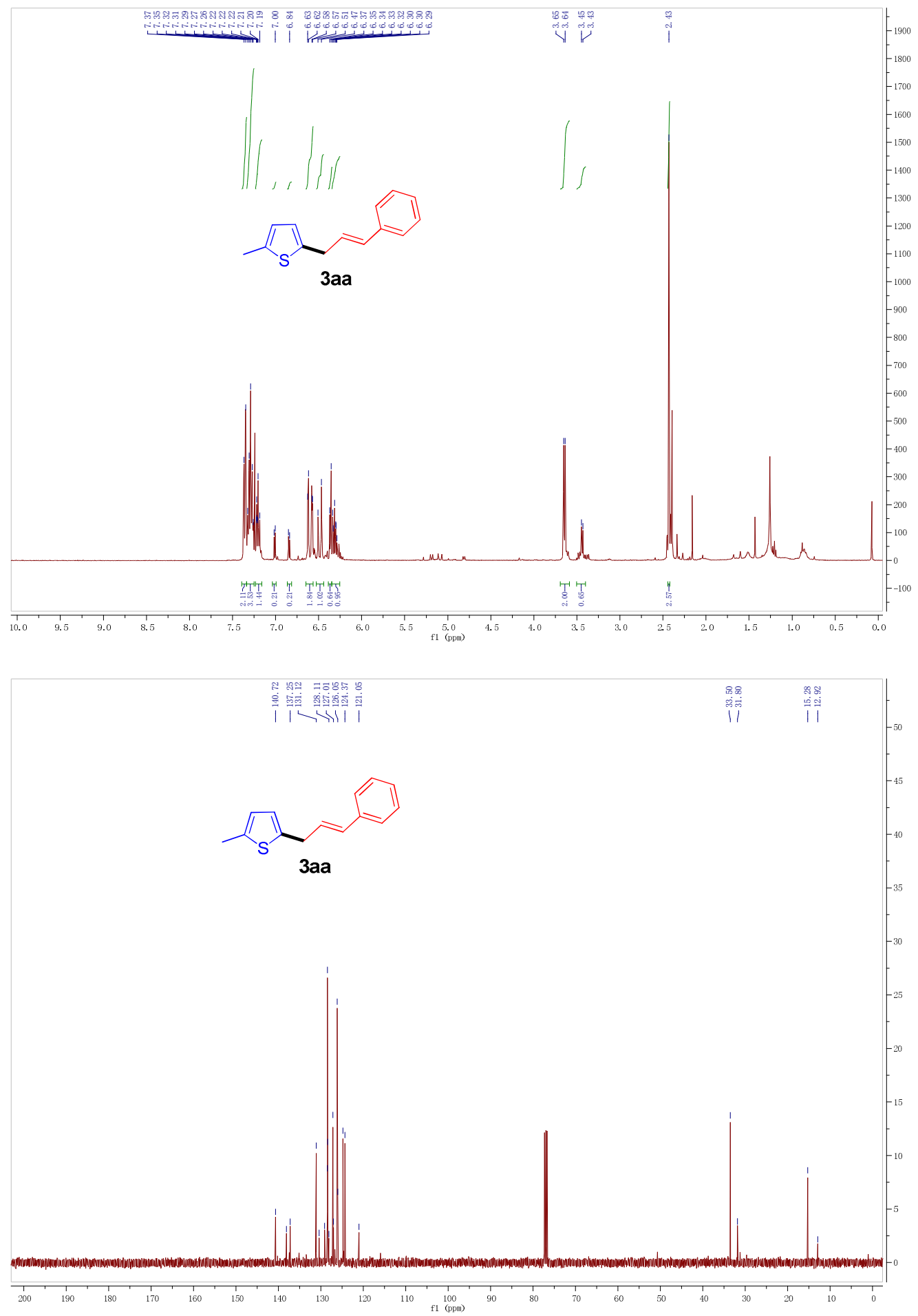

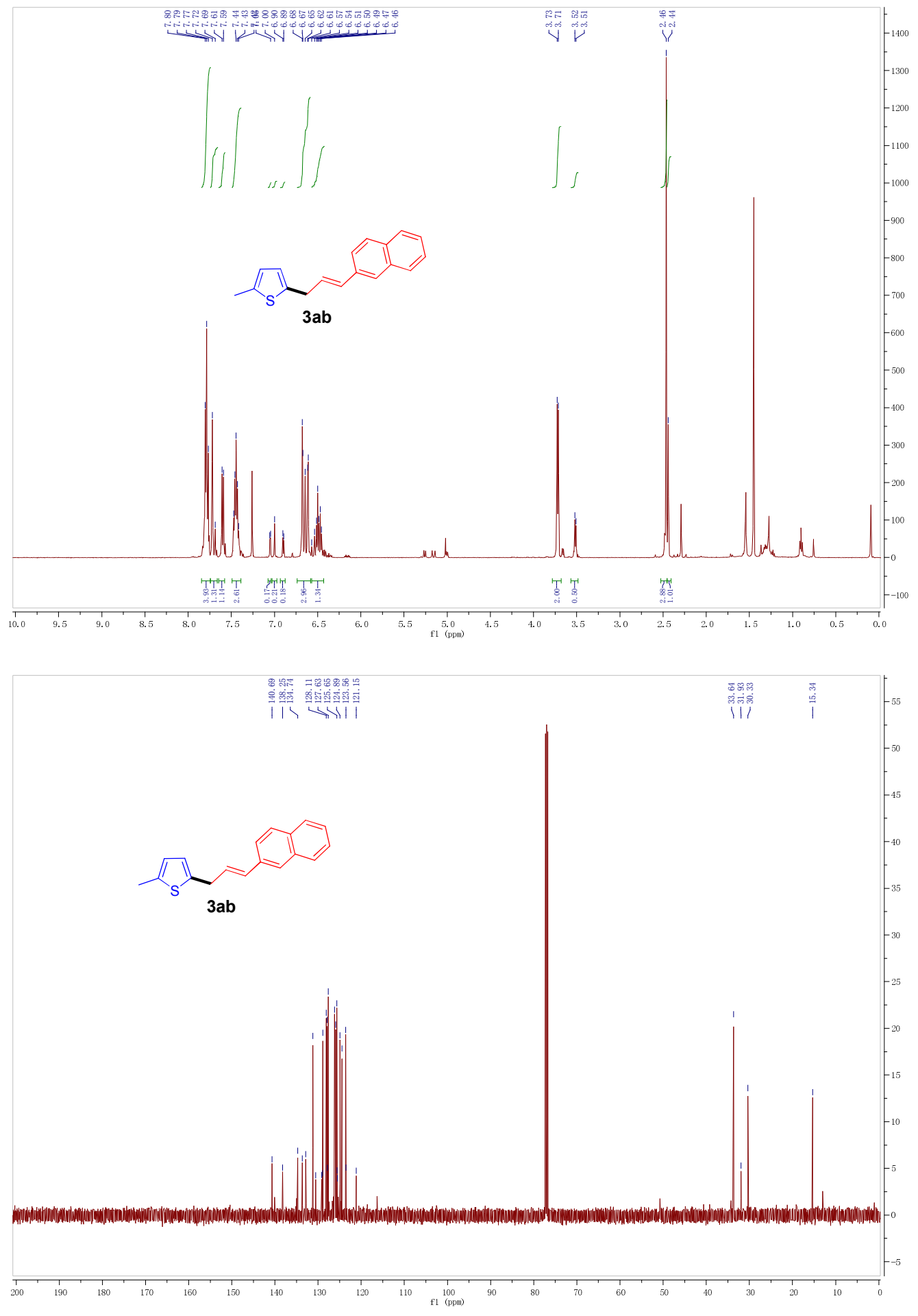


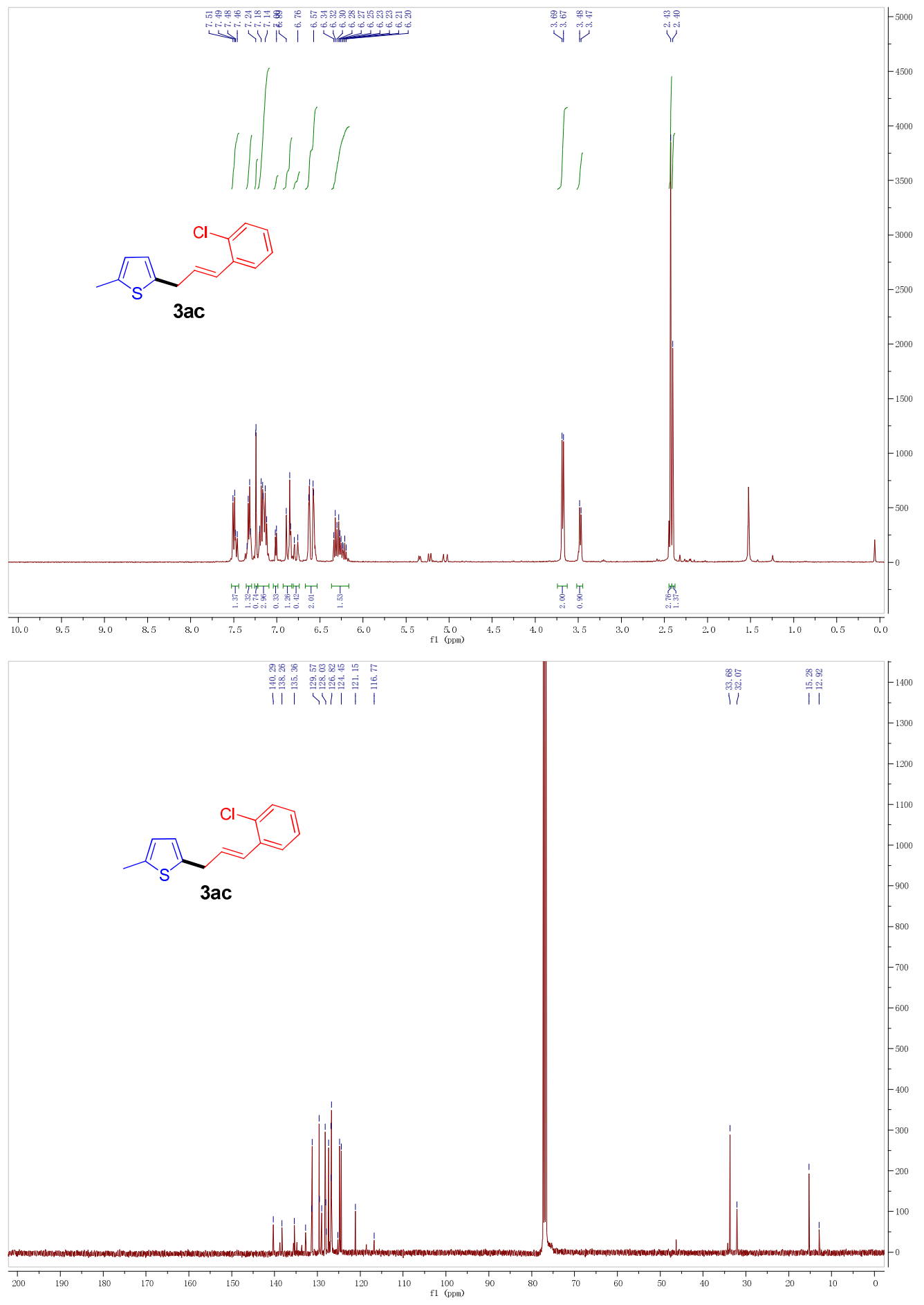




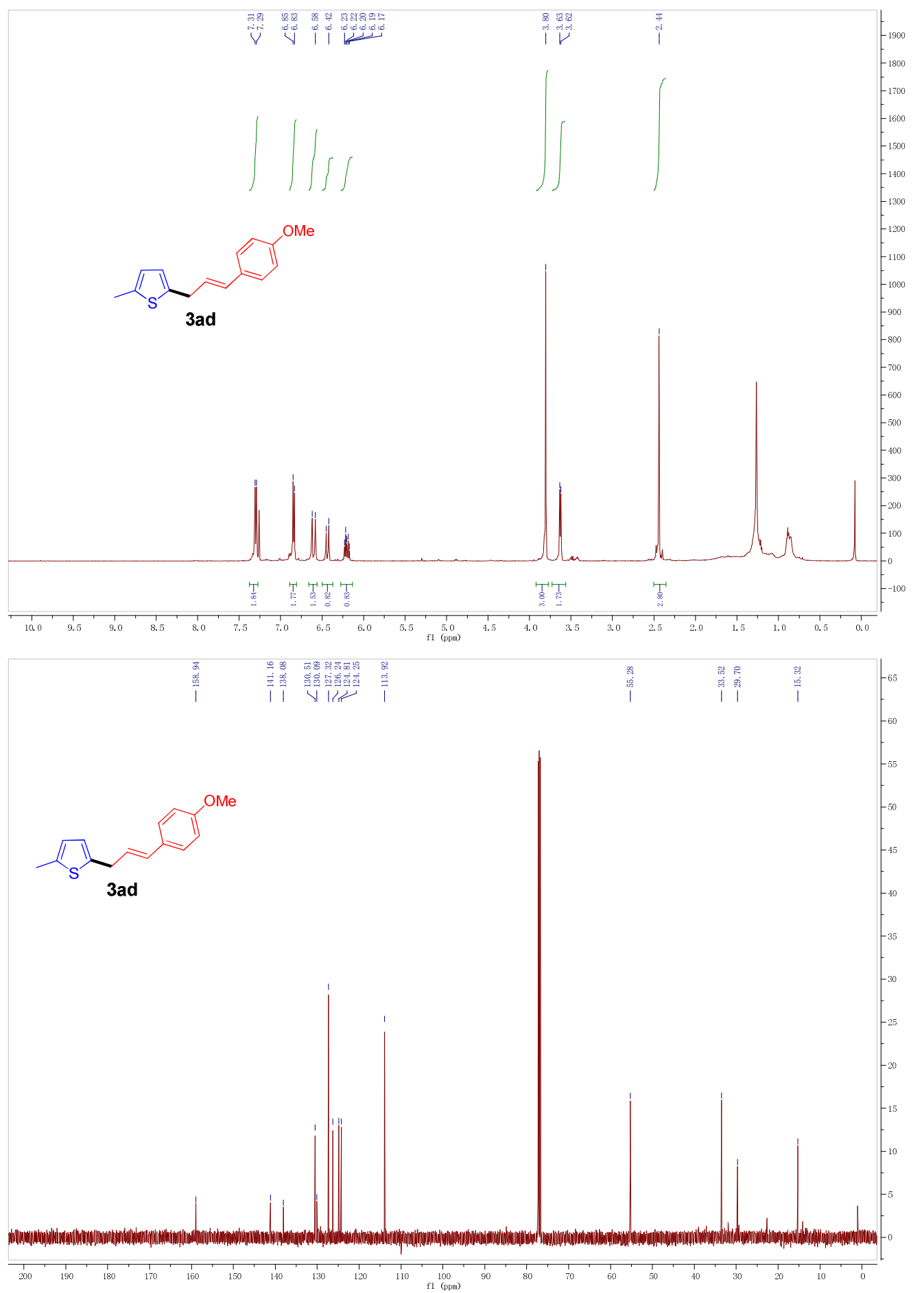



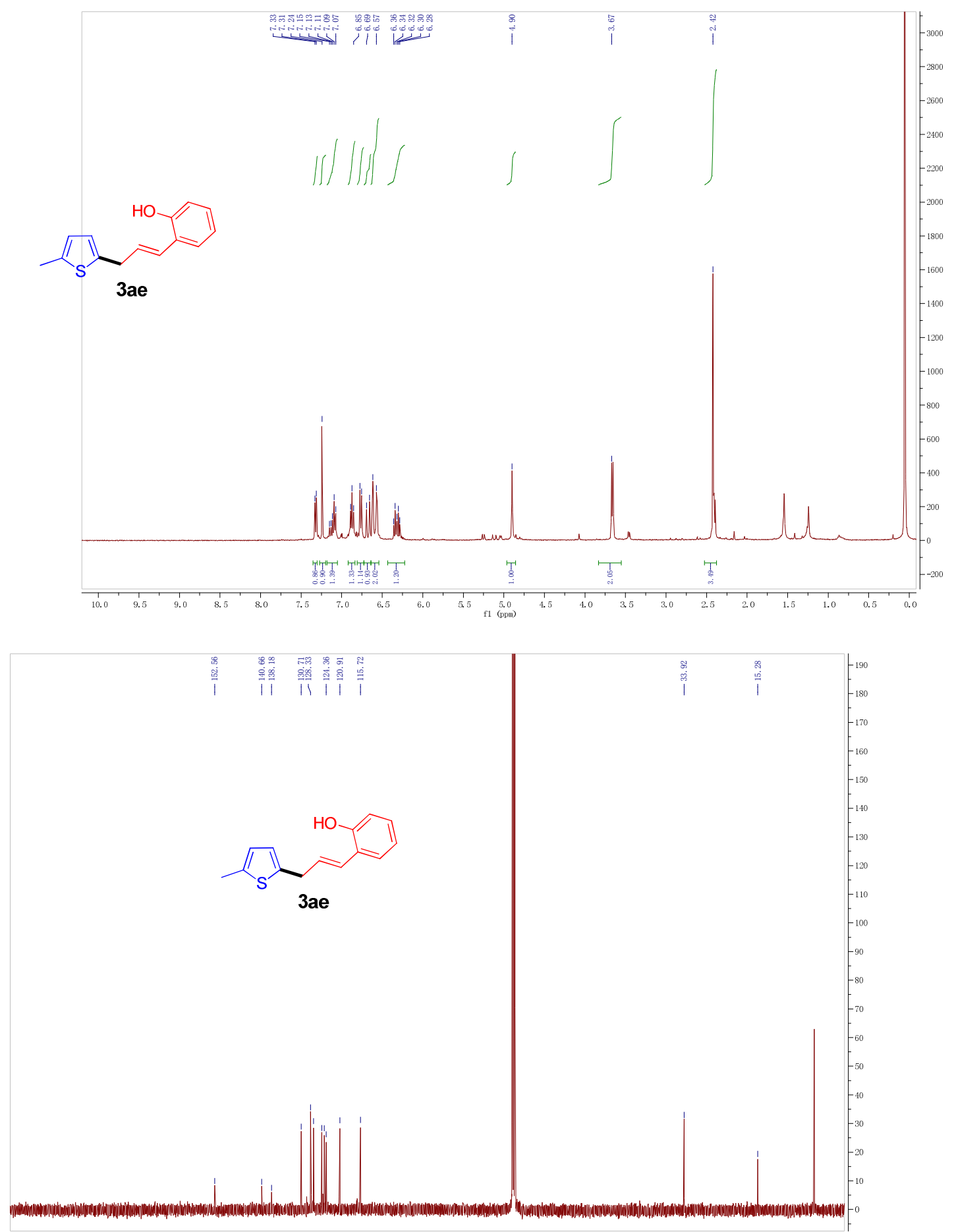

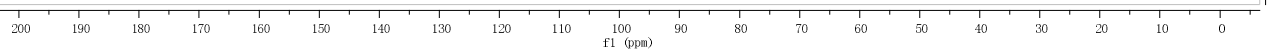




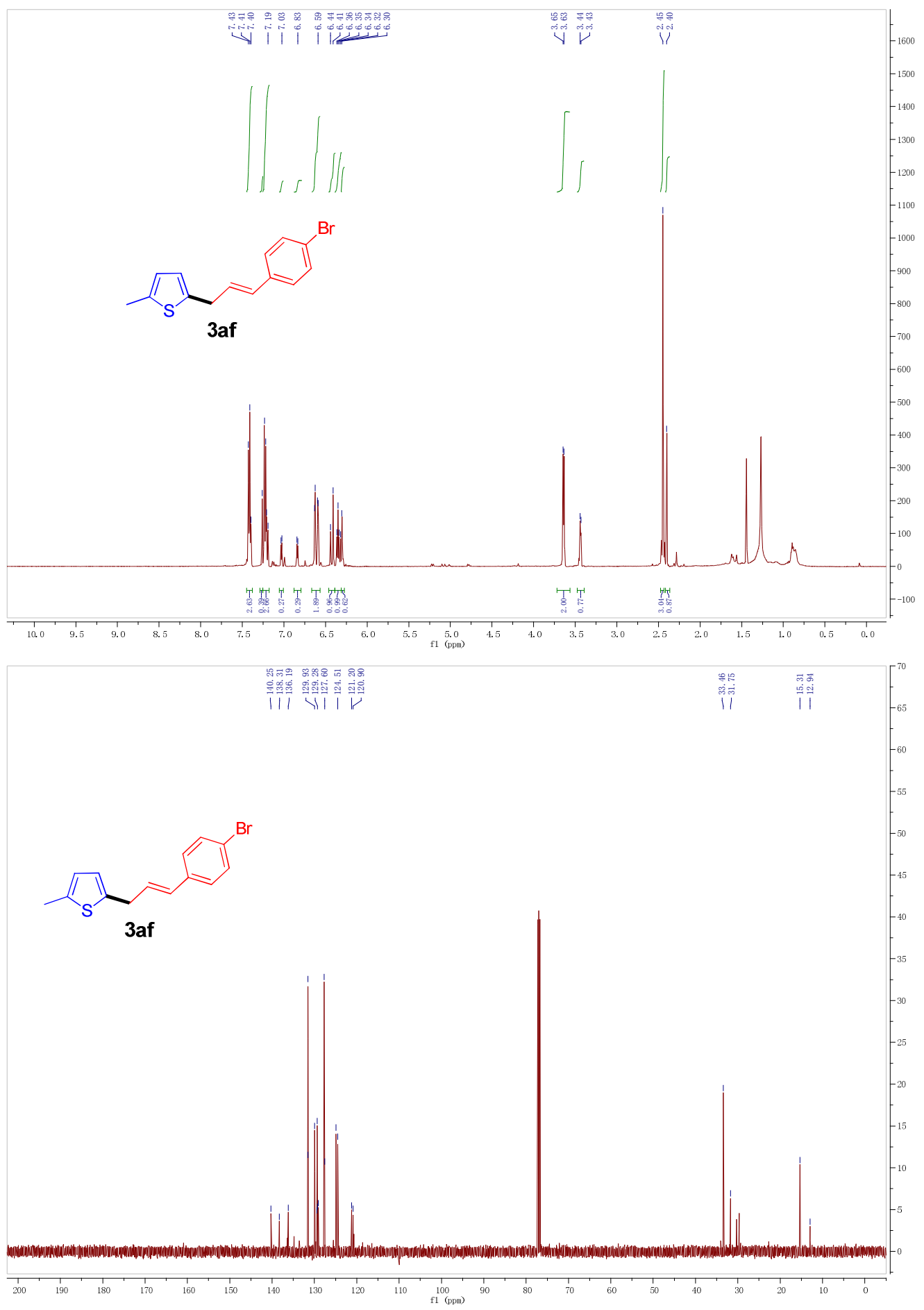



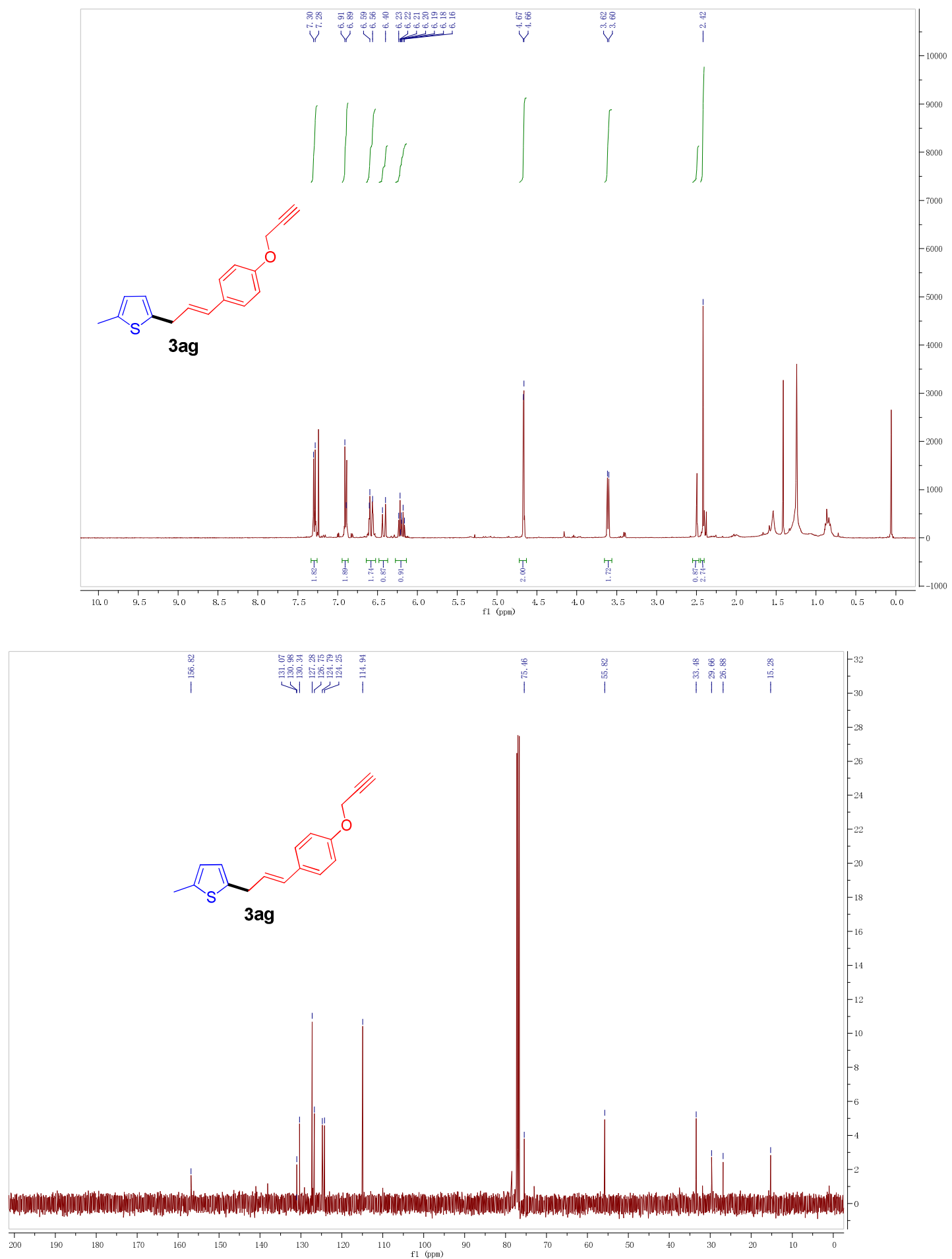

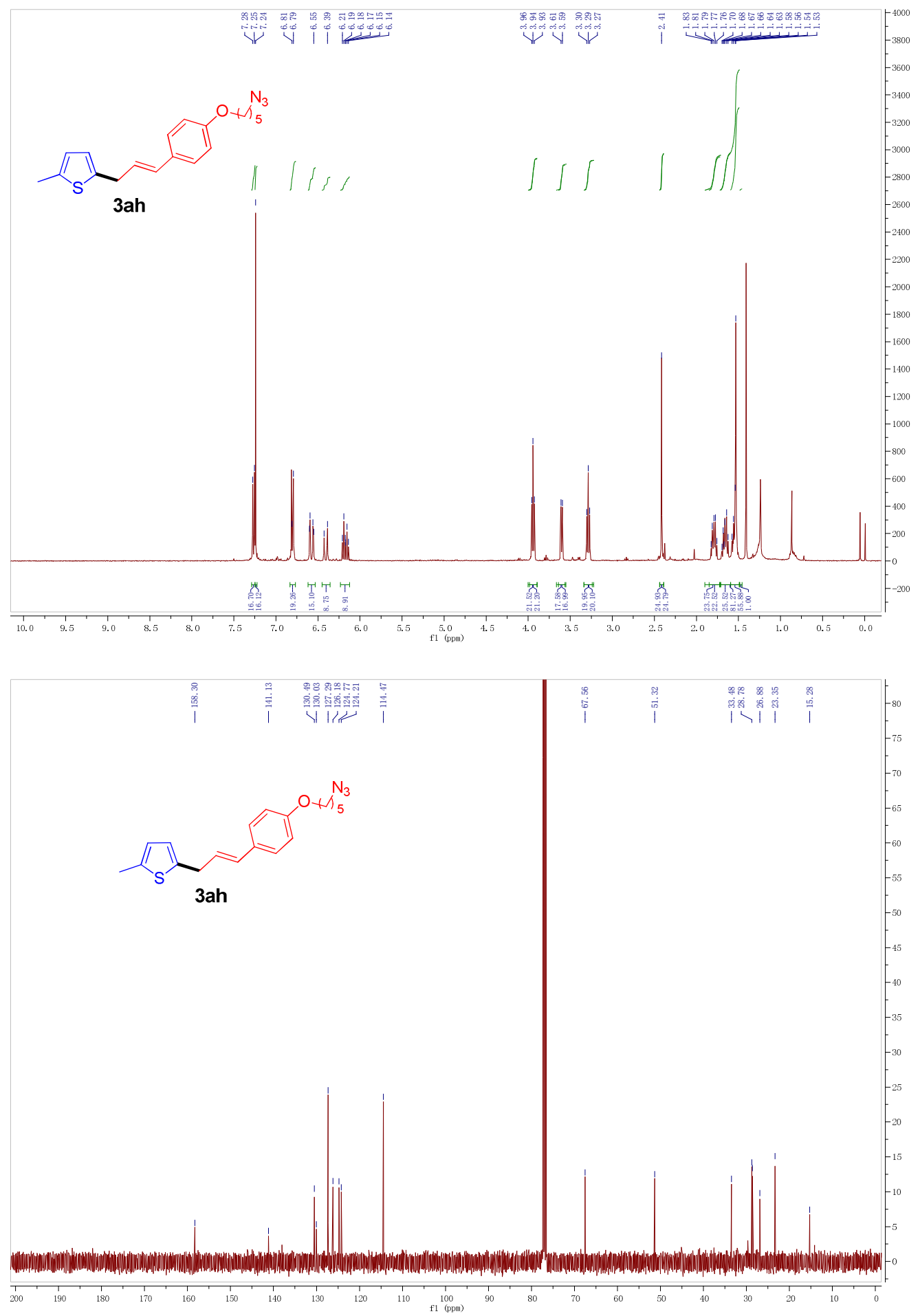

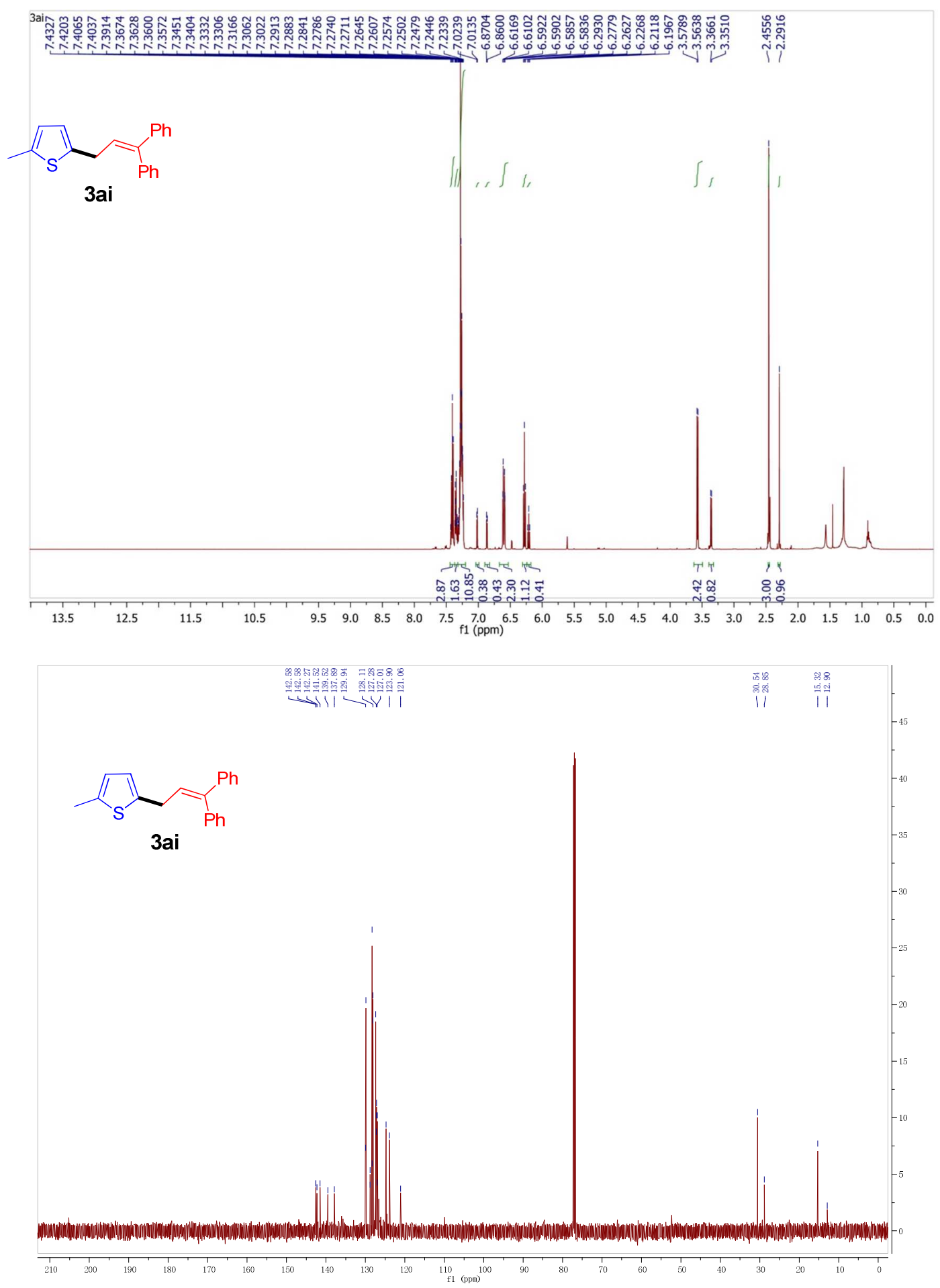


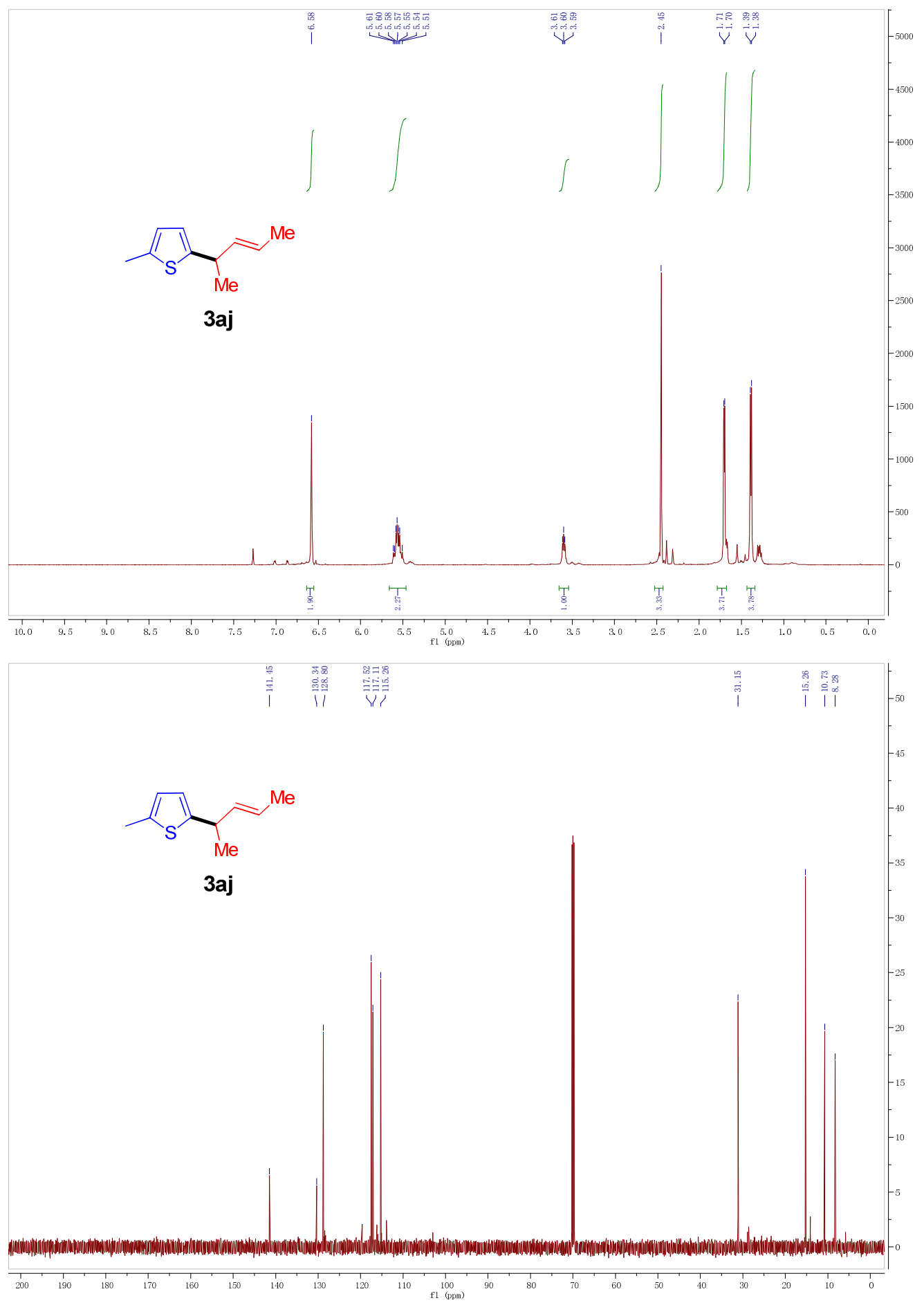




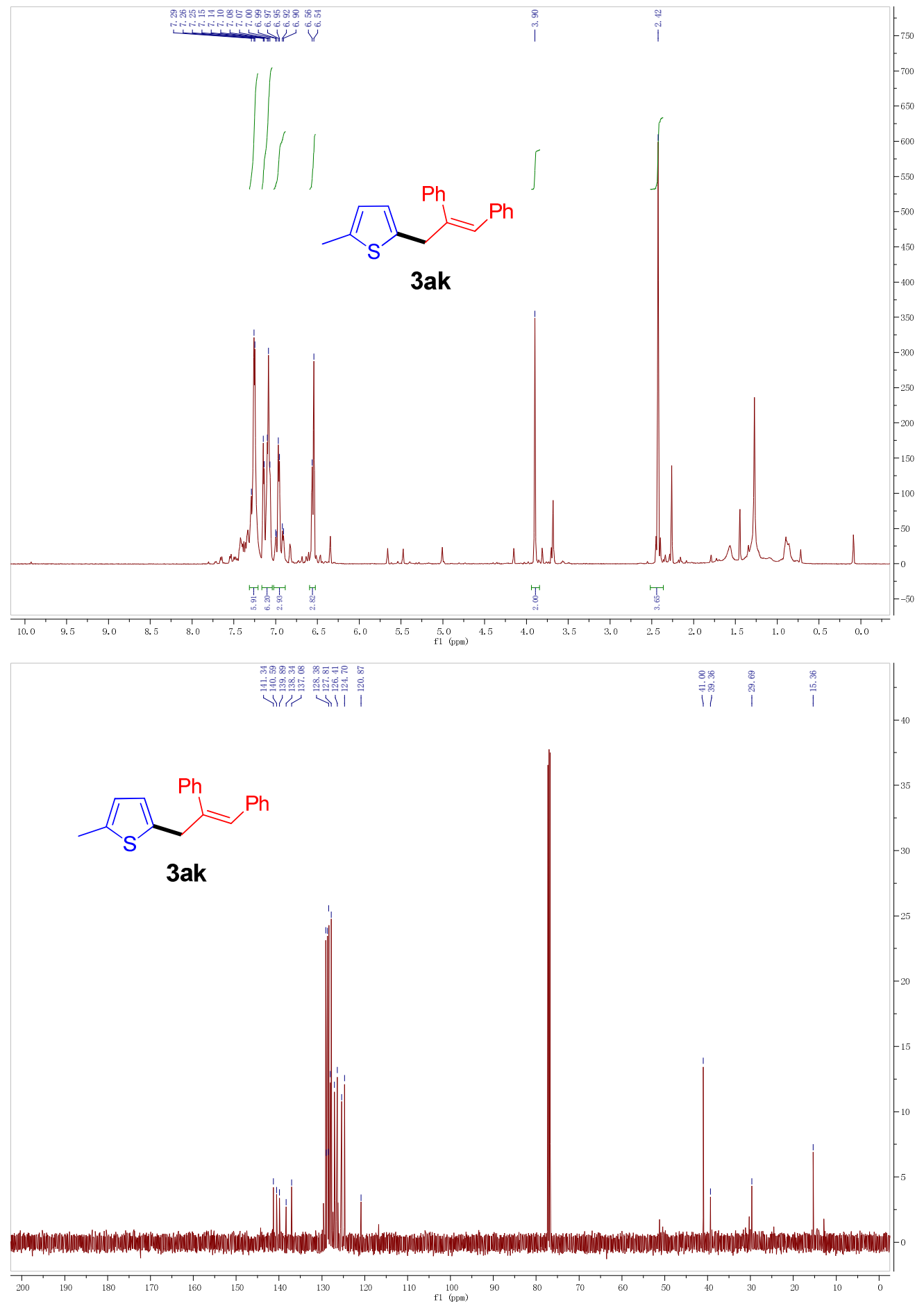




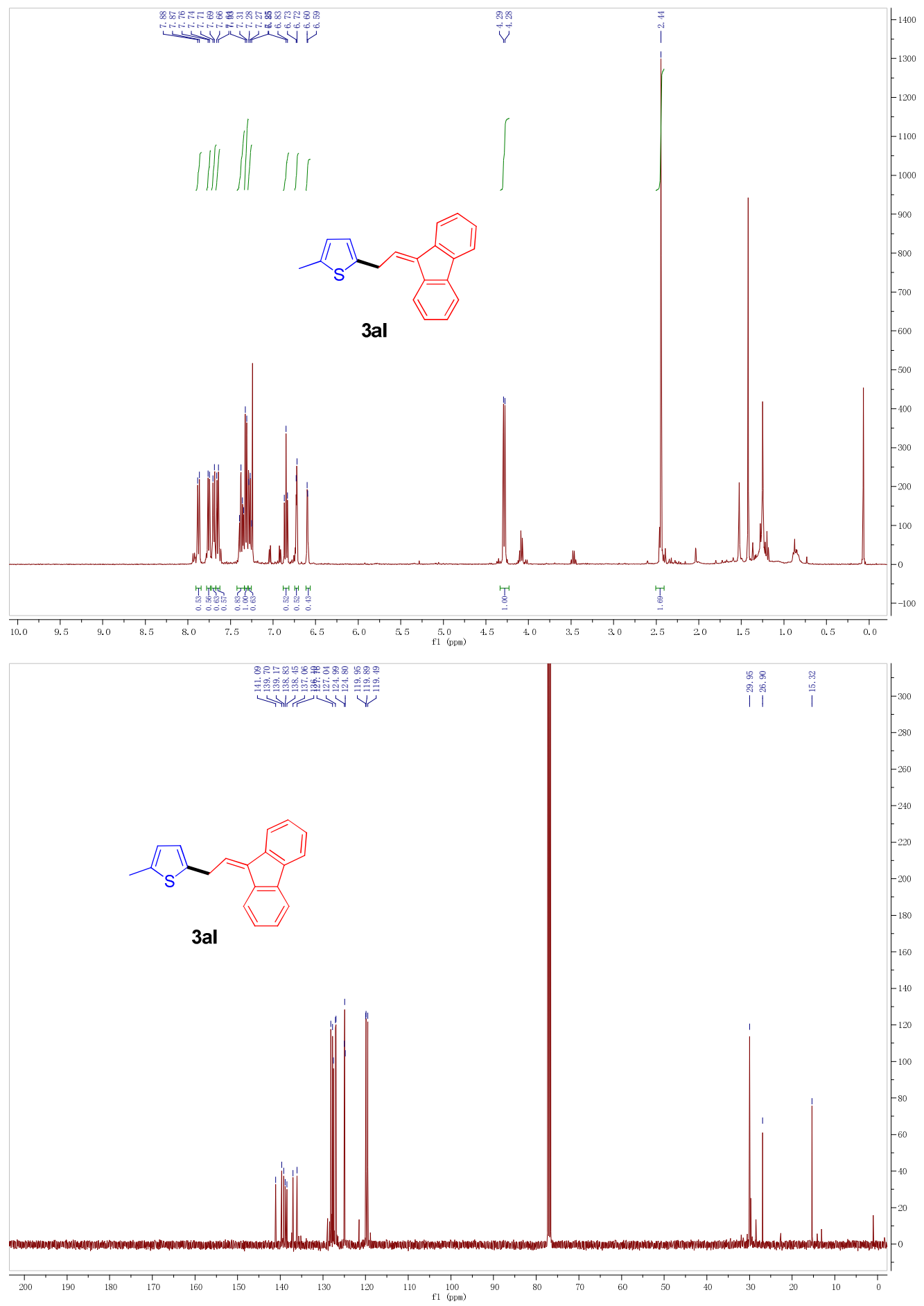




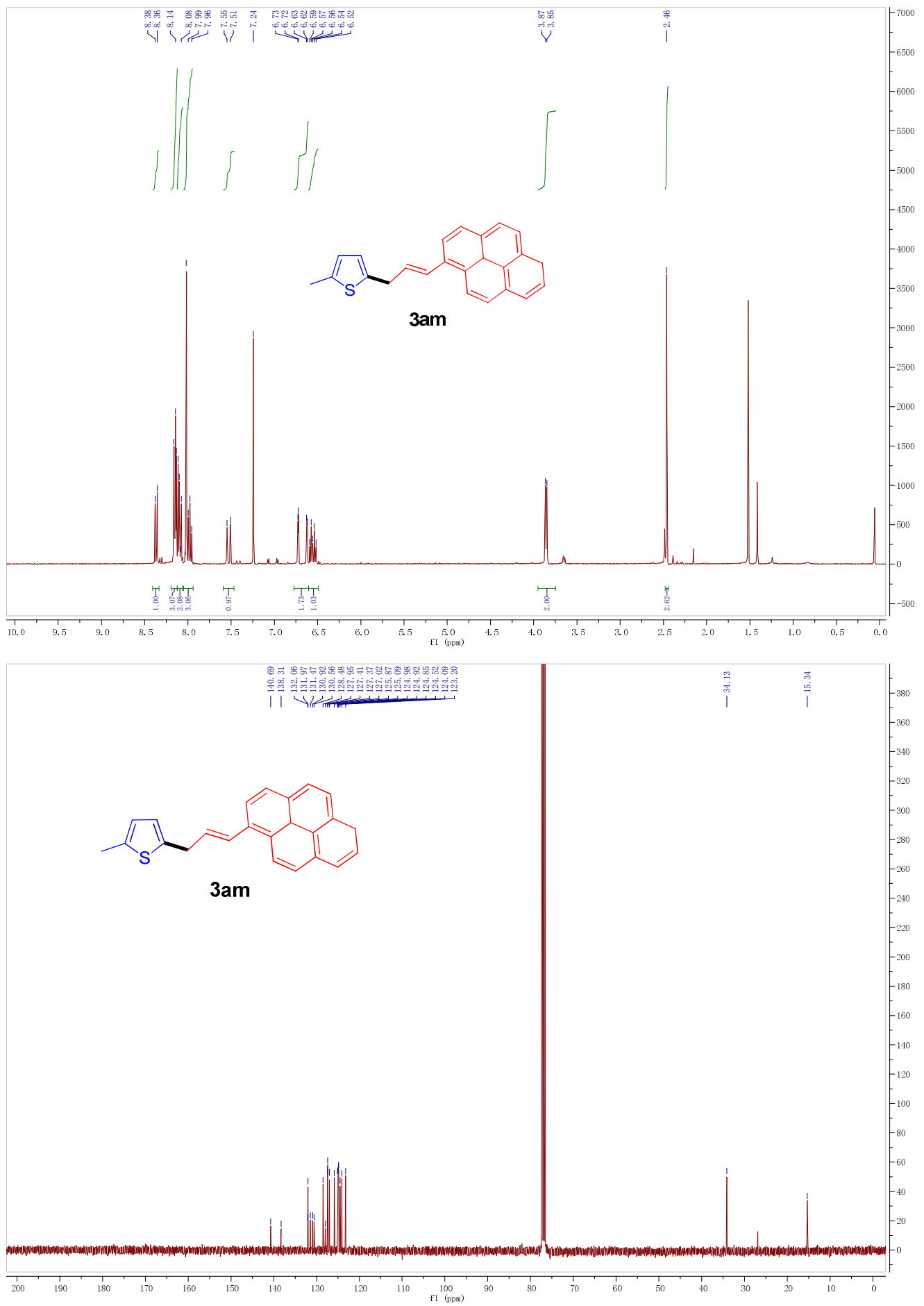



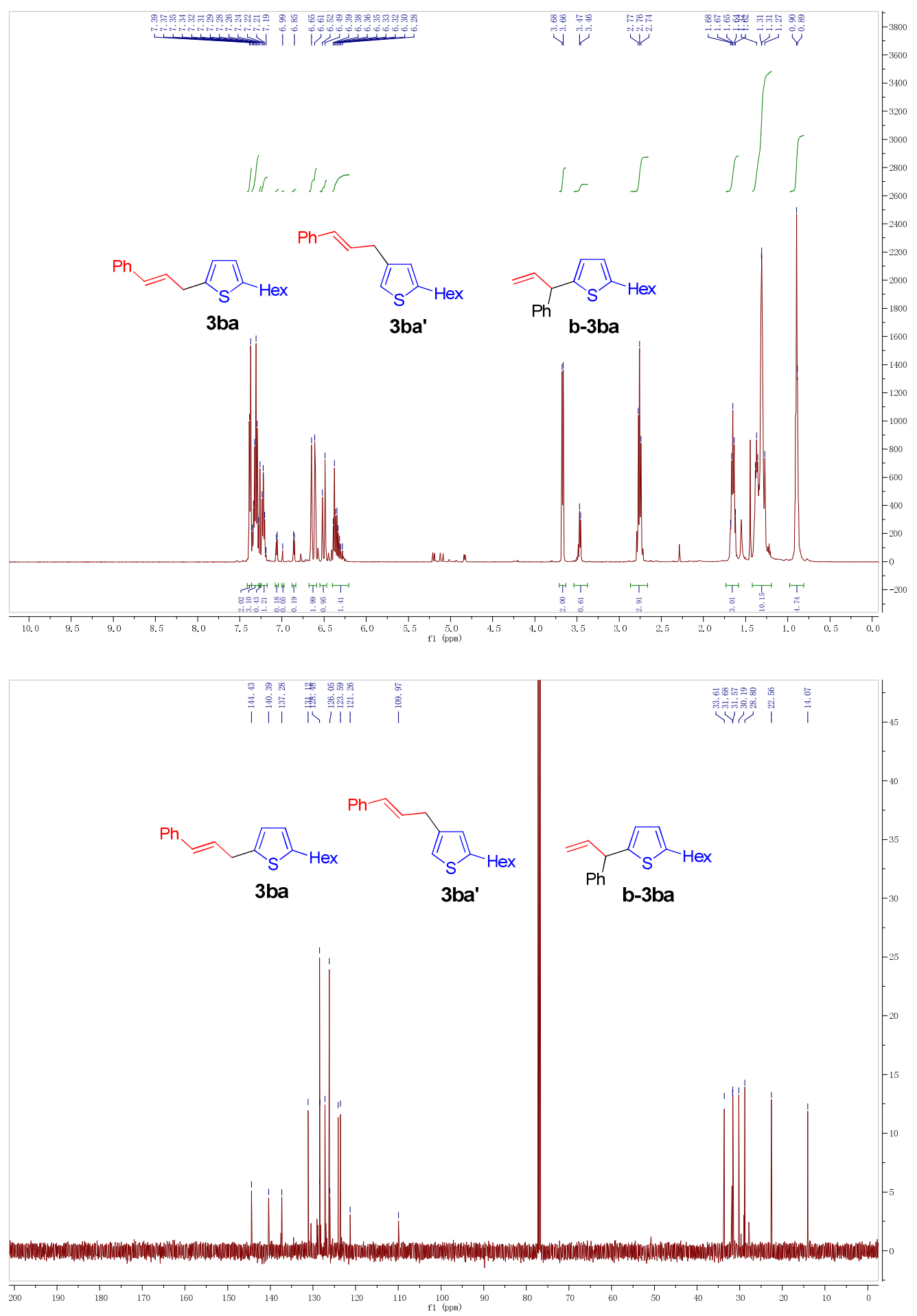

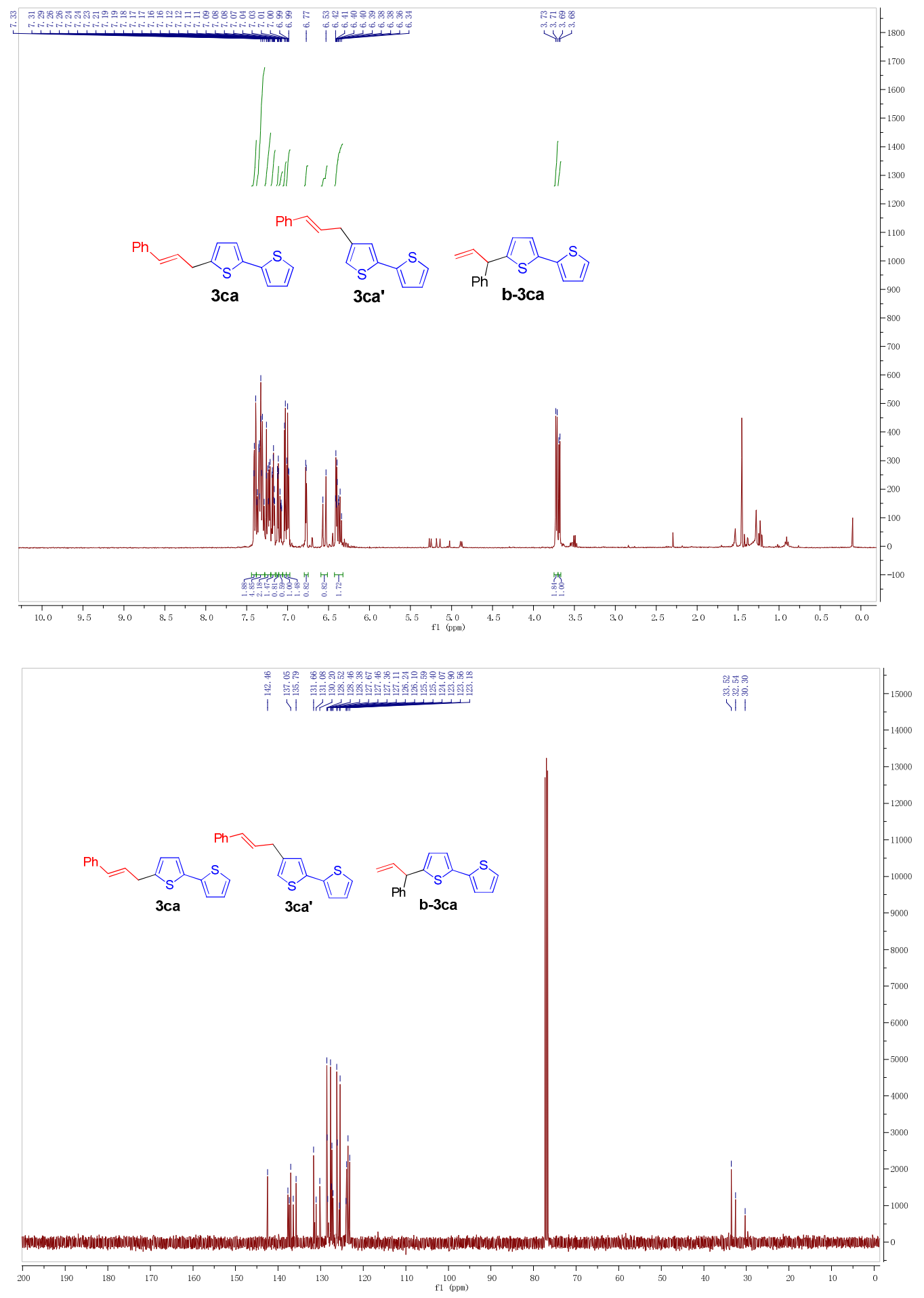


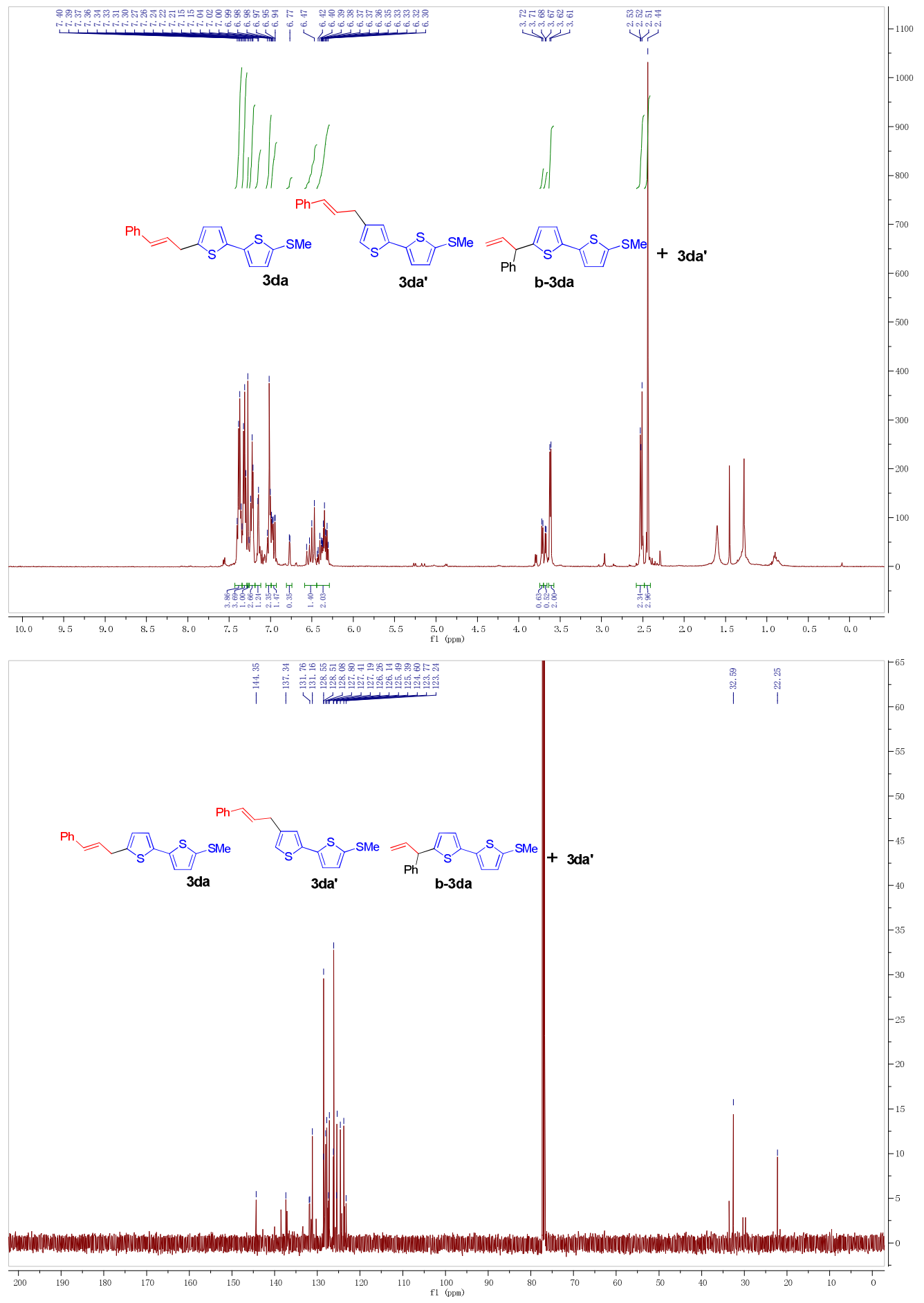




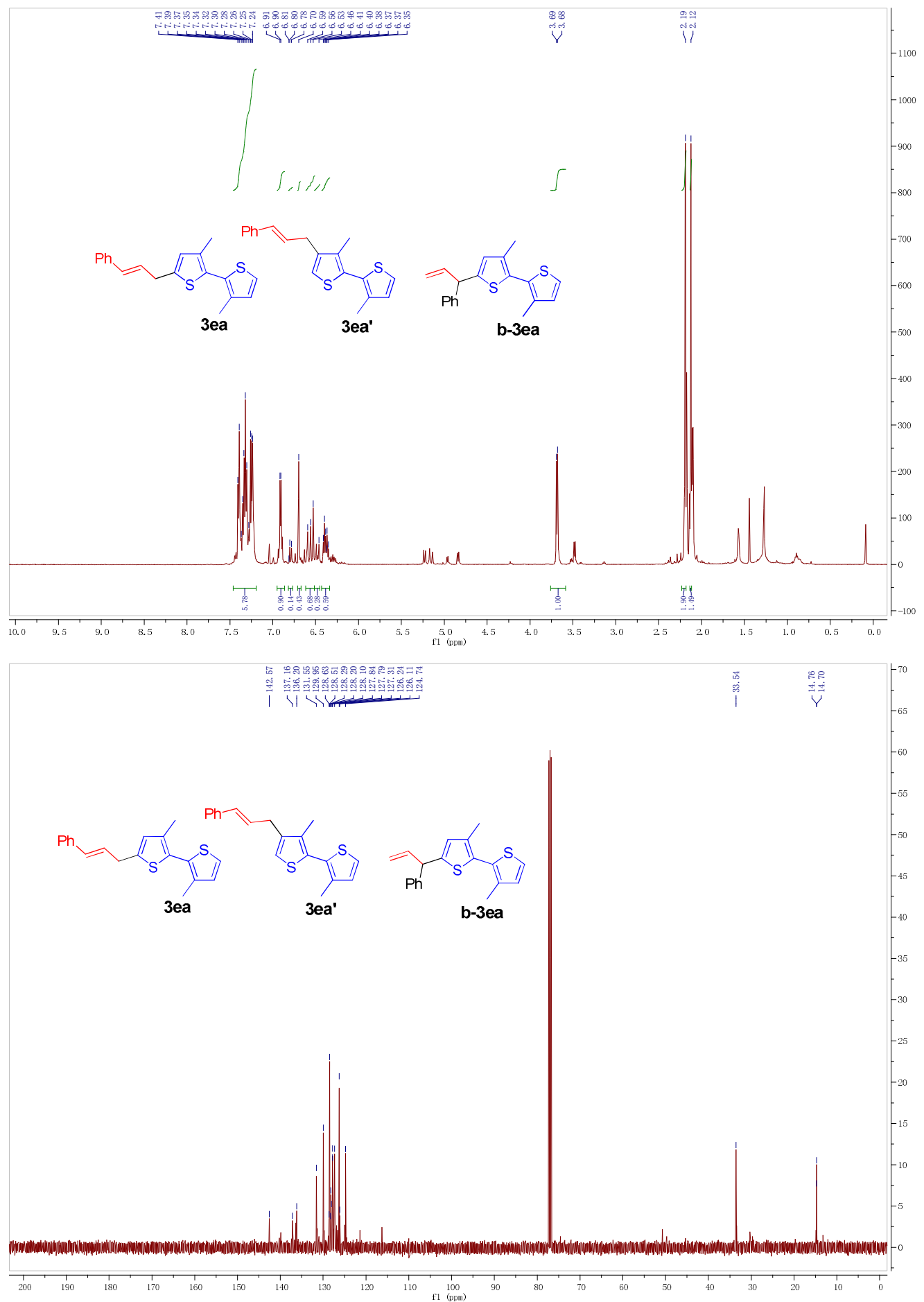




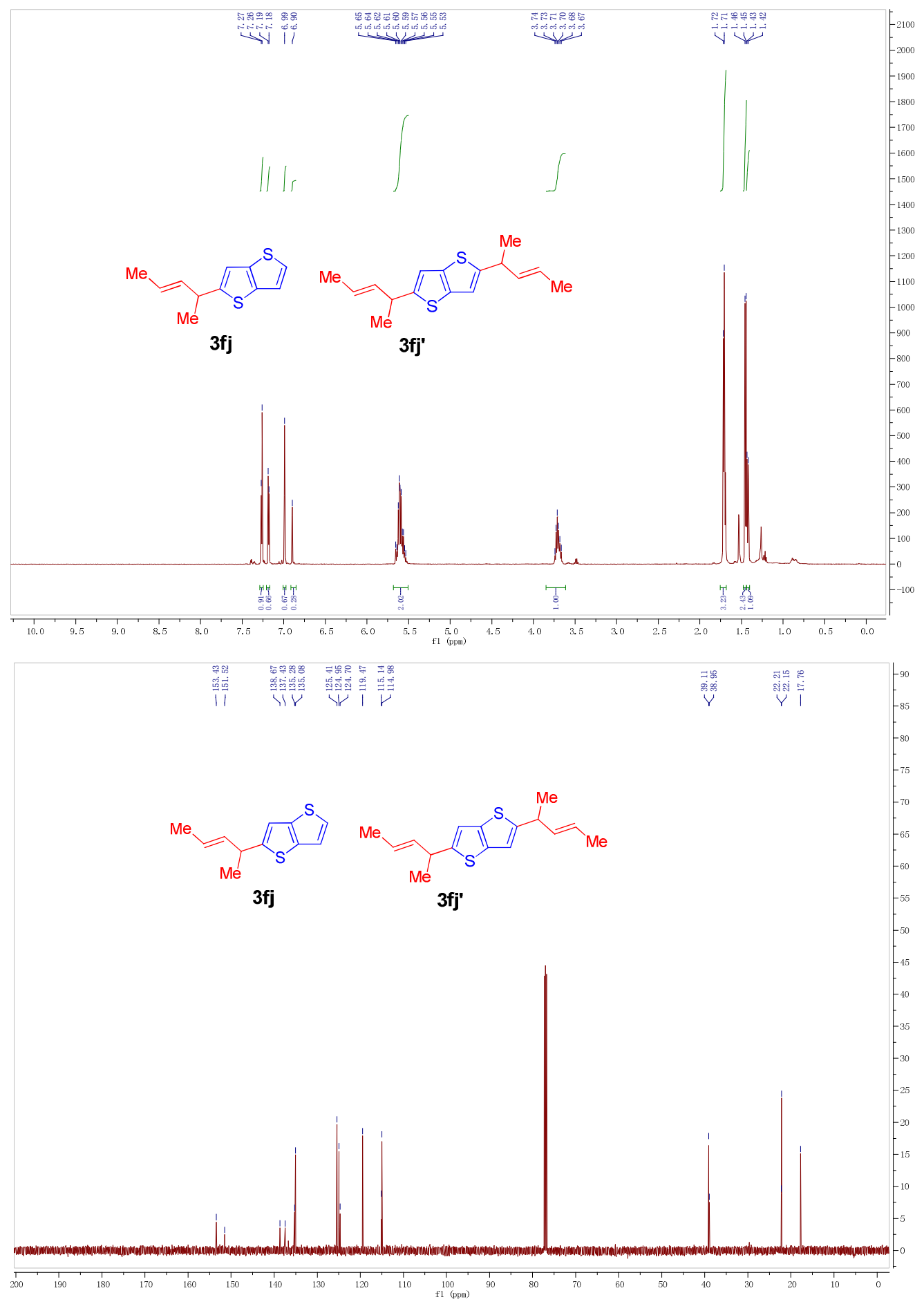




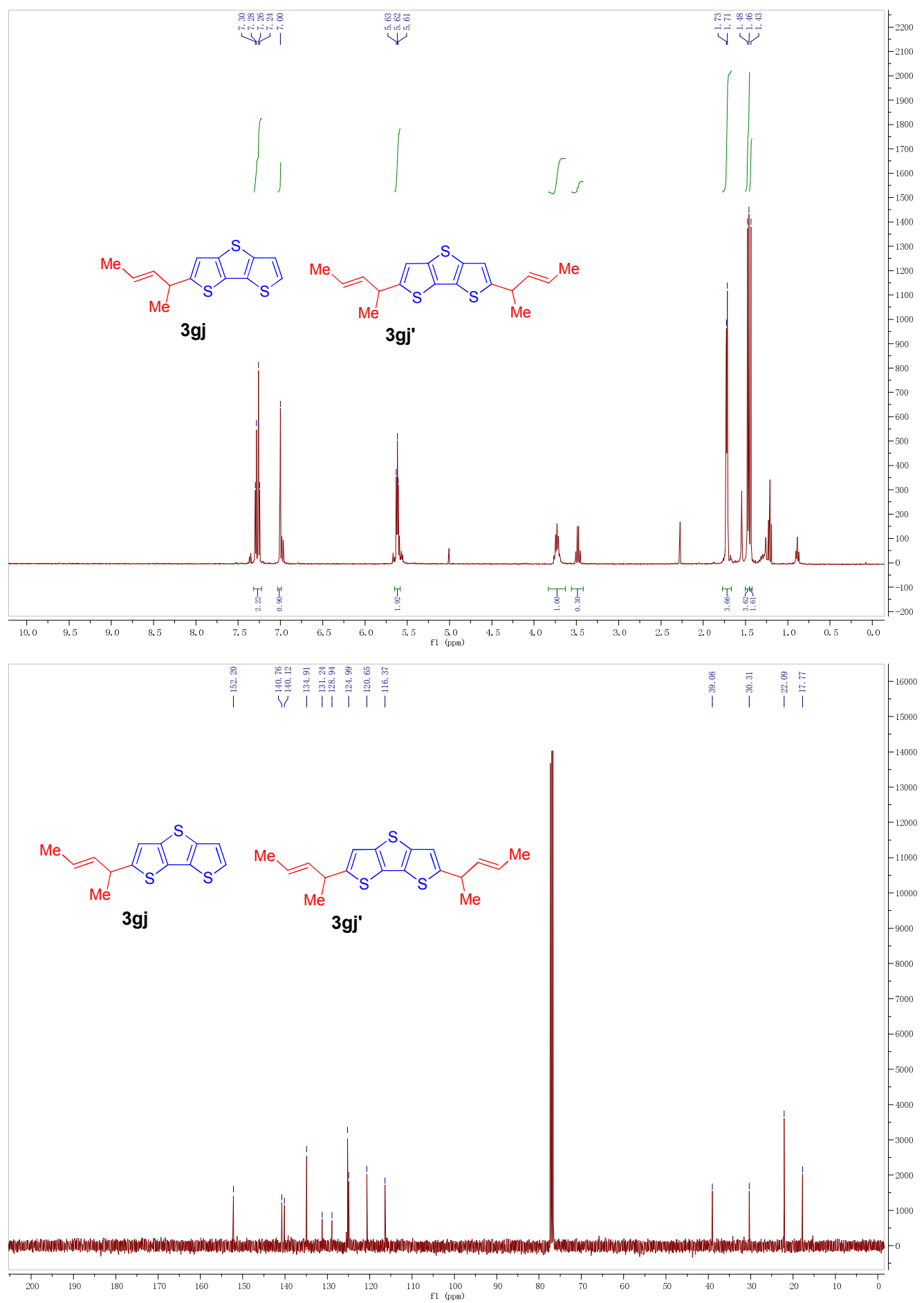




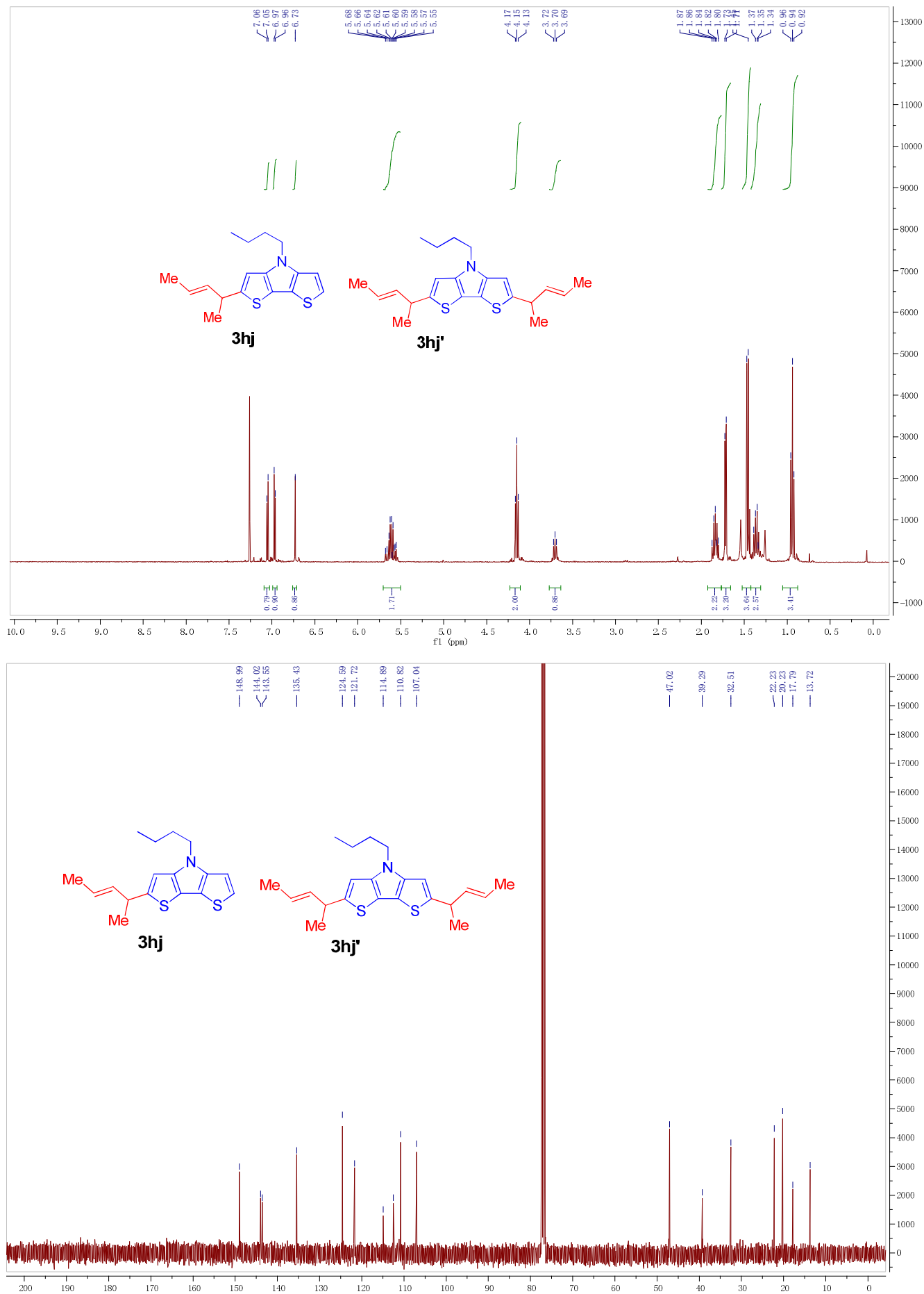




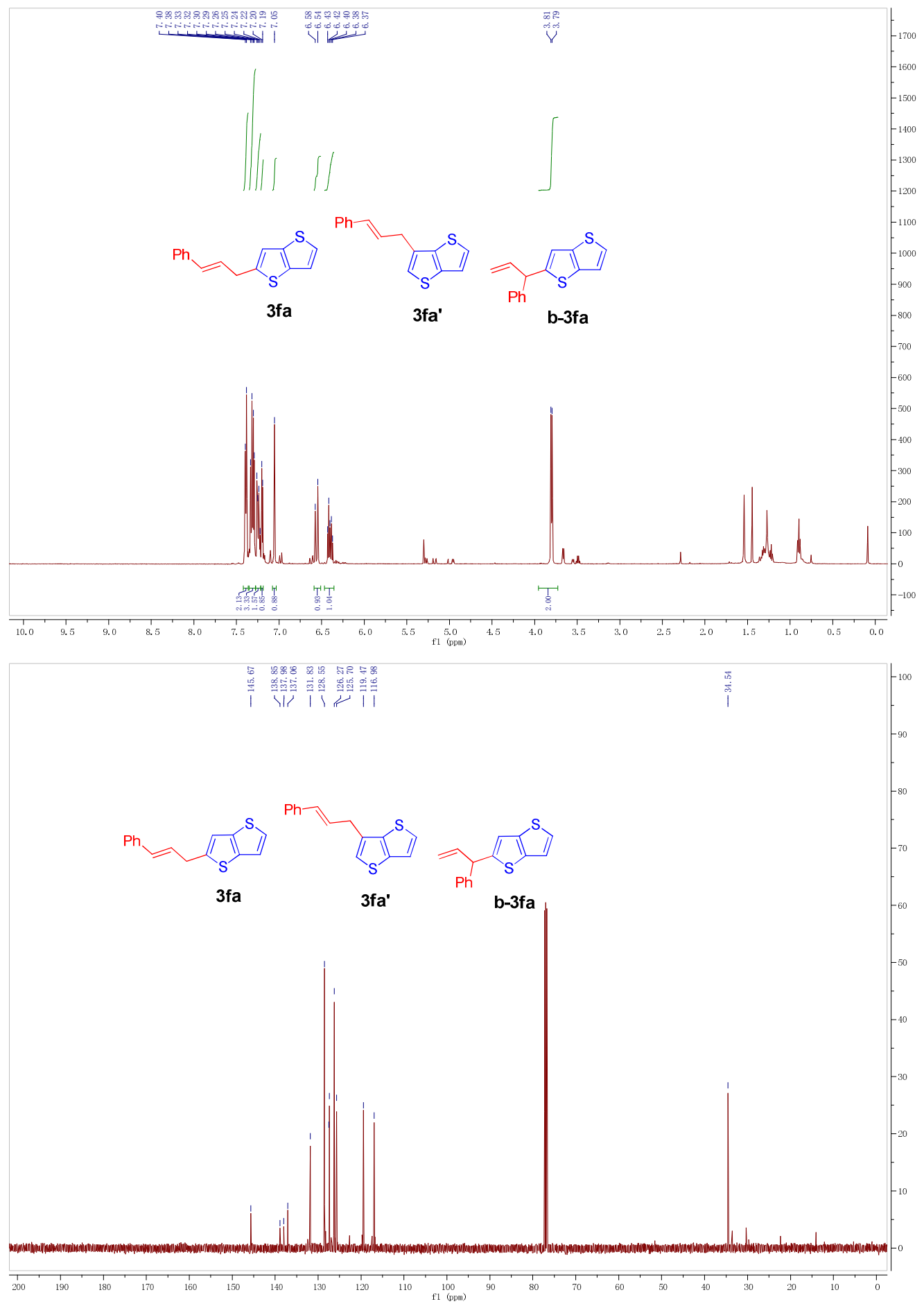



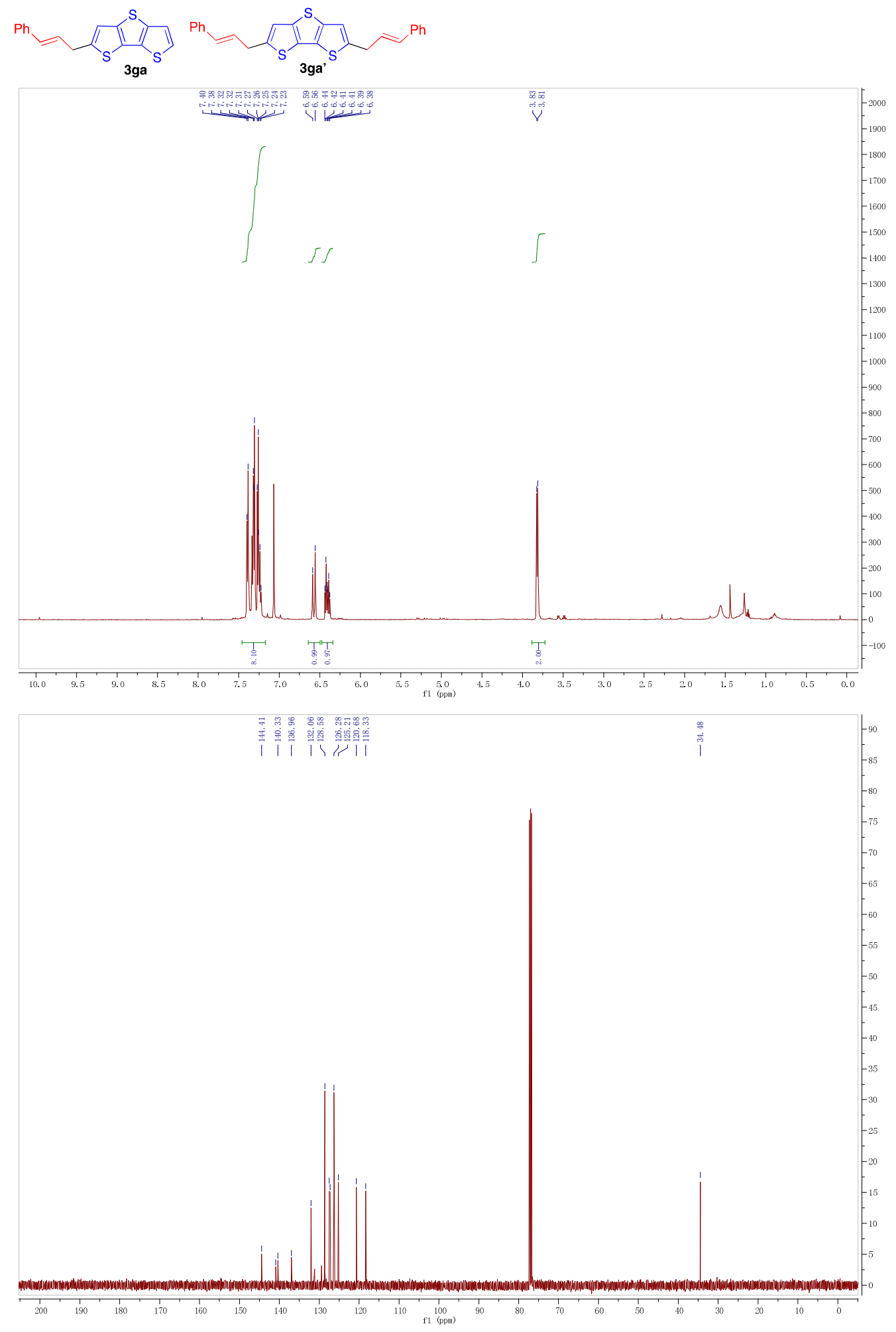


\section{Figure S2}

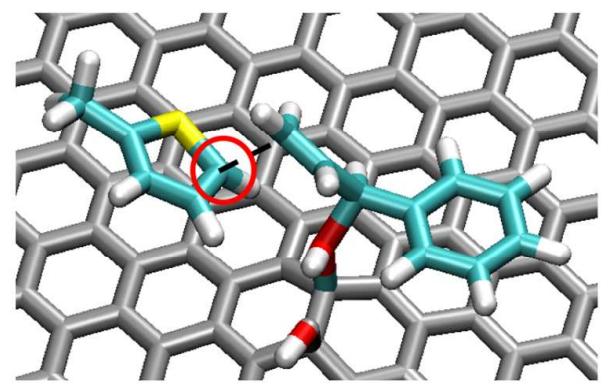

Attack $\alpha$
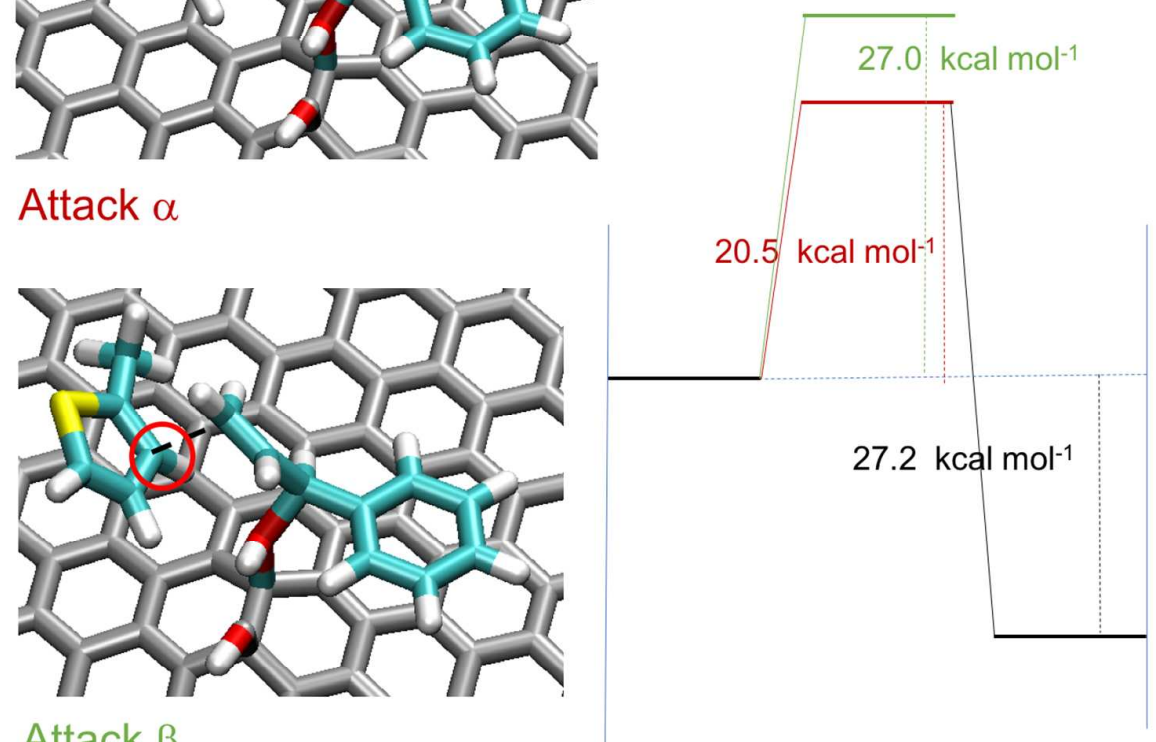

Attack $\beta$

Figure S2. TSs for the Friedel-Crafts-type allylic alkylation of the 2-methyl-thiophene in the $\alpha$ (red path) and $\beta$ (green path) position.

Table S2. Cartesian coordinates (Ångstroms) for the various computed critical points

\section{$\mathbf{R X 1 - 2 a}$}

$\begin{array}{rrrr}\mathrm{O} & 1.418715 & 0.698167 & 3.706447 \\ \mathrm{C} & 2.578360 & -0.050682 & 4.025263 \\ \mathrm{H} & 1.171454 & 0.477198 & 2.796047 \\ \mathrm{C} & 2.856992 & 0.173192 & 5.486147 \\ \mathrm{C} & 2.387158 & -1.534243 & 3.750831 \\ \mathrm{H} & 3.445104 & 0.305449 & 3.445867 \\ \mathrm{C} & 3.489615 & -2.347682 & 3.486773 \\ \mathrm{C} & 3.324032 & -3.710985 & 3.268406 \\ \mathrm{C} & 2.050401 & -4.275428 & 3.314281 \\ \mathrm{C} & 0.947577 & -3.469679 & 3.582213 \\ \mathrm{C} & 1.115073 & -2.104304 & 3.801752 \\ \mathrm{H} & 4.483712 & -1.906599 & 3.454005 \\ \mathrm{H} & 4.188162 & -4.334324 & 3.058634 \\ \mathrm{H} & 1.920085 & -5.339307 & 3.141376 \\ \mathrm{H} & -0.046871 & -3.904237 & 3.622632\end{array}$




$\begin{array}{rrrr}\mathrm{H} & 0.260275 & -1.468524 & 4.014289 \\ \mathrm{C} & 4.025871 & 0.585360 & 5.962307 \\ \mathrm{H} & 2.025791 & -0.059858 & 6.150328 \\ \mathrm{H} & 4.199551 & 0.703636 & 7.026895 \\ \mathrm{H} & 4.854943 & 0.821850 & 5.299507\end{array}$

\section{RX1-GO}

\begin{tabular}{|c|c|c|c|}
\hline C & 10.314505 & 9.421254 & -0.241179 \\
\hline $\mathrm{C}$ & 9.080800 & 10.059696 & -0.278548 \\
\hline $\mathrm{C}$ & 10.386992 & 8.030645 & -0.186472 \\
\hline $\mathrm{C}$ & 9.213802 & 7.241255 & -0.165262 \\
\hline C & 10.459792 & 5.021157 & -0.083343 \\
\hline $\mathrm{C}$ & 9.248609 & 5.743398 & -0.112653 \\
\hline $\mathrm{C}$ & 10.484335 & 3.683629 & -0.049397 \\
\hline $\mathrm{C}$ & 9.300676 & 2.921051 & -0.045311 \\
\hline $\mathrm{C}$ & 10.497663 & 0.670935 & -0.169399 \\
\hline $\mathrm{C}$ & 9.310675 & 1.415515 & -0.041498 \\
\hline $\mathrm{C}$ & 10.491962 & -0.666486 & -0.172396 \\
\hline $\mathrm{C}$ & 9.297997 & -1.408308 & -0.043799 \\
\hline $\mathrm{C}$ & 10.468343 & -3.667075 & -0.096701 \\
\hline $\mathrm{C}$ & 9.283909 & -2.907246 & -0.053110 \\
\hline $\mathrm{C}$ & 10.449965 & -5.001492 & -0.155767 \\
\hline $\mathrm{C}$ & 9.242356 & -5.732794 & -0.173897 \\
\hline C & 10.388434 & -7.944032 & -0.402604 \\
\hline $\mathrm{C}$ & 9.214121 & -7.157057 & -0.276375 \\
\hline $\mathrm{C}$ & 10.320700 & -9.332325 & -0.505278 \\
\hline $\mathrm{C}$ & 9.093066 & -9.980292 & -0.491349 \\
\hline $\mathrm{C}$ & 7.899426 & 9.309774 & -0.258180 \\
\hline $\mathrm{C}$ & 6.665222 & 9.966248 & -0.297309 \\
\hline $\mathrm{C}$ & 7.957006 & 7.892943 & -0.198136 \\
\hline $\mathrm{C}$ & 6.756824 & 7.144295 & -0.168061 \\
\hline $\mathrm{C}$ & 8.028265 & 5.026950 & -0.083610 \\
\hline $\mathrm{C}$ & 6.792637 & 5.727411 & -0.101902 \\
\hline $\mathrm{C}$ & 8.064789 & 3.604894 & -0.036604 \\
\hline $\mathrm{C}$ & 6.807748 & 2.828052 & 0.041570 \\
\hline C & 8.088183 & 0.713361 & 0.054534 \\
\hline C & 6.820266 & 1.474428 & 0.108817 \\
\hline $\mathrm{C}$ & 8.073303 & -0.711165 & 0.059565 \\
\hline $\mathrm{C}$ & 6.854224 & -1.433884 & 0.146191 \\
\hline $\mathrm{C}$ & 8.045568 & -3.585879 & -0.012062 \\
\hline $\mathrm{C}$ & 6.837541 & -2.856934 & 0.099760 \\
\hline $\mathrm{C}$ & 8.023106 & -5.006655 & -0.078255 \\
\hline $\mathrm{C}$ & 6.788636 & -5.692025 & -0.052709 \\
\hline $\mathrm{C}$ & 7.962420 & -7.828915 & -0.265897 \\
\hline $\mathrm{C}$ & 6.758541 & -7.099063 & -0.149827 \\
\hline $\mathrm{C}$ & 7.911436 & -9.242682 & -0.374881 \\
\hline C & 6.680925 & -9.906966 & -0.372702 \\
\hline C & 5.471846 & 9.240460 & -0.272626 \\
\hline C & 4.243312 & 9.920348 & -0.316168 \\
\hline $\mathrm{C}$ & 5.513421 & 7.829282 & -0.203121 \\
\hline C & 4.236576 & 7.070918 & -0.162278 \\
\hline $\mathrm{C}$ & 5.508584 & 4.972059 & -0.043776 \\
\hline $\mathrm{C}$ & 4.274075 & 5.664166 & -0.071842 \\
\hline C & 5.523032 & 3.563851 & 0.049554 \\
\hline $\mathrm{C}$ & 4.320300 & 2.846956 & 0.153561 \\
\hline $\mathrm{C}$ & 5.552262 & 0.723504 & 0.228857 \\
\hline $\mathrm{C}$ & 4.339496 & 1.444700 & 0.260123 \\
\hline $\mathrm{C}$ & 5.566834 & -0.694022 & 0.268176 \\
\hline C & 4.371398 & -1.401704 & 0.383358 \\
\hline C & 5.607121 & -3.562887 & 0.162011 \\
\hline $\mathrm{C}$ & 4.326941 & -2.823787 & 0.324900 \\
\hline C & 5.580656 & -4.970949 & 0.072010 \\
\hline C & 4.344202 & -5.659568 & 0.099244 \\
\hline $\mathrm{C}$ & 5.519358 & -7.782288 & -0.141967 \\
\hline $\mathrm{C}$ & 4.311038 & -7.062036 & -0.021394 \\
\hline $\mathrm{C}$ & 5.487637 & -9.189221 & -0.260867 \\
\hline $\mathrm{C}$ & 4.262462 & -9.865241 & -0.275650 \\
\hline $\mathrm{C}$ & 3.030432 & 9.223301 & -0.287572 \\
\hline $\mathrm{C}$ & 1.804608 & 9.911077 & -0.332219 \\
\hline C & 3.049108 & 7.738146 & -0.201595 \\
\hline
\end{tabular}




\begin{tabular}{|c|c|c|c|}
\hline C & 1.824464 & 7.040279 & -0.154951 \\
\hline C & 3.065545 & 4.945416 & -0.002471 \\
\hline C & 1.831408 & 5.639863 & -0.050759 \\
\hline C & 3.098113 & 3.548499 & 0.118085 \\
\hline C & 1.826940 & 2.814144 & 0.240848 \\
\hline C & 3.079899 & 0.696474 & 0.452767 \\
\hline C & 1.838640 & 1.439981 & 0.459149 \\
\hline C & 3.105611 & -0.660610 & 0.546417 \\
\hline $\mathrm{C}$ & 1.900524 & -1.390224 & 0.692077 \\
\hline $\mathrm{C}$ & 3.138429 & -3.499322 & 0.377848 \\
\hline C & 1.872573 & -2.753320 & 0.566255 \\
\hline $\mathrm{C}$ & 3.070630 & -4.901205 & 0.247236 \\
\hline $\mathrm{C}$ & 1.872409 & -5.567737 & 0.232943 \\
\hline $\mathrm{C}$ & 3.076400 & -7.751546 & -0.036330 \\
\hline $\mathrm{C}$ & 1.858463 & -7.045576 & 0.069658 \\
\hline $\mathrm{C}$ & 3.059649 & -9.158170 & -0.173738 \\
\hline $\mathrm{C}$ & 1.840414 & -9.844406 & -0.218628 \\
\hline $\mathrm{C}$ & 0.585167 & 9.217246 & -0.292580 \\
\hline C & -0.641840 & 9.899822 & -0.330619 \\
\hline C & 0.593755 & 7.736173 & -0.198636 \\
\hline $\mathrm{C}$ & -0.629017 & 7.029707 & -0.144621 \\
\hline C & 0.601504 & 4.950473 & 0.000896 \\
\hline $\mathrm{C}$ & -0.620733 & 5.629910 & -0.034181 \\
\hline $\mathrm{C}$ & 0.617983 & 3.493467 & 0.173584 \\
\hline $\mathrm{C}$ & -0.580462 & 2.806430 & 0.284703 \\
\hline $\mathrm{C}$ & 0.636183 & 0.763050 & 0.612879 \\
\hline $\mathrm{C}$ & -0.574694 & 1.433046 & 0.509130 \\
\hline $\mathrm{C}$ & 0.644942 & -0.660938 & 1.063934 \\
\hline C & -0.624427 & -1.396874 & 0.730642 \\
\hline C & 0.636440 & -3.424371 & 0.542575 \\
\hline C & -0.601810 & -2.758948 & 0.601253 \\
\hline $\mathrm{C}$ & 0.637307 & -4.881739 & 0.338663 \\
\hline C & -0.609115 & -5.569316 & 0.250426 \\
\hline $\mathrm{C}$ & 0.629972 & -7.742715 & 0.009009 \\
\hline $\mathrm{C}$ & -0.597581 & -7.046369 & 0.079770 \\
\hline $\mathrm{C}$ & 0.628956 & -9.148235 & -0.139591 \\
\hline $\mathrm{C}$ & -0.583315 & -9.844208 & -0.218033 \\
\hline $\mathrm{C}$ & -1.860887 & 9.200638 & -0.280514 \\
\hline $\mathrm{C}$ & -3.083736 & 9.884223 & -0.307833 \\
\hline $\mathrm{C}$ & -1.861115 & 7.715955 & -0.186681 \\
\hline $\mathrm{C}$ & -3.040023 & 7.037233 & -0.136002 \\
\hline C & -1.846961 & 4.925656 & 0.035450 \\
\hline $\mathrm{C}$ & -3.062595 & 5.632349 & -0.031099 \\
\hline $\mathrm{C}$ & -1.860142 & 3.528510 & 0.177355 \\
\hline C & -3.083678 & 2.822516 & 0.229421 \\
\hline C & -1.817628 & 0.687298 & 0.535904 \\
\hline $\mathrm{C}$ & -3.078330 & 1.435796 & 0.353063 \\
\hline $\mathrm{C}$ & -1.841978 & -0.667694 & 0.632764 \\
\hline $\mathrm{C}$ & -3.112023 & -1.405065 & 0.472551 \\
\hline $\mathrm{C}$ & -1.870410 & -3.508777 & 0.440974 \\
\hline $\mathrm{C}$ & -3.063240 & -2.833083 & 0.400142 \\
\hline C & -1.806941 & -4.904723 & 0.286442 \\
\hline $\mathrm{C}$ & -3.081825 & -5.662849 & 0.141510 \\
\hline $\mathrm{C}$ & -1.816612 & -7.752232 & -0.022433 \\
\hline C & -3.050188 & -7.062927 & 0.004032 \\
\hline C & -1.802272 & -9.157924 & -0.168937 \\
\hline $\mathrm{C}$ & -3.007006 & -9.863166 & -0.275531 \\
\hline $\mathrm{C}$ & -4.304409 & 9.189263 & -0.254070 \\
\hline $\mathrm{C}$ & -5.512353 & 9.893714 & -0.272708 \\
\hline $\mathrm{C}$ & -4.324709 & 7.779966 & -0.176346 \\
\hline $\mathrm{C}$ & -5.556990 & 7.080396 & -0.130889 \\
\hline $\mathrm{C}$ & -4.290370 & 4.930457 & 0.013011 \\
\hline $\mathrm{C}$ & -5.580825 & 5.669232 & -0.052316 \\
\hline $\mathrm{C}$ & -4.293012 & 3.528917 & 0.126784 \\
\hline $\mathrm{C}$ & -5.573285 & 2.788605 & 0.131978 \\
\hline $\mathrm{C}$ & -4.303801 & 0.698555 & 0.322310 \\
\hline C & -5.576685 & 1.438564 & 0.200559 \\
\hline $\mathrm{C}$ & -4.310317 & -0.712993 & 0.359980 \\
\hline $\mathrm{C}$ & -5.594856 & -1.452573 & 0.227990 \\
\hline $\mathrm{C}$ & -4.343400 & -3.571826 & 0.233121 \\
\hline $\mathrm{C}$ & -5.573633 & -2.869192 & 0.174179 \\
\hline
\end{tabular}




\begin{tabular}{|c|c|c|c|}
\hline $\mathrm{C}$ & -4.318030 & -4.975034 & 0.126166 \\
\hline C & -5.527353 & -5.690379 & -0.010411 \\
\hline $\mathrm{C}$ & -4.259368 & -7.780147 & -0.123387 \\
\hline $\mathrm{C}$ & -5.496774 & -7.095393 & -0.126641 \\
\hline $\mathrm{C}$ & -4.231498 & -9.185130 & -0.256616 \\
\hline $\mathrm{C}$ & -5.428850 & -9.896629 & -0.377911 \\
\hline C & -6.737267 & 9.213922 & -0.218510 \\
\hline $\mathrm{C}$ & -7.929935 & 9.938374 & -0.223859 \\
\hline C & -6.767855 & 7.804068 & -0.155810 \\
\hline $\mathrm{C}$ & -8.006536 & 7.125146 & -0.111116 \\
\hline $\mathrm{C}$ & -6.818078 & 4.968499 & -0.027319 \\
\hline $\mathrm{C}$ & -8.031313 & 5.702039 & -0.065890 \\
\hline $\mathrm{C}$ & -6.842926 & 3.547046 & 0.046866 \\
\hline C & -8.057967 & 2.827029 & 0.031407 \\
\hline $\mathrm{C}$ & -6.846882 & 0.679228 & 0.148447 \\
\hline C & -8.059388 & 1.400506 & 0.066771 \\
\hline $\mathrm{C}$ & -6.824151 & -0.744934 & 0.146259 \\
\hline $\mathrm{C}$ & -8.033270 & -1.474544 & 0.062948 \\
\hline C & -6.784625 & -3.589057 & 0.050148 \\
\hline $\mathrm{C}$ & -8.014649 & -2.896501 & 0.017634 \\
\hline $\mathrm{C}$ & -6.763628 & -5.001950 & -0.034770 \\
\hline $\mathrm{C}$ & -7.973814 & -5.726934 & -0.160395 \\
\hline $\mathrm{C}$ & -6.699948 & -7.820735 & -0.257612 \\
\hline $\mathrm{C}$ & -7.940974 & -7.146132 & -0.273072 \\
\hline $\mathrm{C}$ & -6.658046 & -9.225532 & -0.378671 \\
\hline $\mathrm{C}$ & -7.842871 & -9.951744 & -0.502892 \\
\hline $\mathrm{C}$ & -9.173995 & 9.295502 & -0.165282 \\
\hline $\mathrm{C}$ & -10.314080 & 10.113753 & -0.171307 \\
\hline $\mathrm{C}$ & -9.220474 & 7.870427 & -0.107479 \\
\hline $\mathrm{C}$ & -10.433785 & 7.162467 & -0.032662 \\
\hline C & -9.270953 & 5.021198 & -0.043343 \\
\hline $\mathrm{C}$ & -10.457079 & 5.774489 & 0.003424 \\
\hline $\mathrm{C}$ & -9.282932 & 3.523124 & -0.047083 \\
\hline $\mathrm{C}$ & -10.474658 & 2.781214 & -0.162463 \\
\hline $\mathrm{C}$ & -9.284589 & 0.704362 & -0.000751 \\
\hline $\mathrm{C}$ & -10.475360 & 1.444838 & -0.142931 \\
\hline $\mathrm{C}$ & -9.271432 & -0.793384 & 0.02 \\
\hline $\mathrm{C}$ & -10.457190 & -1.548776 & 0.044376 \\
\hline $\mathrm{C}$ & -9.236054 & -3.616877 & -0.060088 \\
\hline $\mathrm{C}$ & -10.442197 & -2.882764 & 0.001496 \\
\hline $\mathrm{C}$ & -9.214630 & -5.039058 & -0.178770 \\
\hline $\mathrm{C}$ & -10.392406 & -5.800202 & -0.342958 \\
\hline $\mathrm{C}$ & -9.145708 & -7.890740 & -0.409825 \\
\hline $\mathrm{C}$ & -10.358782 & -7.184140 & -0.449352 \\
\hline C & -9.090097 & -9.312921 & -0.516399 \\
\hline $\mathrm{C}$ & -10.223698 & -10.132131 & -0.632743 \\
\hline $\mathrm{H}$ & 11.224156 & 10.008875 & -0.255490 \\
\hline $\mathrm{H}$ & 9.050103 & 11.14 & 2095 \\
\hline $\mathrm{H}$ & 11.374792 & 7.603513 & -0.161335 \\
\hline $\mathrm{H}$ & 11.416246 & 5.516238 & -0.087097 \\
\hline $\mathrm{H}$ & 11.457017 & 3.221322 & -0 . \\
\hline $\mathrm{H}$ & 11.456166 & 1.147795 & -0.290858 \\
\hline $\mathrm{H}$ & 11.449411 & -1.143394 & -0.295366 \\
\hline $\mathrm{H}$ & 11.441500 & -3.206454 & -0.077835 \\
\hline $\mathrm{H}$ & 11.414078 & -5.479246 & -0.184008 \\
\hline $\mathrm{H}$ & 11.378597 & -7.524537 & -0.434494 \\
\hline $\mathrm{H}$ & 11.231292 & -9.911163 & -0.600830 \\
\hline $\mathrm{H}$ & 9.067 & -11.060 & -0.5 \\
\hline $\mathrm{H}$ & 6.634314 & 11.049 & 6361 \\
\hline $\mathrm{H}$ & 6.652123 & -10.988118 & -0.462541 \\
\hline $\mathrm{H}$ & 4.23 & 11.003 & -0.3 \\
\hline $\mathrm{H}$ & 4.244679 & -10.945562 & -0.376421 \\
\hline $\mathrm{H}$ & 1.800203 & 10.993879 & -0.394712 \\
\hline $\mathrm{H}$ & 1.834140 & -10.923768 & -0.329932 \\
\hline $\mathrm{H}$ & -0.648579 & 10.982414 & -0.395750 \\
\hline $\mathrm{H}$ & -0.577959 & -10.923184 & -0.332863 \\
\hline $\mathrm{H}$ & -3.086562 & 10.967260 & -0.369519 \\
\hline $\mathrm{H}$ & -2.991326 & -10.942590 & -0.385022 \\
\hline $\mathrm{H}$ & -5.499711 & 10.977423 & -0 . \\
\hline $\mathrm{H}$ & -5.403 & -10.97693 & -0 . \\
\hline $\mathrm{H}$ & & 11.0 & \\
\hline
\end{tabular}




$$
\begin{array}{r}
-7.790763 \\
-11.325192 \\
-10.219622 \\
-11.391096 \\
-11.428135 \\
-11.431479 \\
-11.433590 \\
-11.427125 \\
-11.407808 \\
-11.370729 \\
-11.309644 \\
-11.237016 \\
-10.121904 \\
0.716411 \\
0.000208
\end{array}
$$

$$
\begin{array}{r}
-11.032835 \\
9.744609 \\
11.192526 \\
7.655421 \\
5.315254 \\
3.259347 \\
0.966750 \\
-1.083475 \\
-3.357177 \\
-5.358847 \\
-7.674674 \\
-9.767663 \\
-11.208018 \\
-0.658955 \\
-0.085679
\end{array}
$$

$$
\begin{array}{r}
-0.589525 \\
-0.140323 \\
-0.213401 \\
0.004883 \\
0.084629 \\
-0.293233 \\
-0.259318 \\
0.101906 \\
0.032821 \\
-0.418917 \\
-0.577579 \\
-0.637272 \\
-0.714103 \\
2.507425 \\
2.838966
\end{array}
$$

TS1

C $\quad-11.306521$

C $\quad-10.181470$

$-11.185609$

$-9.919648$

$-10.854795$

$-9.757739$

$-10.697880$

$-9.424918$

$-10.311067$

$-9.241009$

$-10.130151$

$-8.857189$

$-9.743337$

$-8.665279$

$-9.557422$

$-8.269458$

$-9.128608$

$-8.063548$

$-8.886695$

$-7.588726$

$-8.913505$

$-7.787452$

$-8.773595$

$-7.485630$

$-8.456774$

$-7.327719$

$-8.300863$

$-6.952901$

$-7.942044$

$-6.784986$

$-7.740134$

$-6.438168$

$-7.352313$

$-6.242258$

$-7.153403$

$-5.843436$

$-6.737581$

$-5.636525$

$-6.508670$

$-5.204702$

$-6.507486$

$-5.386433$

$-6.354575$

$-4.992611$

$-5.961755$

$-4.838700$

$-5.785843$

$-4.502877$

$-5.434022$

$-4.330966$

$-5.261111$

$-3.971146$

$-4.938292$
$-7.663681$

$-8.476609$

$-6.282673$

$-5.669593$

$-3.365652$

$-4.256582$

$-2.039642$

$-1.438561$

0.891383

$-0.015549$

2. 216394

2.803475

5.131419

4.219487

6.454431

7.031513

9.371458

8.442526

10.741473

11.232556

$-7.915528$

$-8.747457$

$-6.511519$

$-5.961437$

$-3.708499$

$-4.565427$

$-2.298489$

$-1.717403$

0.528534

$-0.384393$

1. 937546

2. 482629

4. 750571

3.883642

6.158584

6.686667

8.954610

8.079681

10.352625

10.858708

$-8.210375$

$-9.055303$

$-6.815365$

$-6.251278$

$-4.005340$

$-4.859269$

$-2.618329$

$-2.078247$

0.183694

$-0.685236$

1. 582705

2.132787
4. 426725
$-1.167983$

$-1.021983$

$-0.830983$

$-0.676983$

$-0.681983$

$-0.577983$

$-0.471983$

$-0.579983$

$-0.425983$

$-0.550983$

$-0.360983$

$-0.370983$

$-0.332983$

$-0.404983$

$-0.402983$

$-0.618983$

$-0.484983$

$-0.699983$

$-0.656983$

$-0.957983$

$-0.940983$

$-0.803983$

$-0.630983$

$-0.523983$

$-0.486983$

$-0.410983$

$-0.228983$

$-0.258983$

$-0.141983$

$-0.231983$

$-0.094983$

$-0.256983$

$-0.131983$

$-0.302983$

$-0.253983$

$-0.448983$

$-0.330983$

$-0.535983$

$-0.507983$

$-0.774983$

$-0.780983$

$-0.437983$

$-0.298983$

$-0.274983$

$-0.153983$

0.046017

0.055017

0.180017

0.074017

0.220017

$-0.045983$
$-1.135983$

$-0.613983$ 


\begin{tabular}{|c|c|c|c|}
\hline C & -3.765235 & 3.528800 & 0.133017 \\
\hline $\mathrm{C}$ & -4.737381 & 5.819738 & -0.126983 \\
\hline $\mathrm{C}$ & -3.426415 & 6.346822 & -0.087983 \\
\hline C & -4.320559 & 8.602765 & -0.302983 \\
\hline $\mathrm{C}$ & -3.214503 & 7.733835 & -0.188983 \\
\hline $\mathrm{C}$ & -4.110647 & 9.995778 & -0.396983 \\
\hline C & -2.807680 & 10.511861 & -0.388983 \\
\hline $\mathrm{C}$ & -4.092465 & -8.539221 & -0.633983 \\
\hline $\mathrm{C}$ & -2.971412 & -9.385149 & -0.676983 \\
\hline $\mathrm{C}$ & -3.911559 & -7.074209 & -0.446983 \\
\hline $\mathrm{C}$ & -2.609592 & -6.555126 & -0.296983 \\
\hline $\mathrm{C}$ & -3.550735 & -4.320186 & -0.098983 \\
\hline $\mathrm{C}$ & -2.430680 & -5.174115 & -0.108983 \\
\hline $\mathrm{C}$ & -3.386823 & -2.934176 & 0.078017 \\
\hline $\mathrm{C}$ & -2.066857 & -2.396092 & 0.297017 \\
\hline C & -3.011001 & -0.138152 & 0.415017 \\
\hline $\mathrm{C}$ & -1.912945 & -1.026082 & 0.534017 \\
\hline $\mathrm{C}$ & -2.847089 & 1.244858 & 0.433017 \\
\hline C & -1.573124 & 1.786940 & 0.565017 \\
\hline C & -2.507268 & 4.044880 & 0.183017 \\
\hline $\mathrm{C}$ & -1.352210 & 3.143954 & 0.378017 \\
\hline $\mathrm{C}$ & -2.263356 & 5.429896 & 0.050017 \\
\hline $\mathrm{C}$ & -0.993388 & 5.934977 & 0.035017 \\
\hline $\mathrm{C}$ & -1.902537 & 8.259919 & -0.188983 \\
\hline $\mathrm{C}$ & -0.786482 & 7.400990 & -0.098983 \\
\hline $\mathrm{C}$ & -1.703626 & 9.654931 & -0.294983 \\
\hline $\mathrm{C}$ & -0.403659 & 10.179014 & -0.314983 \\
\hline $\mathrm{C}$ & -1.672445 & -8.866067 & -0.554983 \\
\hline $\mathrm{C}$ & -0.547391 & -9.701995 & -0.633983 \\
\hline $\mathrm{C}$ & -1.486538 & -7.407055 & -0.353983 \\
\hline C & -0.185572 & -6.871972 & -0.250983 \\
\hline $\mathrm{C}$ & -1.134714 & -4.649032 & 0.037017 \\
\hline $\mathrm{C}$ & -0.011660 & -5.489961 & -0.057983 \\
\hline $\mathrm{C}$ & -0.955804 & -3.238021 & 0.260017 \\
\hline $\mathrm{C}$ & 0.334163 & -2.708939 & 0.350017 \\
\hline $\mathrm{C}$ & -0.618974 & -0.556999 & 0.972017 \\
\hline $\mathrm{C}$ & 0.526076 & -1.350926 & 0.603017 \\
\hline $\mathrm{C}$ & -0.416072 & 0.970014 & 8017 \\
\hline $\mathrm{C}$ & 0.917897 & 1.455099 & 017 \\
\hline $\mathrm{C}$ & -0.056243 & 3.648036 & 0.323017 \\
\hline $\mathrm{C}$ & 1.070811 & 2.812108 & 0.375017 \\
\hline C & 0.141665 & 5.094049 & 0.131017 \\
\hline $\mathrm{C}$ & 1.458632 & 5.607133 & 0.050017 \\
\hline $\mathrm{C}$ & 0.521484 & 7.932073 & -0.137983 \\
\hline C & 1.645539 & 7.077145 & -0.079983 \\
\hline $\mathrm{C}$ & 0.708395 & 9.328085 & -0.245983 \\
\hline $\mathrm{C}$ & 2.004361 & 9.859168 & -0.290983 \\
\hline $\mathrm{C}$ & 0.751575 & -9.171912 & -0.547983 \\
\hline $\mathrm{C}$ & 1.874628 & -10.002840 & -0.651983 \\
\hline $\mathrm{C}$ & 0.944482 & 708900 & -0.358983 \\
\hline $\mathrm{C}$ & 2.199448 & -7.18 & -0.306983 \\
\hline $\mathrm{C}$ & 1.290306 & -4.952878 & 0.002017 \\
\hline $\mathrm{C}$ & 2.402360 & -5.805807 & -0.136983 \\
\hline $\mathrm{C}$ & 1.481218 & -3.570866 & 0.180017 \\
\hline C & 2.781183 & -3.031783 & 0.189017 \\
\hline $\mathrm{C}$ & 1.827039 & -0.773843 & 0.504017 \\
\hline $\mathrm{C}$ & 2.969095 & -1.640771 & 0.308017 \\
\hline C & 2.010952 & 0.591168 & 0.491017 \\
\hline $\mathrm{C}$ & 3.359915 & 1.172254 & 0.293017 \\
\hline $\mathrm{C}$ & 2.426774 & 3.387195 & 0.208017 \\
\hline $\mathrm{C}$ & 3.514826 & 2.569264 & 0.190017 \\
\hline $\mathrm{C}$ & 2.553685 & 4.789203 & 0.080017 \\
\hline $\mathrm{C}$ & 3.918647 & 5.372290 & -0.026983 \\
\hline $\mathrm{C}$ & 2.948504 & 7.617228 & -0.146983 \\
\hline $\mathrm{C}$ & 4.077558 & 6.767300 & -0.123983 \\
\hline $\mathrm{C}$ & 3.123415 & 9.015239 & -0.247983 \\
\hline $\mathrm{C}$ & 4.414381 & 9.555322 & -0.309983 \\
\hline $\mathrm{C}$ & 3.175594 & -9.472757 & -0.601983 \\
\hline $\mathrm{C}$ & 4.282648 & -10.318687 & 3983 \\
\hline $\mathrm{C}$ & 506 & 745 & -0 \\
\hline $\mathrm{C}$ & & & \\
\hline
\end{tabular}




\begin{tabular}{|c|c|c|c|}
\hline $\mathrm{C}$ & 3.706326 & -5.268724 & -0.121983 \\
\hline C & 4.889383 & -6.159648 & -0.274983 \\
\hline $\mathrm{C}$ & 3.888238 & -3.883712 & 0.022017 \\
\hline C & 5.250201 & -3.315625 & -0.026983 \\
\hline $\mathrm{C}$ & 4.264059 & -1.086688 & 0.198017 \\
\hline C & 5.428116 & -1.983614 & 0.043017 \\
\hline $\mathrm{C}$ & 4.462970 & 0.307325 & 0.196017 \\
\hline C & 5.834935 & 0.868412 & 0.057017 \\
\hline $\mathrm{C}$ & 4.883790 & 3.131351 & 0.050017 \\
\hline $\mathrm{C}$ & 6.007845 & 2.271423 & 0.007017 \\
\hline C & 5.050701 & 4.525362 & -0.034983 \\
\hline $\mathrm{C}$ & 6.346666 & 5.073445 & -0.129983 \\
\hline $\mathrm{C}$ & 5.374523 & 7.318383 & -0.203983 \\
\hline $\mathrm{C}$ & 6.508577 & 6.472455 & -0.202983 \\
\hline $\mathrm{C}$ & 5.538434 & 8.717393 & -0.288983 \\
\hline $\mathrm{C}$ & 6.824399 & 9.263475 & -0.355983 \\
\hline $\mathrm{C}$ & 5.584615 & -9.798604 & -0.699983 \\
\hline $\mathrm{C}$ & 6.675670 & -10.659534 & -0.827983 \\
\hline C & 5.795526 & -8.410590 & -0.553983 \\
\hline $\mathrm{C}$ & 7.111493 & -7.889507 & -0.546983 \\
\hline $\mathrm{C}$ & 6.203348 & -5.621564 & -0.279983 \\
\hline $\mathrm{C}$ & 7.317404 & -6.485493 & -0.411983 \\
\hline $\mathrm{C}$ & 6.409259 & -4.225551 & -0.170983 \\
\hline C & 7.709225 & -3.679468 & -0.194983 \\
\hline $\mathrm{C}$ & 6.786079 & -1.401527 & -0.038983 \\
\hline $\mathrm{C}$ & 7.897134 & -2.265456 & -0.135983 \\
\hline $\mathrm{C}$ & 6.954990 & 0.004484 & -0.027983 \\
\hline $\mathrm{C}$ & 8.253955 & 0.551566 & -0.124983 \\
\hline C & 7.306810 & 2.819506 & -0.094983 \\
\hline C & 8.430865 & 1.964578 & -0.149983 \\
\hline $\mathrm{C}$ & 7.478721 & 4.223517 & -0.150983 \\
\hline $\mathrm{C}$ & 8.777685 & 4.780600 & -0.226983 \\
\hline $\mathrm{C}$ & 7.801542 & 7.030538 & -0.276983 \\
\hline $\mathrm{C}$ & 8.940595 & 6.193610 & -0.278983 \\
\hline $\mathrm{C}$ & 7.952452 & 8.431547 & -0.346983 \\
\hline $\mathrm{C}$ & 9.228417 & 8.992629 & -0.410983 \\
\hline $\mathrm{C}$ & 7.990639 & -10.175450 & -0.819983 \\
\hline $\mathrm{C}$ & 9.017699 & -11.123385 & -0.946983 \\
\hline $\mathrm{C}$ & 8.217549 & -8.772436 & -0.684983 \\
\hline $\mathrm{C}$ & 9.509514 & -8.219354 & -0.695983 \\
\hline C & 8.634370 & -5.951409 & -0.413983 \\
\hline $\mathrm{C}$ & 9.711427 & -6.851341 & -0.568983 \\
\hline $\mathrm{C}$ & 8.831280 & -4.540397 & -0.287983 \\
\hline $\mathrm{C}$ & 10.115242 & -3.952315 & -0.239983 \\
\hline $\mathrm{C}$ & 9.205100 & -1.723373 & -0.181983 \\
\hline C & 10.291158 & -2.627304 & -0.189983 \\
\hline $\mathrm{C}$ & 9.383009 & -0.305362 & -0.207983 \\
\hline $\mathrm{C}$ & 10.653971 & 0.291719 & -0.354983 \\
\hline $\mathrm{C}$ & 9.735830 & 2.511661 & -0.246983 \\
\hline $\mathrm{C}$ & 10.819887 & 1.616730 & -0.371983 \\
\hline $\mathrm{C}$ & 9.912739 & 3.929672 & -0.246983 \\
\hline C & 11.190701 & 4.530754 & -0.255983 \\
\hline $\mathrm{C}$ & 10.238558 & 6.771693 & -0.333983 \\
\hline $\mathrm{C}$ & 11.347613 & 5.909764 & -0.301983 \\
\hline C & 10.378468 & 8.190702 & -0.404983 \\
\hline C & 11.614426 & 8.849781 & -0.480983 \\
\hline $\mathrm{H}$ & -12.284493 & -8.106743 & -1.312983 \\
\hline $\mathrm{H}$ & -10.303401 & -9.546617 & -1.255983 \\
\hline $\mathrm{H}$ & -12.106645 & -5.728732 & -1.072983 \\
\hline $\mathrm{H}$ & -11.872774 & -3.707717 & -0.748983 \\
\hline $\mathrm{H}$ & -11.607917 & -1.463700 & -0.570983 \\
\hline $\mathrm{H}$ & -11.321046 & 0.564318 & -0.757983 \\
\hline $\mathrm{H}$ & -11.016189 & 2.805338 & -0.713983 \\
\hline $\mathrm{H}$ & -10.770317 & 4.810353 & -0.353983 \\
\hline $\mathrm{H}$ & -10.453460 & 7.050373 & -0.425983 \\
\hline $\mathrm{H}$ & -10.161589 & 9.077392 & -0.674983 \\
\hline $\mathrm{H}$ & -9.716739 & 11.430420 & -0.801983 \\
\hline $\mathrm{H}$ & -7.427795 & 12.302566 & -0.725983 \\
\hline $\mathrm{H}$ & -7.907384 & -9.818464 & -1.064983 \\
\hline $\mathrm{H}$ & -5.039771 & 11.928719 & -0.579983 \\
\hline $\mathrm{H}$ & -5.520364 & -10.123312 & -0.914983 \\
\hline
\end{tabular}




\begin{tabular}{|c|c|c|c|}
\hline $\mathrm{C}$ & -7.405934 & -6.082853 & -0.701969 \\
\hline $\mathrm{C}$ & -8.412749 & -3.834725 & -0.569420 \\
\hline $\mathrm{C}$ & -7.273602 & -4.679628 & -0.546514 \\
\hline $\mathrm{C}$ & -8.279357 & -2.425868 & -0.438830 \\
\hline $\mathrm{C}$ & -6.943378 & -1.814684 & -0.252418 \\
\hline $\mathrm{C}$ & -7.956271 & 0.435905 & -0.239925 \\
\hline $\mathrm{C}$ & -6.792650 & -0.475082 & -0.138337 \\
\hline $\mathrm{C}$ & -7.768861 & 1.844490 & -0.205608 \\
\hline $\mathrm{C}$ & -6.473088 & 2.399681 & -0.065338 \\
\hline $\mathrm{C}$ & -7.412351 & 4.656915 & -0.226285 \\
\hline $\mathrm{C}$ & -6.292173 & 3.802688 & -0.104620 \\
\hline $\mathrm{C}$ & -7.231179 & 6.066174 & -0.273800 \\
\hline $\mathrm{C}$ & -5.927792 & 6.607318 & -0.236614 \\
\hline $\mathrm{C}$ & -6.851725 & 8.864454 & -0.432716 \\
\hline $\mathrm{C}$ & -5.738765 & 8.002128 & -0.321574 \\
\hline $\mathrm{C}$ & -6.640768 & 10.264029 & -0.528872 \\
\hline $\mathrm{C}$ & -5.342184 & 10.784164 & -0.520901 \\
\hline $\mathrm{C}$ & -6.380808 & -8.311177 & -0.859229 \\
\hline $\mathrm{C}$ & -5.244973 & -9.134541 & -0.861002 \\
\hline $\mathrm{C}$ & -6.257191 & -6.913624 & -0.687565 \\
\hline $\mathrm{C}$ & -4.906229 & -6.323269 & -0.499426 \\
\hline C & -5.915308 & -4.093394 & -0.351187 \\
\hline $\mathrm{C}$ & -4.777251 & -4.929617 & -0.327406 \\
\hline $\mathrm{C}$ & -5.762488 & -2.701059 & -0.192374 \\
\hline $\mathrm{C}$ & -4.490509 & -2.143704 & 0.025903 \\
\hline $\mathrm{C}$ & -5.446443 & 0.107759 & 0.072757 \\
\hline $\mathrm{C}$ & -4.318846 & -0.797064 & 0.202351 \\
\hline $\mathrm{C}$ & -5.287574 & 1.510839 & 0.106437 \\
\hline $\mathrm{C}$ & -4.017615 & 2.058846 & 0.297924 \\
\hline $\mathrm{C}$ & -4.993424 & 4.358788 & -0.023611 \\
\hline $\mathrm{C}$ & -3.809987 & 3.474583 & 0.164628 \\
\hline $\mathrm{C}$ & -4.809623 & 5.753162 & -0.110875 \\
\hline $\mathrm{C}$ & -3.505804 & 6.294128 & -0.076585 \\
\hline C & -4.430405 & 8.539303 & -0.307107 \\
\hline $\mathrm{C}$ & -3.313134 & 7.684109 & -0.190763 \\
\hline $\mathrm{C}$ & -4.237950 & 9.934062 & -0.415370 \\
\hline $\mathrm{C}$ & -2.942601 & 10.464616 & -0.425493 \\
\hline $\mathrm{C}$ & -3.965303 & -8.596455 & -0.697652 \\
\hline $\mathrm{C}$ & -2.833128 & -9.425840 & -0.728066 \\
\hline $\mathrm{C}$ & -3.810561 & -7.128950 & -0.503629 \\
\hline $\mathrm{C}$ & -2.518372 & -6.591024 & -0.337852 \\
\hline $\mathrm{C}$ & -3.498561 & -4.369304 & -0.132825 \\
\hline $\mathrm{C}$ & -2.361926 & -5.207807 & -0.138412 \\
\hline $\mathrm{C}$ & -3.318343 & -3.019796 & 0.072815 \\
\hline $\mathrm{C}$ & -1.993582 & -2.432209 & 0.341349 \\
\hline $\mathrm{C}$ & -2.991137 & -0.212506 & 0.489389 \\
\hline $\mathrm{C}$ & -1.894884 & -1.073324 & 0.625941 \\
\hline $\mathrm{C}$ & -2.850333 & 1.175806 & 0.535262 \\
\hline $\mathrm{C}$ & -1.597406 & 1.758051 & 0.690071 \\
\hline $\mathrm{C}$ & -2.593290 & 3.979322 & 0.265973 \\
\hline $\mathrm{C}$ & -1.409576 & 3.113932 & 0.469387 \\
\hline $\mathrm{C}$ & -2.330137 & 5.392182 & 0.075424 \\
\hline $\mathrm{C}$ & -1.067674 & 5.915329 & 0.059678 \\
\hline $\mathrm{C}$ & -2.008751 & 8.226149 & -0.201951 \\
\hline $\mathrm{C}$ & -0.882666 & 7.381907 & -0.101393 \\
\hline $\mathrm{C}$ & -1.829056 & 9.622193 & -0.329139 \\
\hline $\mathrm{C}$ & -0.537494 & 10.161493 & -0.367314 \\
\hline $\mathrm{C}$ & -1.546058 & -8.890290 & -0.587681 \\
\hline $\mathrm{C}$ & -0.411395 & -9.712756 & -0.649295 \\
\hline $\mathrm{C}$ & -1.382164 & -7.428684 & -0.383028 \\
\hline $\mathrm{C}$ & -0.089071 & -6.879050 & -0.261804 \\
\hline $\mathrm{C}$ & -1.004422 & -4.683059 & 0.044128 \\
\hline $\mathrm{C}$ & 0.065256 & -5.496746 & -0.058595 \\
\hline $\mathrm{C}$ & -0.860279 & -3.245476 & 0.306243 \\
\hline $\mathrm{C}$ & 0.416589 & -2.698394 & 0.425706 \\
\hline $\mathrm{C}$ & -0.599583 & -0.585543 & 1.200356 \\
\hline $\mathrm{C}$ & 0.595917 & -1.349601 & 0.716601 \\
\hline C & -0.415347 & 0.988061 & 1.223987 \\
\hline $\mathrm{C}$ & 0.896938 & 1.476434 & 0.655373 \\
\hline $\mathrm{C}$ & -0.125482 & 3.655203 & 0.396019 \\
\hline $\mathrm{C}$ & 1.011596 & 2.842000 & 0.437002 \\
\hline
\end{tabular}




\begin{tabular}{|c|c|c|c|}
\hline $\mathrm{C}$ & 0.032505 & 5.100814 & 0.156742 \\
\hline $\mathrm{C}$ & 1.386792 & 5.622382 & 0.075089 \\
\hline $\mathrm{C}$ & 0.417094 & 7.929911 & -0.155805 \\
\hline C & 1.551309 & 7.091701 & -0.087859 \\
\hline $\mathrm{C}$ & 0.584011 & 9.326764 & -0.291433 \\
\hline $\mathrm{C}$ & 1.870801 & 9.874387 & -0.356594 \\
\hline $\mathrm{C}$ & 0.878521 & -9.169084 & -0.545255 \\
\hline $\mathrm{C}$ & 2.010364 & -9.989732 & -0.627699 \\
\hline $\mathrm{C}$ & 1.052397 & -7.703461 & -0.357228 \\
\hline $\mathrm{C}$ & 2.301406 & -7.172163 & -0.293186 \\
\hline $\mathrm{C}$ & 1.363471 & -4.947521 & 0.018999 \\
\hline $\mathrm{C}$ & 2.487469 & -5.787108 & -0.122204 \\
\hline $\mathrm{C}$ & 1.597882 & -3.559855 & 0.235177 \\
\hline $\mathrm{C}$ & 2.835899 & -3.014509 & 0.214830 \\
\hline $\mathrm{C}$ & 1.850255 & -0.741792 & 0.577447 \\
\hline $\mathrm{C}$ & 3.027190 & -1.593599 & 0.309200 \\
\hline $\mathrm{C}$ & 2.001021 & 0.640481 & 0.554159 \\
\hline $\mathrm{C}$ & 3.336321 & 1.244246 & 0.303947 \\
\hline $\mathrm{C}$ & 2.356448 & 3.428928 & 0.219534 \\
\hline $\mathrm{C}$ & 3.478437 & 2.608437 & 0.239215 \\
\hline $\mathrm{C}$ & 2.490408 & 4.817672 & 0.109606 \\
\hline $\mathrm{C}$ & 3.845162 & 5.418275 & -0.024356 \\
\hline $\mathrm{C}$ & 2.844364 & 7.647623 & -0.175930 \\
\hline $\mathrm{C}$ & 3.984108 & 6.814026 & -0.147441 \\
\hline $\mathrm{C}$ & 2.999045 & 9.046639 & -0.305938 \\
\hline $\mathrm{C}$ & 4.280537 & 9.603111 & -0.390632 \\
\hline $\mathrm{C}$ & 3.303592 & -9.446286 & -0.564634 \\
\hline $\mathrm{C}$ & 4.419331 & -10.283145 & -0.663938 \\
\hline $\mathrm{C}$ & 3.489365 & -8.055517 & -0.413410 \\
\hline $\mathrm{C}$ & 4.794537 & -7.505631 & -0.385165 \\
\hline $\mathrm{C}$ & 3.787937 & -5.234886 & -0.105320 \\
\hline $\mathrm{C}$ & 4.982820 & -6.112266 & -0.251356 \\
\hline C & 3.952738 & -3.848710 & 0.037796 \\
\hline $\mathrm{C}$ & 5.305959 & -3.259438 & -0.012484 \\
\hline $\mathrm{C}$ & 4.283173 & -1.044113 & 0.235750 \\
\hline $\mathrm{C}$ & 5.461196 & -1.921912 & 0.062103 \\
\hline $\mathrm{C}$ & 4.455834 & 0.356199 & 0.239918 \\
\hline $\mathrm{C}$ & 5.819853 & 0.939409 & 0.078782 \\
\hline $\mathrm{C}$ & 4.838637 & 3.189647 & 0.071192 \\
\hline $\mathrm{C}$ & 5.973115 & 2.345790 & 0.019292 \\
\hline $\mathrm{C}$ & 4.986116 & 4.586284 & -0.033776 \\
\hline $\mathrm{C}$ & 6.273367 & 5.151999 & -0.153759 \\
\hline $\mathrm{C}$ & 5.270823 & 7.381911 & -0.252035 \\
\hline $\mathrm{C}$ & 6.415163 & 6.552373 & -0.249387 \\
\hline $\mathrm{C}$ & 5.414134 & 8.782015 & -0.364340 \\
\hline $\mathrm{C}$ & 6.690211 & 9.345422 & -0.453265 \\
\hline $\mathrm{C}$ & 5.715618 & -9.751532 & -0.631679 \\
\hline $\mathrm{C}$ & 6.814665 & -10.605035 & -0.734577 \\
\hline $\mathrm{C}$ & 5.911615 & -8.359806 & -0.500753 \\
\hline $\mathrm{C}$ & 7.220791 & -7.828484 & -0.490696 \\
\hline $\mathrm{C}$ & 6.292434 & -5.558742 & -0.255553 \\
\hline $\mathrm{C}$ & 7.411781 & -6.422173 & -0.372663 \\
\hline $\mathrm{C}$ & 6.480341 & -4.152639 & -0.155517 \\
\hline $\mathrm{C}$ & 7.769720 & -3.574989 & -0.182771 \\
\hline C & 6.807484 & -1.314905 & -0.028802 \\
\hline C & 7.932832 & -2.158908 & -0.131374 \\
\hline $\mathrm{C}$ & 6.953446 & 0.093329 & -0.020766 \\
\hline $\mathrm{C}$ & 8.242623 & 0.661742 & -0.137596 \\
\hline $\mathrm{C}$ & 7.263384 & 2.913427 & -0.106893 \\
\hline $\mathrm{C}$ & 8.398190 & 2.075656 & -0.172973 \\
\hline $\mathrm{C}$ & 7.415501 & 4.319162 & -0.179501 \\
\hline $\mathrm{C}$ & 8.705657 & 4.894278 & -0.277094 \\
\hline $\mathrm{C}$ & 7.698601 & 7.129086 & -0.345615 \\
\hline $\mathrm{C}$ & 8.848406 & 6.309531 & -0.346874 \\
\hline $\mathrm{C}$ & 7.829137 & 8.530684 & -0.439154 \\
\hline $\mathrm{C}$ & 9.095360 & 9.110179 & -0.521789 \\
\hline $\mathrm{C}$ & 8.125585 & -10.110159 & -0.720425 \\
\hline C & 9.162372 & -11.050998 & -0.817855 \\
\hline $\mathrm{C}$ & 8.338449 & -8.703673 & -0.608270 \\
\hline $\mathrm{C}$ & 9.625749 & -8.138225 & -0.619972 \\
\hline $\mathrm{C}$ & 8.721997 & -5.887557 & -0.377343 \\
\hline
\end{tabular}




\begin{tabular}{|c|c|c|c|}
\hline $\mathrm{C}$ & 9.811396 & -6.766753 & -0.513061 \\
\hline $\mathrm{C}$ & 8.907318 & -4.404177 & -0.266273 \\
\hline $\mathrm{C}$ & 10.180580 & -3.805045 & -0.228390 \\
\hline $\mathrm{C}$ & 9.231507 & -1.595285 & -0.189587 \\
\hline $\mathrm{C}$ & 10.335062 & -2.478410 & -0.191077 \\
\hline $\mathrm{C}$ & 9.384771 & -0.176483 & -0.233024 \\
\hline C & 10.642605 & 0.437516 & -0.414368 \\
\hline C & 9.693763 & 2.642293 & -0.294688 \\
\hline $\mathrm{C}$ & 10.788149 & 1.763847 & -0.441854 \\
\hline $\mathrm{C}$ & 9.852085 & 4.060797 & -0.303439 \\
\hline $\mathrm{C}$ & 11.121048 & 4.679839 & -0.324854 \\
\hline $\mathrm{C}$ & 10.137919 & 6.905899 & -0.420287 \\
\hline $\mathrm{C}$ & 11.259098 & 6.060654 & -0.386558 \\
\hline $\mathrm{C}$ & 10.256480 & 8.325593 & -0.511777 \\
\hline $\mathrm{C}$ & 11.482291 & 9.002551 & -0.602852 \\
\hline $\mathrm{H}$ & -12.152264 & -8.337666 & -1.457416 \\
\hline $\mathrm{H}$ & -10.137708 & -9.735442 & -1.391457 \\
\hline $\mathrm{H}$ & -12.019360 & -5.944415 & -1.180010 \\
\hline $\mathrm{H}$ & -11.830487 & -3.907746 & -0.740634 \\
\hline $\mathrm{H}$ & -11.598609 & -1.632187 & -0.526513 \\
\hline $\mathrm{H}$ & -11.324425 & 0.423513 & -0.784771 \\
\hline $\mathrm{H}$ & -11.049273 & 2.683752 & -0.734865 \\
\hline $\mathrm{H}$ & -10.832450 & 4.675038 & -0.275286 \\
\hline $\mathrm{H}$ & -10.544034 & 6.920555 & -0.349198 \\
\hline $\mathrm{H}$ & -10.279572 & 8.947597 & -0.616669 \\
\hline $\mathrm{H}$ & -9.863988 & 11.304507 & -0.754586 \\
\hline $\mathrm{H}$ & -7.583884 & 12.202633 & -0.713183 \\
\hline $\mathrm{H}$ & -7.738528 & -9.950754 & -1.175651 \\
\hline $\mathrm{H}$ & -5.190563 & 11.855592 & -0.601829 \\
\hline $\mathrm{H}$ & -5.358236 & -10.204427 & -1.001616 \\
\hline $\mathrm{H}$ & -2.800525 & 11.536310 & -0.518192 \\
\hline $\mathrm{H}$ & -2.954159 & -10.493521 & -0.876364 \\
\hline $\mathrm{H}$ & -0.404762 & 11.233532 & -0.469425 \\
\hline $\mathrm{H}$ & -0.532180 & -10.780737 & -0.794905 \\
\hline $\mathrm{H}$ & 1.994382 & 10.947432 & -0.459547 \\
\hline $\mathrm{H}$ & 1.884666 & -11.059487 & -0.756562 \\
\hline $\mathrm{H}$ & 4.395625 & 10.677899 & -0.485144 \\
\hline $\mathrm{H}$ & 4.278663 & -11.353365 & -0.774536 \\
\hline $\mathrm{H}$ & 6.797159 & 10.422249 & -0.5346 \\
\hline $\mathrm{H}$ & 6.644101 & -11.673341 & -0.827278 \\
\hline $\mathrm{H}$ & 9.175600 & 10.190585 & -0.594927 \\
\hline $\mathrm{H}$ & 10.209950 & -10.802069 & -0.804044 \\
\hline $\mathrm{H}$ & 8.942328 & -12.108637 & -0.903291 \\
\hline $\mathrm{H}$ & 10.518735 & -8.732077 & -0.723228 \\
\hline $\mathrm{H}$ & 10.831040 & -6.423638 & -0.551602 \\
\hline $\mathrm{H}$ & 11.085329 & -4.389066 & -0.223017 \\
\hline $\mathrm{H}$ & 11.352918 & -2.130546 & -0.143865 \\
\hline $\mathrm{H}$ & 11.543863 & -0.129916 & -0.572226 \\
\hline $\mathrm{H}$ & 11.790230 & 2.116432 & -0.617364 \\
\hline $\mathrm{H}$ & 12.043087 & 4.127308 & -0.271 \\
\hline $\mathrm{H}$ & 12.268054 & 6.438732 & -0.39 \\
\hline $\mathrm{H}$ & 12.442738 & 8.516126 & -0.6 \\
\hline $\mathrm{H}$ & 11.512560 & 10.083969 & -0.668669 \\
\hline 0 & -0.335904 & 1.376222 & 2.626878 \\
\hline $\mathrm{H}$ & 0.138729 & 2.223701 & 2.662504 \\
\hline 0 & -0.738942 & -0.897220 & 2.723995 \\
\hline $\mathrm{C}$ & -0.862818 & -2.132678 & 3.623733 \\
\hline $\mathrm{H}$ & -0.323453 & -0.110285 & 3.173848 \\
\hline $\mathrm{C}$ & -2.236916 & -2.720960 & 3.444950 \\
\hline $\mathrm{C}$ & -2.427489 & -4.094132 & 302 \\
\hline $\mathrm{C}$ & -3.716666 & -4.612172 & 3.183723 \\
\hline C & -4.816487 & -3.761671 & 3.205650 \\
\hline $\mathrm{C}$ & -4.630256 & -2.387843 & 3.367238 \\
\hline $\mathrm{C}$ & -3.347591 & -1.871786 & 3.493657 \\
\hline $\mathrm{H}$ & -1.579722 & -4.770832 & 3.284964 \\
\hline $\mathrm{H}$ & -3.855828 & -5.680722 & 3.058701 \\
\hline $\mathrm{H}$ & -5.817696 & -4.164617 & 3.095151 \\
\hline $\mathrm{H}$ & -5.485248 & -1.720095 & 3.386936 \\
\hline $\mathrm{H}$ & -3.199913 & -0.799175 & 3.595339 \\
\hline C & 0.316901 & -3.0408 & 3.506 \\
\hline $\mathrm{H}$ & 0.2940 & -3.801740 & 2.135 \\
\hline
\end{tabular}




$\begin{array}{lrrr}\mathrm{C} & 1.352625 & -2.945624 & 4.336427 \\ \mathrm{H} & 1.391308 & -2.201304 & 5.128436 \\ \mathrm{H} & 2.199115 & -3.619569 & 4.257833 \\ \mathrm{H} & -0.814317 & -1.610755 & 4.585648\end{array}$

\section{RX2}

C $\quad-11.865554$

$$
-10.774786
$$

$-11.675639$

$-10.377041$

$-11.184807$

$-10.130219$

$-10.956524$

$-9.649917$

$-10.394863$

$-9.375633$

$-10.141380$

$-8.835214$

$-9.591474$

$-8.563822$

$-9.333508$

$-8.016277$

$-8.748653$

$-7.734387$

$-8.433840$

$-7.110450$

$-9.474798$

$-8.385904$

$-9.265702$

$-7.947172$

$-8.797247$

$-7.715786$

$-8.565490$

$-7.186722$

$-8.047457$

$-6.945194$

$-7.766237$

$-6.434847$

$-7.222884$

$-6.161651$

$-6.947969$

$-5.611597$

$-6.383362$

$-5.330363$

$-6.080037$

$-4.750430$

$-7.079071$

$-6.000535$

$-6.855357$

$-5.460186$

$-6.313951$

$-5.232294$

$-6.064716$

$-4.752687$

$-5.559596$

$-4.500802$

$-5.308566$

$-3.978859$

$-4.828722$

$-3.705777$

$-4.552639$

$-3.215782$

$-3.989666$

$-2.931579$

$-3.705572$

$-2.378657$

$-4.680880$

$-3.607388$

$-4.421074$

$-3.090159$

$$
\begin{aligned}
& -7.212228 \\
& -8.073547 \\
& -5.836204 \\
& -5.288447 \\
& -2.881869 \\
& -3.816289 \\
& -1.565937 \\
& -1.042704 \\
& 1.383899 \\
& 0.433704 \\
& \text { 2. } 695752 \\
& \text { 3. } 209811 \\
& 5.579150 \\
& \text { 4. } 610589 \\
& 6.888533 \\
& \text { 7. } 391491 \\
& 9.770497 \\
& 8.787492 \\
& 11.124448 \\
& 11.543267 \\
& -7.563853 \\
& -8.440710 \\
& -6.165162 \\
& -5.658620 \\
& -3.344991 \\
& -4.263787 \\
& -1.945452 \\
& -1.421798 \\
& 0.891539 \\
& -0.093934 \\
& \text { 2. } 284874 \\
& 2.754105 \\
& 5.066836 \\
& 4.141609 \\
& 6.460324 \\
& 6.914366 \\
& \text { 9. } 225285 \\
& 8.292571 \\
& 10.606841 \\
& 11.040299 \\
& -7.954615 \\
& -8.852272 \\
& -6.563910 \\
& -6.060722 \\
& -3.765183 \\
& -4.674460 \\
& -2.383397 \\
& -1.907847 \\
& 0.399477 \\
& -0.565396 \\
& \text { 1. } 789330 \\
& \text { 2. } 249876 \\
& \text { 4. } 611351 \\
& \text { 3. } 651803 \\
& 5.989715 \\
& 6.444096 \\
& 8.742598 \\
& \text { 7. } 817278 \\
& 10.120555 \\
& 10.565254 \\
& -8.397461 \\
& -9.302374 \\
& -6.938710
\end{aligned}
$$




\begin{tabular}{|c|c|c|c|}
\hline $\mathrm{C}$ & -3.912245 & -4.195910 & -0.290607 \\
\hline $\mathrm{C}$ & -2.834362 & -5.112124 & -0.300146 \\
\hline $\mathrm{C}$ & -3.674609 & -2.845132 & -0.142747 \\
\hline $\mathrm{C}$ & -2.299803 & -2.356930 & 0.105722 \\
\hline $\mathrm{C}$ & -3.124885 & -0.077473 & 0.290533 \\
\hline $\mathrm{C}$ & -2.089929 & -1.020635 & 0.394996 \\
\hline $\mathrm{C}$ & -2.877769 & 1.281556 & 0.350189 \\
\hline $\mathrm{C}$ & -1.578382 & 1.773677 & 0.503876 \\
\hline $\mathrm{C}$ & -2.405082 & 4.068383 & 0.089443 \\
\hline $\mathrm{C}$ & -1.296308 & 3.105287 & 0.301622 \\
\hline $\mathrm{C}$ & -2.102324 & 5.467815 & -0.083083 \\
\hline $\mathrm{C}$ & -0.807567 & 5.907342 & -0.101703 \\
\hline $\mathrm{C}$ & -1.594437 & 8.272926 & -0.383896 \\
\hline $\mathrm{C}$ & -0.526277 & 7.358042 & -0.273922 \\
\hline $\mathrm{C}$ & -1.323449 & 9.653126 & -0.523547 \\
\hline $\mathrm{C}$ & 0.000184 & 10.107330 & -0.562526 \\
\hline $\mathrm{C}$ & -2.281975 & -8.852619 & -0.674207 \\
\hline $\mathrm{C}$ & -1.205656 & -9.751363 & -0.746567 \\
\hline $\mathrm{C}$ & -2.014185 & -7.401395 & -0.511455 \\
\hline $\mathrm{C}$ & -0.683358 & -6.940496 & -0.427489 \\
\hline $\mathrm{C}$ & -1.496787 & -4.693227 & -0.184684 \\
\hline C & -0.430602 & -5.570190 & -0.251598 \\
\hline $\mathrm{C}$ & -1.232119 & -3.263208 & 0.061256 \\
\hline $\mathrm{C}$ & 0.076048 & -2.822068 & 0.173032 \\
\hline $\mathrm{C}$ & -0.758253 & -0.637131 & 0.939196 \\
\hline $\mathrm{C}$ & 0.365900 & -1.481610 & 0.436461 \\
\hline $\mathrm{C}$ & -0.461721 & 0.907263 & 1.032469 \\
\hline $\mathrm{C}$ & 0.883680 & 1.303825 & 0.473735 \\
\hline $\mathrm{C}$ & 0.032649 & 3.553043 & 0.252042 \\
\hline $\mathrm{C}$ & 1.101869 & 2.669370 & 0.285656 \\
\hline $\mathrm{C}$ & 0.286554 & 4.984277 & 0.032656 \\
\hline $\mathrm{C}$ & 1.624687 & 5.456689 & -0.103222 \\
\hline $\mathrm{C}$ & 0.806860 & 7.820384 & -0.334375 \\
\hline C & 1.884448 & 6.910888 & -0.268046 \\
\hline $\mathrm{C}$ & 1.064602 & 9.202377 & -0.479317 \\
\hline $\mathrm{C}$ & 2.384124 & 9.665134 & -0.549551 \\
\hline $\mathrm{C}$ & 0.121718 & -9.294786 & -0.683087 \\
\hline $\mathrm{C}$ & 1.195638 & -10.190806 & -0.770553 \\
\hline $\mathrm{C}$ & 0.398184 & -7.841822 & -0.525186 \\
\hline $\mathrm{C}$ & 1.682163 & -7.395295 & -0.485925 \\
\hline $\mathrm{C}$ & 0.903198 & -5.111340 & -0.192248 \\
\hline $\mathrm{C}$ & 1.964992 & -6.024391 & -0.334343 \\
\hline $\mathrm{C}$ & 1.189114 & -3.767771 & -0.038905 \\
\hline $\mathrm{C}$ & 2.505207 & -3.277123 & -0.034926 \\
\hline $\mathrm{C}$ & 1.651846 & -0.979707 & 0.305159 \\
\hline $\mathrm{C}$ & 2.773861 & -1.917244 & 0.060787 \\
\hline $\mathrm{C}$ & 1.907799 & 0.393296 & 0.315741 \\
\hline $\mathrm{C}$ & 3.285865 & 0.887755 & 0.080656 \\
\hline $\mathrm{C}$ & 2.486102 & 3.156926 & 0.065984 \\
\hline $\mathrm{C}$ & 3.514537 & 2.309773 & -0.009781 \\
\hline $\mathrm{C}$ & 2.674290 & 4.580564 & -0.091428 \\
\hline $\mathrm{C}$ & 4.064359 & 5.089694 & -0.237251 \\
\hline $\mathrm{C}$ & 3.210880 & 7.380441 & -0.367221 \\
\hline $\mathrm{C}$ & 4.293938 & 6.473659 & -0.350745 \\
\hline $\mathrm{C}$ & 3.456089 & 8.765898 & -0.500568 \\
\hline $\mathrm{C}$ & 4.770600 & 9.237201 & -0.594988 \\
\hline $\mathrm{C}$ & 2.524582 & -9.734580 & -0.733511 \\
\hline $\mathrm{C}$ & 3.581545 & -10.645636 & -0.828974 \\
\hline $\mathrm{C}$ & 2.805908 & -8.357384 & -0.605903 \\
\hline $\mathrm{C}$ & 4.145725 & -7.895518 & -0.594989 \\
\hline $\mathrm{C}$ & 3.300133 & -5.559102 & -0.333276 \\
\hline $\mathrm{C}$ & 4.429585 & -6.515813 & -0.478786 \\
\hline $\mathrm{C}$ & 3.560691 & -4.184320 & -0.204748 \\
\hline $\mathrm{C}$ & 4.949601 & -3.684656 & -0.258439 \\
\hline $\mathrm{C}$ & 4.077668 & -1.398215 & -0.048923 \\
\hline $\mathrm{C}$ & 5.195546 & -2.356189 & -0.198355 \\
\hline $\mathrm{C}$ & 4.339147 & -0.007400 & -0.050537 \\
\hline C & 5.739714 & 0.483503 & -0.199061 \\
\hline $\mathrm{C}$ & 4.908304 & 2.798161 & -0.183123 \\
\hline $\mathrm{C}$ & 5.984402 & 1.880036 & -0.242743 \\
\hline $\mathrm{C}$ & 5.147762 & 4.183485 & -0.265300 \\
\hline
\end{tabular}




\begin{tabular}{|c|c|c|c|}
\hline C & 6.469056 & 4.664130 & -0.380840 \\
\hline C & 5.614285 & 6.955683 & -0.464521 \\
\hline $\mathrm{C}$ & 6.701698 & 6.052608 & -0.472373 \\
\hline C & 5.847933 & 8.343404 & -0.577856 \\
\hline C & 7.156874 & 8.821674 & -0.682414 \\
\hline C & 4.911598 & -10.201855 & -0.808835 \\
\hline C & 5.951148 & -11.128438 & -0.900356 \\
\hline C & 5.201735 & -8.824515 & -0.700912 \\
\hline C & 6.543953 & -8.382495 & -0.695217 \\
\hline C & 5.772560 & -6.050792 & -0.496956 \\
\hline C & 6.829260 & -6.989834 & -0.609865 \\
\hline C & 6.057108 & -4.659257 & -0.3 \\
\hline C & 7.380283 & -4.171021 & -0.451609 \\
\hline C & 6.580915 & -1.840691 & -0.280686 \\
\hline C & 7.640616 & -2.770093 & -0.393806 \\
\hline C & 6.818564 & -0.436964 & -0.281712 \\
\hline C & 8.142435 & 0.058225 & -0.357819 \\
\hline C & 7.309737 & 2.365156 & -0.345552 \\
\hline C & 8.389408 & 1.458060 & -0.377250 \\
\hline C & 7.554250 & 3.758515 & -0.406453 \\
\hline C & 8.879265 & 4.248143 & -0.509763 \\
\hline C & 8.018957 & 6.543683 & -0.584214 \\
\hline C & 9.112359 & 5.650339 & -0.601741 \\
\hline C & 8.239541 & 7.933644 & -0.684927 \\
\hline C & 9.539035 & 8.428603 & -0.791400 \\
\hline C & 7.293007 & -10.722951 & -0.884996 \\
\hline C & 8.263954 & -11.730384 & -0.991436 \\
\hline C & 7.600877 & -9.333786 & -0.775022 \\
\hline C & 8.924954 & -8.860178 & -0.727861 \\
\hline C & 8.171543 & -6.545137 & -0.626568 \\
\hline C & 9.201832 & -7.501336 & -0.649998 \\
\hline C & 8.455070 & -5.074620 & -0.597173 \\
\hline C & 9.755116 & -4.556615 & -0.758716 \\
\hline C & 8.969678 & -2.307972 & -0.483322 \\
\hline C & 9.999000 & -3.243605 & -0.706275 \\
\hline C & 9.233187 & -0.838278 & -0.402393 \\
\hline C & 10.538213 & -0.319701 & -0.340225 \\
\hline C & 9.724879 & 1.939953 & -0.427360 \\
\hline C & 10.772629 & 0.994422 & -0.352620 \\
\hline C & 9.970097 & 3.342149 & -0.528088 \\
\hline C & 11.269997 & 3.872130 & -0.679110 \\
\hline C & 10.435278 & 6.159710 & -0.721138 \\
\hline C & 11.495643 & 5.239889 & -0.766813 \\
\hline C & 10.645641 & 7.569196 & -0.807010 \\
\hline C & 11.912042 & 8.165511 & -0 . \\
\hline $\mathrm{H}$ & -12.868100 & -7.613118 & -1.032502 \\
\hline $\mathrm{H}$ & -10.947981 & -9.139908 & -1.035715 \\
\hline $\mathrm{H}$ & -12.563377 & -5.227102 & -0.857671 \\
\hline $\mathrm{H}$ & -12.217374 & -3.188581 & -0.555528 \\
\hline $\mathrm{H}$ & -11.823421 & -0.927827 & -0.430646 \\
\hline $\mathrm{H}$ & -11.417249 & 1.091092 & -0.817352 \\
\hline $\mathrm{H}$ & -10.992348 & 3.329583 & -0.819250 \\
\hline $\mathrm{H}$ & -10.634458 & 5.314822 & -0.443144 \\
\hline $\mathrm{H}$ & -10.195747 & 7.532709 & -0.551475 \\
\hline $\mathrm{H}$ & -9.797189 & 9.533498 & -0.841552 \\
\hline $\mathrm{H}$ & -9.225914 & 11.856181 & -1.000412 \\
\hline $\mathrm{H}$ & -6.892034 & 12.601729 & -0.958525 \\
\hline $\mathrm{H}$ & -8.557789 & -9.508462 & -0.953699 \\
\hline $\mathrm{H}$ & -4.528105 & 12.098558 & -0.834196 \\
\hline $\mathrm{H}$ & -6.191150 & -9.915894 & -0.896291 \\
\hline $\mathrm{H}$ & -2.166257 & 11.624319 & -0.735603 \\
\hline $\mathrm{H}$ & -3.805269 & -10.362932 & -0.869361 \\
\hline $\mathrm{H}$ & 0.202626 & 11.167507 & -0.673607 \\
\hline $\mathrm{H}$ & -1.401879 & -10.811588 & -0.863645 \\
\hline $\mathrm{H}$ & 2.577076 & 10.727313 & -0.658394 \\
\hline $\mathrm{H}$ & 0.996266 & -11.251748 & -0.877534 \\
\hline $\mathrm{H}$ & 4.955216 & 10.301971 & -0.69 \\
\hline $\mathrm{H}$ & 3.368393 & -11.705632 & -0.921106 \\
\hline $\mathrm{H}$ & 7.333258 & 9.889107 & -0.767087 \\
\hline $\mathrm{H}$ & 5.708966 & -12.183494 & -0.985726 \\
\hline $\mathrm{H}$ & 9.688864 & 9.501792 & -0.862680 \\
\hline
\end{tabular}




\begin{tabular}{lrrr} 
& & & \\
$\mathrm{H}$ & 9.325138 & -11.548601 & -1.013940 \\
$\mathrm{H}$ & 7.973610 & -12.771347 & -1.071828 \\
$\mathrm{H}$ & 9.777117 & -9.519444 & -0.742948 \\
$\mathrm{H}$ & 10.242264 & -7.227015 & -0.594271 \\
$\mathrm{H}$ & 10.600857 & -5.195255 & -0.954877 \\
$\mathrm{H}$ & 11.019386 & -2.936045 & -0.868695 \\
$\mathrm{H}$ & 11.399982 & -0.963401 & -0.264617 \\
$\mathrm{H}$ & 11.807628 & 1.282893 & -0.284612 \\
$\mathrm{H}$ & 12.147784 & 3.255168 & -0.763044 \\
$\mathrm{H}$ & 12.521395 & 5.546959 & -0.887308 \\
$\mathrm{H}$ & 12.839901 & 7.619229 & -0.906480 \\
$\mathrm{H}$ & 12.011546 & 9.242775 & -0.970386 \\
$\mathrm{O}$ & -0.396802 & 1.261801 & 2.449532 \\
$\mathrm{O}$ & -0.808777 & -1.019984 & 2.453415 \\
$\mathrm{C}$ & -1.351493 & -2.200270 & 3.179410 \\
$\mathrm{H}$ & 0.414441 & 1.785135 & 2.592455 \\
$\mathrm{C}$ & -0.783157 & -2.100439 & 4.564110 \\
$\mathrm{C}$ & -2.853787 & -2.214169 & 3.173785 \\
$\mathrm{H}$ & -0.934914 & -3.054647 & 2.641612 \\
$\mathrm{C}$ & -3.495189 & -3.445904 & 3.043887 \\
$\mathrm{C}$ & -4.885575 & -3.512494 & 3.064448 \\
$\mathrm{C}$ & -5.634901 & -2.347664 & 3.215013 \\
$\mathrm{C}$ & -4.992680 & -1.119529 & 3.361233 \\
$\mathrm{C}$ & -3.602648 & -1.051362 & 3.350418 \\
$\mathrm{H}$ & -2.906887 & -4.347969 & 2.891176 \\
$\mathrm{H}$ & -5.380749 & -4.469252 & 2.936980 \\
$\mathrm{H}$ & -6.718687 & -2.394768 & 3.208035 \\
$\mathrm{H}$ & -5.574942 & -0.209918 & 3.468253 \\
$\mathrm{H}$ & -3.102884 & -0.087459 & 3.424175 \\
$\mathrm{C}$ & 0.171108 & -2.919451 & 5.000678 \\
$\mathrm{H}$ & -1.235798 & -1.355023 & 5.216595 \\
$\mathrm{H}$ & 0.544752 & -2.866376 & 6.017366 \\
$\mathrm{H}$ & 0.595324 & -3.686077 & 4.355963 \\
$\mathrm{C}$ & 2.646354 & -0.305200 & 3.563645 \\
$\mathrm{C}$ & 3.894009 & -0.726405 & 3.223244 \\
$\mathrm{C}$ & 4.829379 & 0.353587 & 3.109134 \\
$\mathrm{C}$ & 4.279989 & 1.574958 & 3.360394 \\
$\mathrm{~S}$ & 2.607294 & 1.408331 & 3.793631 \\
$\mathrm{H}$ & 1.760521 & -0.913383 & 3.697202 \\
$\mathrm{H}$ & 4.145290 & -1.765860 & 3.044900 \\
$\mathrm{H}$ & 5.872872 & 0.224197 & 2.844937 \\
$\mathrm{H}$ & -0.586163 & -0.164327 & 2.939002 \\
$\mathrm{C}$ & 4.943759 & 2.925981 & 3.350876 \\
$\mathrm{H}$ & 4.338039 & 3.640241 & 2.785272 \\
$\mathrm{H}$ & 5.079141 & 3.300617 & 4.369264 \\
$\mathrm{H}$ & 5.919476 & 2.827103 & 2.870171 \\
& & & \\
\hline
\end{tabular}

\section{TS2 - $\alpha$ attack}

\begin{tabular}{|c|c|c|c|}
\hline $\mathrm{C}$ & -10.312108 & -9.234875 & -0.992999 \\
\hline $\mathrm{C}$ & -9.076116 & -9.870892 & -1.023999 \\
\hline $\mathrm{C}$ & -10.388089 & -7.849874 & -0.860999 \\
\hline C & -9.218078 & -7.063890 & -0.750999 \\
\hline $\mathrm{C}$ & -10.468048 & -4.850873 & -0.576999 \\
\hline $\mathrm{C}$ & -9.256058 & -5.570890 & -0.619999 \\
\hline $\mathrm{C}$ & -10.492030 & -3.514873 & -0.498999 \\
\hline $\mathrm{C}$ & -9.307019 & -2.753889 & -0.459999 \\
\hline $\mathrm{C}$ & -10.494989 & -0.509873 & -0.648999 \\
\hline $\mathrm{C}$ & -9.316999 & -1.250889 & -0.44399 \\
\hline C & -10.493970 & 0.826127 & -0.63999 \\
\hline C & -9.31 & 1.568111 & -0 \\
\hline C & -10.506 & 3.814128 & -0 \\
\hline C & -9.31 & 3.065111 & -0 . \\
\hline C & -10.500911 & 5.148127 & -0.4 \\
\hline $\mathrm{C}$ & -9.298901 & 5.889111 & -0.520999 \\
\hline C & -10.460871 & 8.083127 & -0.814999 \\
\hline $\mathrm{C}$ & -9.282882 & 7.312111 & -0.642999 \\
\hline $\mathrm{C}$ & -10.405852 & 9.471126 & -0.927999 \\
\hline $\mathrm{C}$ & -9.18 & 10.134110 & -0.8819 \\
\hline C & 06 & -9.12 & -0 . \\
\hline$c$ & -6.6 & -9 . & -0 \\
\hline
\end{tabular}




\begin{tabular}{|c|c|c|c|}
\hline $\mathrm{C}$ & -7.960087 & -7.712907 & -0.781999 \\
\hline $\mathrm{C}$ & -6.763077 & -6.966924 & -0.673999 \\
\hline $\mathrm{C}$ & -8.037048 & -4.856906 & -0.526999 \\
\hline C & -6.803058 & -5.556923 & -0.535999 \\
\hline $\mathrm{C}$ & -8.073029 & -3.436906 & -0.429999 \\
\hline $\mathrm{C}$ & -6.819018 & -2.662923 & -0.279999 \\
\hline C & -8.100989 & -0.547905 & -0.282999 \\
\hline $\mathrm{C}$ & -6.831999 & -1.307923 & -0.184999 \\
\hline $\mathrm{C}$ & -8.090970 & 0.877095 & -0.269999 \\
\hline $\mathrm{C}$ & -6.877960 & 1.605078 & -0.141999 \\
\hline $\mathrm{C}$ & -8.083930 & 3.753094 & -0.339999 \\
\hline $\mathrm{C}$ & -6.872940 & 3.033078 & -0.197999 \\
\hline $\mathrm{C}$ & -8.074911 & 5.175094 & -0.407999 \\
\hline C & -6.847901 & 5.873078 & -0.364999 \\
\hline $\mathrm{C}$ & -8.038872 & 7.997094 & -0.610999 \\
\hline $\mathrm{C}$ & -6.829882 & 7.281077 & -0.466999 \\
\hline $\mathrm{C}$ & -7.999853 & 9.411093 & -0.728999 \\
\hline $\mathrm{C}$ & -6.776844 & 10.089077 & -0.700999 \\
\hline C & -5.469105 & -9.049941 & -0.868999 \\
\hline $\mathrm{C}$ & -4.236114 & -9.719958 & -0.935999 \\
\hline $\mathrm{C}$ & -5.517086 & -7.645941 & -0.713999 \\
\hline $\mathrm{C}$ & -4.244076 & -6.887958 & -0.596999 \\
\hline $\mathrm{C}$ & -5.524047 & -4.806941 & -0.396999 \\
\hline $\mathrm{C}$ & -4.287057 & -5.491957 & -0.422999 \\
\hline $\mathrm{C}$ & -5.541028 & -3.408940 & -0.244999 \\
\hline $\mathrm{C}$ & -4.343019 & -2.710957 & -0.072999 \\
\hline $\mathrm{C}$ & -5.570989 & -0.548940 & -0.004999 \\
\hline $\mathrm{C}$ & -4.360999 & -1.250956 & 0.097001 \\
\hline $\mathrm{C}$ & -5.590970 & 0.861060 & 0.027001 \\
\hline $\mathrm{C}$ & -4.389960 & 1.574044 & 0.177001 \\
\hline C & -5.655930 & 3.757061 & -0.114999 \\
\hline $\mathrm{C}$ & -4.381940 & 3.039044 & 0.079001 \\
\hline $\mathrm{C}$ & -5.635911 & 5.165061 & -0.215999 \\
\hline $\mathrm{C}$ & -4.404901 & 5.867044 & -0.176999 \\
\hline $\mathrm{C}$ & -5.597873 & 7.977060 & -0.439999 \\
\hline $\mathrm{C}$ & -4.384882 & 7.269044 & -0.300999 \\
\hline $\mathrm{C}$ & -5.577853 & 9.384060 & -0.560999 \\
\hline $\mathrm{C}$ & -4.358844 & 10.073044 & -0.552999 \\
\hline $\mathrm{C}$ & -3.028105 & -9.017975 & -0.849999 \\
\hline $\mathrm{C}$ & -1.798114 & -9.690991 & -0.944999 \\
\hline $\mathrm{C}$ & -3.053085 & -7.542974 & -0.661999 \\
\hline $\mathrm{C}$ & -1.834075 & -6.839991 & -0.560999 \\
\hline $\mathrm{C}$ & -3.082047 & -4.772974 & -0.281999 \\
\hline $\mathrm{C}$ & -1.847056 & -5.449991 & -0.356999 \\
\hline C & -3.121028 & -3.385973 & -0.081999 \\
\hline $\mathrm{C}$ & -1.864018 & -2.637991 & 0.143001 \\
\hline $\mathrm{C}$ & -3.088989 & -0.537974 & 0.352001 \\
\hline $\mathrm{C}$ & -1.914999 & -1.266990 & 0.450001 \\
\hline C & -3.106970 & 0.862026 & 0.391001 \\
\hline $\mathrm{C}$ & -1.939960 & 1.591011 & 0.515001 \\
\hline $\mathrm{C}$ & -3.161931 & 3.730027 & 0.131001 \\
\hline $\mathrm{C}$ & -1.900941 & 2.980010 & 0.335001 \\
\hline $\mathrm{C}$ & -3.119912 & 5.128027 & -0.015999 \\
\hline $\mathrm{C}$ & -1.932902 & 5.799010 & -0.028999 \\
\hline $\mathrm{C}$ & -3.155873 & 7.970027 & -0.301999 \\
\hline $\mathrm{C}$ & -1.932882 & 7.274010 & -0.189999 \\
\hline $\mathrm{C}$ & -3.149854 & 9.377027 & -0.432999 \\
\hline C & -1.934844 & 10.073010 & -0.455999 \\
\hline $\mathrm{C}$ & -0.586104 & -8.990008 & -0.871999 \\
\hline $\mathrm{C}$ & 0.641886 & -9.656025 & -0.987999 \\
\hline $\mathrm{C}$ & -0.602084 & -7.518008 & -0.670999 \\
\hline $\mathrm{C}$ & 0.613925 & -6.801024 & -0.608999 \\
\hline $\mathrm{C}$ & -0.633046 & -4.744007 & -0.257999 \\
\hline $\mathrm{C}$ & 0.597945 & -5.408024 & -0.409999 \\
\hline $\mathrm{C}$ & -0.652027 & -3.293007 & 0.012001 \\
\hline $\mathrm{C}$ & 0.559983 & -2.582024 & 0.045001 \\
\hline C & -0.645990 & -0.632007 & 0.936001 \\
\hline $\mathrm{C}$ & 0.584002 & -1.226024 & 0.308001 \\
\hline $\mathrm{C}$ & -0.671969 & 0.942993 & 1.028001 \\
\hline $\mathrm{C}$ & 0.577040 & 008 & 0.49 \\
\hline $\mathrm{C}$ & & & 0.25 \\
\hline
\end{tabular}




\begin{tabular}{|c|c|c|c|}
\hline $\mathrm{C}$ & 0.538059 & 2.976977 & 0.326001 \\
\hline $\mathrm{C}$ & -0.695912 & 5.118994 & 0.084001 \\
\hline $\mathrm{C}$ & 0.538098 & 5.802977 & -0.019999 \\
\hline C & -0.709873 & 7.977994 & -0.236999 \\
\hline C & 0.519118 & 7.284977 & -0.170999 \\
\hline C & -0.717853 & 9.384994 & -0.368999 \\
\hline C & 0.491156 & 10.087977 & -0.425999 \\
\hline C & 1.853896 & -8.949041 & -0.933999 \\
\hline $\mathrm{C}$ & 3.076887 & -9.618058 & -1.054999 \\
\hline C & 1.845916 & -7.472041 & -0.746999 \\
\hline C & 3.018926 & -6.785057 & -0.714999 \\
\hline $\mathrm{C}$ & 1.816954 & -4.693041 & -0.382999 \\
\hline $\mathrm{C}$ & 3.033945 & -5.386057 & -0.544999 \\
\hline $\mathrm{C}$ & 1.830973 & -3.298041 & -0.200999 \\
\hline $\mathrm{C}$ & 3.043983 & -2.588058 & -0.207999 \\
\hline C & 1.771012 & -0.479040 & 0.218001 \\
\hline $\mathrm{C}$ & 3.044002 & -1.190058 & -0.039999 \\
\hline $\mathrm{C}$ & 1.769031 & 0.896960 & 0.314001 \\
\hline C & 3.028041 & 1.656943 & 0.111001 \\
\hline $\mathrm{C}$ & 1.802069 & 3.729959 & 0.116001 \\
\hline C & 2.990060 & 3.065943 & 0.050001 \\
\hline $\mathrm{C}$ & 1.734089 & 5.138960 & -0.006999 \\
\hline $\mathrm{C}$ & 3.003099 & 5.904943 & -0.144999 \\
\hline $\mathrm{C}$ & 1.734128 & 7.995960 & -0.258999 \\
\hline $\mathrm{C}$ & 2.968118 & 7.309943 & -0.246999 \\
\hline $\mathrm{C}$ & 1.713147 & 9.404961 & -0.379999 \\
\hline $\mathrm{C}$ & 2.915157 & 10.115944 & -0.465999 \\
\hline C & 4.291897 & -8.916075 & -1.014999 \\
\hline $\mathrm{C}$ & 5.500887 & -9.608091 & -1.130999 \\
\hline $\mathrm{C}$ & 4.304916 & -7.513075 & -0.857999 \\
\hline $\mathrm{C}$ & 5.531925 & -6.807092 & -0.831999 \\
\hline C & 4.256955 & -4.676074 & -0.534999 \\
\hline C & 5.548945 & -5.402092 & -0.683999 \\
\hline $\mathrm{C}$ & 4.252974 & -3.280074 & -0.379999 \\
\hline $\mathrm{C}$ & 5.525984 & -2.532091 & -0.386999 \\
\hline $\mathrm{C}$ & 4.244012 & -0.458074 & -0.104999 \\
\hline $\mathrm{C}$ & 5.521002 & -1.185091 & -0.273999 \\
\hline $\mathrm{C}$ & 4.242031 & 0.951926 & -0.042999 \\
\hline $\mathrm{C}$ & 5.523042 & 1.701909 & -0.173999 \\
\hline $\mathrm{C}$ & 4.267070 & 3.814926 & -0.112999 \\
\hline $\mathrm{C}$ & 5.498061 & 3.118909 & -0.190999 \\
\hline $\mathrm{C}$ & 4.239090 & 5.220926 & -0.181999 \\
\hline C & 5.446100 & 5.944910 & -0.296999 \\
\hline C & 4.174128 & 8.033927 & -0.356999 \\
\hline $\mathrm{C}$ & 5.412119 & 7.352910 & -0.375999 \\
\hline $\mathrm{C}$ & 4.142147 & 9.441927 & -0.457999 \\
\hline $\mathrm{C}$ & 5.337157 & 10.159911 & -0.559999 \\
\hline $\mathrm{C}$ & 6.721897 & -8.922108 & -1.093999 \\
\hline $\mathrm{C}$ & 7.916887 & -9.634124 & -1.199999 \\
\hline $\mathrm{C}$ & 6.744916 & -7.518108 & -0.950999 \\
\hline $\mathrm{C}$ & 7.979925 & -6.832125 & -0.919999 \\
\hline $\mathrm{C}$ & 6.780954 & -4.694109 & -0.672999 \\
\hline $\mathrm{C}$ & 7.996944 & -5.414125 & -0.794999 \\
\hline $\mathrm{C}$ & 6.797974 & -3.278109 & -0.534999 \\
\hline $\mathrm{C}$ & 8.007984 & -2.550125 & -0.554999 \\
\hline $\mathrm{C}$ & 6.786013 & -0.418109 & -0.322999 \\
\hline $\mathrm{C}$ & 8.001003 & -1.128125 & -0.454999 \\
\hline $\mathrm{C}$ & 6.756032 & 1.003892 & -0.281999 \\
\hline $\mathrm{C}$ & 7.962042 & 1.740875 & -0.347999 \\
\hline $\mathrm{C}$ & 6.707071 & 3.846892 & -0.293999 \\
\hline $\mathrm{C}$ & 7.939062 & 3.160876 & 42999 \\
\hline $\mathrm{C}$ & 6.683090 & 5.261893 & -0.337999 \\
\hline $\mathrm{C}$ & 7.892100 & 5.993876 & -0.439999 \\
\hline $\mathrm{C}$ & 6.613129 & 8.084894 & 999 \\
\hline $\mathrm{C}$ & 7.856120 & 7.414877 & 6999 \\
\hline $\mathrm{C}$ & 6.567148 & 9.491894 & -0.573999 \\
\hline $\mathrm{C}$ & 7.750158 & 10.224878 & -0.679999 \\
\hline $\mathrm{C}$ & 9.157896 & -8.984141 & -1.161999 \\
\hline $\mathrm{C}$ & 10.300885 & -9.788157 & -1.284999 \\
\hline $\mathrm{C}$ & 9.196 & -7.566142 & -1.011999 \\
\hline $\mathrm{C}$ & 10.407925 & -6.854158 & -0.933999 \\
\hline
\end{tabular}




\begin{tabular}{|c|c|c|c|}
\hline $\mathrm{C}$ & 9.231954 & -4.726142 & -0.781999 \\
\hline C & 10.423944 & -5.471158 & -0.815999 \\
\hline C & 9.233974 & -3.232142 & -0.709999 \\
\hline C & 10.414985 & -2.476158 & -0.840999 \\
\hline C & 9.220013 & -0.423142 & -0.520999 \\
\hline C & 10.408003 & -1.143158 & -0.753999 \\
\hline C & 9.202033 & 1.066858 & -0.409999 \\
\hline C & 10.385043 & 1.821842 & -0.341999 \\
\hline $\mathrm{C}$ & 9.159071 & 3.886859 & -0.390999 \\
\hline $\mathrm{C}$ & 10.367061 & 3.155842 & -0.332999 \\
\hline C & 9.134091 & 5.310859 & -0.473999 \\
\hline C & 10.310101 & 6.076843 & -0.621999 \\
\hline $\mathrm{C}$ & 9.058130 & 8.165860 & -0.635999 \\
\hline $\mathrm{C}$ & 10.273120 & 7.462844 & -0.694999 \\
\hline $\mathrm{C}$ & 8.999149 & 9.589861 & -0.707999 \\
\hline $\mathrm{C}$ & 10.130161 & 10.413846 & -0.804999 \\
\hline $\mathrm{H}$ & -11.219116 & -9.819863 & -1.074999 \\
\hline $\mathrm{H}$ & -9.042131 & -10.948892 & -1.130999 \\
\hline $\mathrm{H}$ & -11.377083 & -7.422861 & -0.852999 \\
\hline $\mathrm{H}$ & -11.424055 & -5.345860 & -0.604999 \\
\hline $\mathrm{H}$ & -11.464023 & -3.050859 & -0.455999 \\
\hline $\mathrm{H}$ & -11.438995 & -0.990860 & -0.848999 \\
\hline $\mathrm{H}$ & -11.438964 & 1.304140 & -0.835999 \\
\hline $\mathrm{H}$ & -11.474936 & 3.341141 & -0.396999 \\
\hline $\mathrm{H}$ & -11.468905 & 5.619141 & -0.505999 \\
\hline $\mathrm{H}$ & -11.443877 & 7.650140 & -0.882999 \\
\hline $\mathrm{H}$ & -11.319844 & 10.038139 & -1.058999 \\
\hline $\mathrm{H}$ & -9.170828 & 11.214109 & -0.975999 \\
\hline $\mathrm{H}$ & -6.624130 & -10.853926 & -1.081999 \\
\hline $\mathrm{H}$ & -6.757829 & 11.169076 & -0.794999 \\
\hline $\mathrm{H}$ & -4.218129 & -10.796958 & -1.064999 \\
\hline $\mathrm{H}$ & -4.349829 & 11.153043 & -0.651999 \\
\hline $\mathrm{H}$ & -1.786129 & -10.765992 & -1.087999 \\
\hline $\mathrm{H}$ & -1.934829 & 11.154010 & -0.558999 \\
\hline $\mathrm{H}$ & 0.654872 & -10.731025 & -1.129999 \\
\hline $\mathrm{H}$ & 0.482171 & 11.167977 & -0.524999 \\
\hline $\mathrm{H}$ & 3.083872 & -10.695058 & -1.183999 \\
\hline $\mathrm{H}$ & 2.896171 & 11.197944 & -0.553999 \\
\hline $\mathrm{H}$ & 5.491872 & -10.687091 & -1.247999 \\
\hline $\mathrm{H}$ & 5.308172 & 11.241912 & -0.633999 \\
\hline $\mathrm{H}$ & 7.877872 & -10.713124 & -1.313999 \\
\hline $\mathrm{H}$ & 7.695173 & 11.306879 & -0.740999 \\
\hline $\mathrm{H}$ & 11.308890 & -9.409170 & -1.294999 \\
\hline $\mathrm{H}$ & 10.211870 & -10.862155 & -1.394999 \\
\hline $\mathrm{H}$ & 11.368918 & -7.341171 & -0.953999 \\
\hline $\mathrm{H}$ & 11.392950 & -5.009172 & -0.736999 \\
\hline $\mathrm{H}$ & 11.366978 & -2.939171 & -1.041999 \\
\hline $\mathrm{H}$ & 11.355010 & -0.647171 & -0.895999 \\
\hline $\mathrm{H}$ & 11.354037 & 1.352829 & -0.279999 \\
\hline $\mathrm{H}$ & 11.330068 & 3.632829 & -0.262999 \\
\hline $\mathrm{H}$ & 11.288095 & 5.637830 & -0.715999 \\
\hline $\mathrm{H}$ & 11.222127 & 7.958831 & -0.814999 \\
\hline $\mathrm{H}$ & 11.144156 & 10.052832 & -0.816999 \\
\hline $\mathrm{H}$ & 10.025175 & 11.491847 & -0.860999 \\
\hline 0 & -0.687965 & 1.250993 & 2.455001 \\
\hline O & -0.432996 & -1.085010 & 2.380001 \\
\hline C & -0.997014 & -2.340002 & 3.276001 \\
\hline $\mathrm{H}$ & -0.375952 & 2.166989 & 2.553001 \\
\hline C & -0.163012 & -2.228014 & 4.465001 \\
\hline $\mathrm{C}$ & -2.481012 & -2.203982 & 3.383001 \\
\hline $\mathrm{H}$ & -0.703025 & -3.165006 & 2.627001 \\
\hline C & -3.260027 & -3.342971 & 3.171001 \\
\hline $\mathrm{C}$ & -4.648026 & -3.265952 & 3.236001 \\
\hline C & -5.262010 & -2.043944 & 3.498001 \\
\hline C & -4.485994 & -0.906955 & 3.720001 \\
\hline C & -3.097995 & -0.986974 & 3.674001 \\
\hline $\mathrm{H}$ & -2.777040 & -4.287978 & 2.931001 \\
\hline $\mathrm{H}$ & -5.245038 & -4.152944 & 3.052001 \\
\hline $\mathrm{H}$ & -6.344009 & -1.974929 & 3.522001 \\
\hline $\mathrm{H}$ & -4.962981 & 0.047052 & 3.916001 \\
\hline $\mathrm{H}$ & -2.505983 & -0.083982 & 3.805001 \\
\hline
\end{tabular}




$\begin{array}{rrrr}\mathrm{C} & 1.028979 & -2.889030 & 4.513001 \\ \mathrm{H} & -0.445002 & -1.503010 & 5.224001 \\ \mathrm{H} & 1.641979 & -2.862038 & 5.408001 \\ \mathrm{H} & 1.166967 & -3.764032 & 3.884001 \\ \mathrm{C} & 2.510991 & -2.013050 & 3.264001 \\ \mathrm{C} & 4.842992 & -1.929082 & 4.183001 \\ \mathrm{H} & 1.774989 & -2.146040 & 2.484001 \\ \mathrm{H} & -0.405985 & -0.250010 & 2.921001 \\ \mathrm{C} & 6.228987 & -2.265101 & 4.634001 \\ \mathrm{H} & 6.236975 & -3.148101 & 5.280001 \\ \mathrm{H} & 6.881985 & -2.470110 & 3.779001 \\ \mathrm{H} & 6.648999 & -1.426107 & 5.193001 \\ \mathrm{C} & 2.830007 & -0.808055 & 3.856001 \\ \mathrm{H} & 2.124019 & 0.014955 & 3.915001 \\ \mathrm{~S} & 3.907977 & -3.057069 & 3.265001 \\ \mathrm{C} & 4.130008 & -0.779072 & 4.415001 \\ \mathrm{H} & 4.534019 & 0.051922 & 4.981001\end{array}$

\section{TS2 - $\boldsymbol{\beta}$ attack}

\begin{tabular}{|c|c|c|c|}
\hline $\mathrm{C}$ & -10.062810 & -9.446211 & -1.205017 \\
\hline $\mathrm{C}$ & -8.812798 & -10.056186 & -1.210017 \\
\hline $\mathrm{C}$ & -10.170837 & -8.064213 & -1.050017 \\
\hline $\mathrm{C}$ & -9.019853 & -7.258190 & -0.886017 \\
\hline $\mathrm{C}$ & -10.320897 & -5.080216 & -0.653017 \\
\hline $\mathrm{C}$ & -9.092883 & -5.770192 & -0.720017 \\
\hline $\mathrm{C}$ & -10.376924 & -3.745218 & -0.554017 \\
\hline $\mathrm{C}$ & -9.209939 & -2.957194 & -0.517017 \\
\hline $\mathrm{C}$ & -10.449984 & -0.737219 & -0.679017 \\
\hline $\mathrm{C}$ & -9.253969 & -1.454195 & -0.485017 \\
\hline $\mathrm{C}$ & -10.479010 & 0.599780 & -0.650017 \\
\hline $\mathrm{C}$ & -9.316026 & 1.365804 & -0.421017 \\
\hline $\mathrm{C}$ & -10.558070 & 3.583779 & -0.351017 \\
\hline $\mathrm{C}$ & -9.349056 & 2.861803 & -0.373017 \\
\hline $\mathrm{C}$ & -10.583097 & 4.920778 & -0.384017 \\
\hline C & -9.398112 & 5.688802 & -0.435017 \\
\hline $\mathrm{C}$ & -10.612155 & 7.864778 & -0.651017 \\
\hline $\mathrm{C}$ & -9.413140 & 7.114802 & -0.530017 \\
\hline $\mathrm{C}$ & -10.588183 & 9.255778 & -0.740017 \\
\hline $\mathrm{C}$ & -9.380197 & 9.941802 & -0.721017 \\
\hline $\mathrm{C}$ & -7.652813 & -9.287163 & -1.064017 \\
\hline $\mathrm{C}$ & -6.401800 & -9.913138 & -1.088017 \\
\hline $\mathrm{C}$ & -7.747841 & -7.881165 & -0.902017 \\
\hline $\mathrm{C}$ & -6.568856 & -7.112142 & -0.762017 \\
\hline $\mathrm{C}$ & -7.890898 & -5.030168 & -0.610017 \\
\hline $\mathrm{C}$ & -6.641885 & -5.703143 & -0.613017 \\
\hline $\mathrm{C}$ & -7.959926 & -3.612169 & -0.497017 \\
\hline $\mathrm{C}$ & -6.723942 & -2.812145 & -0.341017 \\
\hline $\mathrm{C}$ & -8.053984 & -0.726171 & -0.323017 \\
\hline $\mathrm{C}$ & -6.767969 & -1.458146 & -0.237017 \\
\hline C & -8.076012 & 0.698828 & -0.293017 \\
\hline $\mathrm{C}$ & -6.879027 & 1.452852 & -0.173017 \\
\hline $\mathrm{C}$ & -8.133070 & 3.576827 & -0.325017 \\
\hline $\mathrm{C}$ & -6.905056 & 2.881852 & -0.217017 \\
\hline $\mathrm{C}$ & -8.156098 & 4.998827 & -0.369017 \\
\hline $\mathrm{C}$ & -6.943113 & 5.723851 & -0.348017 \\
\hline $\mathrm{C}$ & -8.182155 & 7.824826 & -0.524017 \\
\hline $\mathrm{C}$ & -6.956141 & 7.131851 & -0.428017 \\
\hline $\mathrm{C}$ & -8.175183 & 9.240826 & -0.619017 \\
\hline $\mathrm{C}$ & -6.965197 & 9.943851 & -0.617017 \\
\hline $\mathrm{C}$ & -5.229815 & -9.166115 & -0.953017 \\
\hline $\mathrm{C}$ & -3.982803 & -9.811090 & -0.998017 \\
\hline $\mathrm{C}$ & -5.309843 & -7.765116 & -0.783017 \\
\hline $\mathrm{C}$ & -4.054859 & -6.983091 & -0.633017 \\
\hline $\mathrm{C}$ & -5.380900 & -4.929118 & -0.451017 \\
\hline $\mathrm{C}$ & -4.129887 & -5.589093 & -0.455017 \\
\hline $\mathrm{C}$ & -5.429928 & -3.531119 & -0.300017 \\
\hline $\mathrm{C}$ & -4.248942 & -2.808095 & -0.115017 \\
\hline $\mathrm{C}$ & -5.524985 & -0.672121 & -0.059017 \\
\hline $\mathrm{C}$ & -4.299972 & -1.347096 & 0.042983 \\
\hline C & & 0 . & 25017 \\
\hline
\end{tabular}




\begin{tabular}{|c|c|c|c|}
\hline C & -4.389028 & 1.473902 & 0.113983 \\
\hline C & -5.703071 & 3.630876 & -0.150017 \\
\hline C & -4.412057 & 2.938902 & 0.015983 \\
\hline C & -5.714099 & 5.038876 & -0.238017 \\
\hline C & -4.496114 & 5.766900 & -0.218017 \\
\hline C & -5.737155 & 7.853875 & -0.424017 \\
\hline C & -4.506142 & 7.169900 & -0.325017 \\
\hline C & -5.748183 & 9.262875 & -0.524017 \\
\hline $\mathrm{C}$ & -4.543198 & 9.975899 & -0.538017 \\
\hline C & -2.791817 & -9.088066 & -0.875017 \\
\hline C & -1.547804 & -9.737041 & -0.949017 \\
\hline C & -2.850846 & -7.615067 & -0.676017 \\
\hline C & -1.647861 & -6.889043 & -0.545017 \\
\hline $\mathrm{C}$ & -2.941902 & -4.847069 & -0.289017 \\
\hline $\mathrm{C}$ & -1.692889 & -5.500044 & -0.337017 \\
\hline $\mathrm{C}$ & -3.010929 & -3.459071 & -0.098017 \\
\hline $\mathrm{C}$ & -1.772945 & -2.682046 & 0.136983 \\
\hline $\mathrm{C}$ & -3.044986 & -0.605071 & 0.293983 \\
\hline C & -1.854972 & -1.310047 & 0.412983 \\
\hline C & -3.091014 & 0.790928 & 0.322983 \\
\hline $\mathrm{C}$ & -1.939029 & 1.547951 & 0.442983 \\
\hline C & -3.207071 & 3.654926 & 0.049983 \\
\hline $\mathrm{C}$ & -1.931057 & 2.930951 & 0.243983 \\
\hline $\mathrm{C}$ & -3.193099 & 5.052926 & -0.089017 \\
\hline $\mathrm{C}$ & -2.021113 & 5.747949 & -0.115017 \\
\hline $\mathrm{C}$ & -3.292156 & 7.895924 & -0.342017 \\
\hline $\mathrm{C}$ & -2.053143 & 7.223949 & -0.259017 \\
\hline $\mathrm{C}$ & -3.317184 & 9.303923 & -0.455017 \\
\hline C & -2.116199 & 10.024947 & -0.492017 \\
\hline C & -0.351818 & -9.013017 & -0.844017 \\
\hline C & 0.891194 & -9.654993 & -0.943017 \\
\hline $\mathrm{C}$ & -0.400848 & -7.544018 & -0.634017 \\
\hline C & 0.799137 & -6.804994 & -0.548017 \\
\hline C & -0.491903 & -4.772020 & -0.212017 \\
\hline $\mathrm{C}$ & 0.752110 & -5.412995 & -0.347017 \\
\hline $\mathrm{C}$ & -0.542932 & -3.319021 & 0.045983 \\
\hline $\mathrm{C}$ & 0.652053 & -2.585997 & 0.089983 \\
\hline $\mathrm{C}$ & -0.608986 & -0.637023 & 0.909983 \\
\hline $\mathrm{C}$ & 0.646026 & -1.221997 & 0.325983 \\
\hline $\mathrm{C}$ & -0.659017 & 0.937976 & 0.960983 \\
\hline $\mathrm{C}$ & 0.572969 & 1.614001 & 0.409983 \\
\hline C & -0.736071 & 3.634975 & 0.188983 \\
\hline $\mathrm{C}$ & 0.505942 & 2.981000 & 0.220983 \\
\hline C & -0.770100 & 5.093974 & -0.016017 \\
\hline $\mathrm{C}$ & 0.449886 & 5.802999 & -0.121017 \\
\hline C & -0.845157 & 7.952973 & -0.312017 \\
\hline $\mathrm{C}$ & 0.398856 & 7.283998 & -0.259017 \\
\hline $\mathrm{C}$ & -0.883185 & 9.360972 & -0.429017 \\
\hline $\mathrm{C}$ & 0.311800 & 10.087996 & -0.487017 \\
\hline $\mathrm{C}$ & 2.088180 & -8.924969 & -0.867017 \\
\hline $\mathrm{C}$ & 3.325193 & -9.568944 & -0.978017 \\
\hline C & 2.047150 & -7.449970 & -0.675017 \\
\hline $\mathrm{C}$ & 3.206136 & -6.739946 & -0.632017 \\
\hline $\mathrm{C}$ & 1.957095 & -4.674971 & -0.310017 \\
\hline C & 3.190108 & -5.340947 & -0.463017 \\
\hline C & 1.941067 & -3.276972 & -0.133017 \\
\hline $\mathrm{C}$ & 3.138052 & -2.543948 & -0.142017 \\
\hline $\mathrm{C}$ & 1.815011 & -0.453974 & 0.225983 \\
\hline $\mathrm{C}$ & 3.107024 & -1.140948 & -0.001017 \\
\hline $\mathrm{C}$ & 1.779983 & 0.928025 & 0.266983 \\
\hline $\mathrm{C}$ & 3.023967 & 1.711050 & 0.059983 \\
\hline $\mathrm{C}$ & 1.753927 & 3.759025 & 0.019983 \\
\hline $\mathrm{C}$ & 2.957939 & 3.116049 & -0.030017 \\
\hline $\mathrm{C}$ & 1.658899 & 5.164023 & -0.107017 \\
\hline $\mathrm{C}$ & 2.911883 & 5.956048 & -0.230017 \\
\hline $\mathrm{C}$ & 1.597841 & 8.022022 & -0.340017 \\
\hline C & 2.846855 & 7.360046 & -0.326017 \\
\hline $\mathrm{C}$ & 1.548813 & 9.431021 & -0.448017 \\
\hline $\mathrm{C}$ & 2.736799 & 10.167044 & -0.524017 \\
\hline C & 4.525178 & -8.841920 & -0.928017 \\
\hline $\mathrm{C}$ & 5.749191 & -9.507896 & -1.042017 \\
\hline
\end{tabular}




\begin{tabular}{|c|c|c|c|}
\hline C & 4.508150 & -7.439920 & -0.770017 \\
\hline C & 5.719136 & -6.706896 & -0.744017 \\
\hline $\mathrm{C}$ & 4.397094 & -4.605923 & -0.451017 \\
\hline C & 5.705107 & -5.300896 & -0.600017 \\
\hline C & 4.363066 & -3.209923 & -0.303017 \\
\hline C & 5.619050 & -2.431898 & -0.327017 \\
\hline $\mathrm{C}$ & 4.289009 & -0.383925 & -0.087017 \\
\hline C & 5.583023 & -1.083899 & -0.238017 \\
\hline $\mathrm{C}$ & 4.254981 & 1.027075 & -0.066017 \\
\hline $\mathrm{C}$ & 5.519966 & 1.805100 & -0.199017 \\
\hline C & 4.218924 & 3.892074 & -0.178017 \\
\hline C & 5.464937 & 3.222099 & -0.234017 \\
\hline C & 4.161896 & 5.298073 & -0.251017 \\
\hline $\mathrm{C}$ & 5.353881 & 6.047096 & -0.350017 \\
\hline $\mathrm{C}$ & 4.037840 & 8.110070 & -0.418017 \\
\hline $\mathrm{C}$ & 5.290853 & 7.455095 & -0.425017 \\
\hline $\mathrm{C}$ & 3.976812 & 9.518069 & -0.510017 \\
\hline $\mathrm{C}$ & 5.157797 & 10.261093 & -0.594017 \\
\hline C & 6.955177 & -8.794872 & -1.005017 \\
\hline C & 8.165191 & -9.479847 & -1.113017 \\
\hline $\mathrm{C}$ & 6.948149 & -7.390872 & -0.864017 \\
\hline C & 8.166135 & -6.677847 & -0.839017 \\
\hline $\mathrm{C}$ & 6.921093 & -4.565872 & -0.600017 \\
\hline $\mathrm{C}$ & 8.152107 & -5.258848 & -0.723017 \\
\hline $\mathrm{C}$ & 6.907064 & -3.148872 & -0.473017 \\
\hline $\mathrm{C}$ & 8.099049 & -2.393849 & -0.510017 \\
\hline $\mathrm{C}$ & 6.830007 & -0.287874 & -0.305017 \\
\hline $\mathrm{C}$ & 8.061021 & -0.971849 & -0.428017 \\
\hline $\mathrm{C}$ & 6.767979 & 1.134125 & -0.288017 \\
\hline C & 7.957964 & 1.897148 & -0.354017 \\
\hline $\mathrm{C}$ & 6.658922 & 3.976123 & -0.326017 \\
\hline $\mathrm{C}$ & 7.905935 & 3.317147 & -0.358017 \\
\hline $\mathrm{C}$ & 6.605894 & 5.390121 & -0.374017 \\
\hline C & 7.798879 & 6.148145 & -0.460017 \\
\hline C & 6.476838 & 8.212119 & -0.516017 \\
\hline $\mathrm{C}$ & 7.733851 & 7.568144 & -0.533017 \\
\hline $\mathrm{C}$ & 6.402810 & 9.619117 & -0.596017 \\
\hline $\mathrm{C}$ & 7.570794 & 10.377141 & -0.679017 \\
\hline $\mathrm{C}$ & 9.391177 & -8.802823 & -1.080017 \\
\hline $\mathrm{C}$ & 10.550193 & -9.581800 & -1.213017 \\
\hline $\mathrm{C}$ & 9.400149 & -7.383823 & -0.931017 \\
\hline C & 10.595134 & -6.645799 & -0.851017 \\
\hline $\mathrm{C}$ & 9.371092 & -4.543823 & -0.719017 \\
\hline C & 10.580107 & -5.260799 & -0.741017 \\
\hline $\mathrm{C}$ & 9.340062 & -3.047824 & -0.668017 \\
\hline $\mathrm{C}$ & 10.501047 & -2.266801 & -0.830017 \\
\hline $\mathrm{C}$ & 9.263006 & -0.239825 & -0.509017 \\
\hline $\mathrm{C}$ & 10.464020 & -0.932801 & -0.756017 \\
\hline $\mathrm{C}$ & 9.211977 & 1.250174 & -0.402017 \\
\hline $\mathrm{C}$ & 10.378961 & 2.028197 & -0.314017 \\
\hline $\mathrm{C}$ & 9.110920 & 4.068172 & -0.392017 \\
\hline C & 10.332934 & 3.363196 & -0.309017 \\
\hline $\mathrm{C}$ & 9.055892 & 5.491170 & -0.480017 \\
\hline $\mathrm{C}$ & 10.216876 & 6.282194 & -0.622017 \\
\hline C & 8.921835 & 8.345168 & -0.633017 \\
\hline C & 10.150849 & 7.668192 & -0.689017 \\
\hline $\mathrm{C}$ & 8.832807 & 9.769166 & -0.693017 \\
\hline $\mathrm{C}$ & 9.945790 & 10.620188 & -0.758017 \\
\hline $\mathrm{H}$ & -10.955798 & -10.048229 & -1.328017 \\
\hline $\mathrm{H}$ & -8.751776 & -11.131185 & -1.336017 \\
\hline $\mathrm{H}$ & -11.165846 & -7.654233 & -1.072017 \\
\hline $\mathrm{H}$ & -11.264887 & -5.600235 & -0.674017 \\
\hline $\mathrm{H}$ & -11.357932 & -3.303237 & -0.492017 \\
\hline $\mathrm{H}$ & -11.380974 & -1.238238 & -0.888017 \\
\hline $\mathrm{H}$ & -11.433020 & 1.064761 & -0.842017 \\
\hline $\mathrm{H}$ & -11.512060 & 3.083760 & -0.291017 \\
\hline $\mathrm{H}$ & -11.560106 & 5.372759 & -0.357017 \\
\hline $\mathrm{H}$ & -11.586146 & 7.409758 & -0.696017 \\
\hline $\mathrm{H}$ & -11.517194 & 9.807760 & -0.831017 \\
\hline $\mathrm{H}$ & -9.386218 & 11.023802 & -0.795017 \\
\hline $\mathrm{H}$ & -6.341779 & -10.988137 & -1.219017 \\
\hline
\end{tabular}




$$
\begin{array}{rrr}
-6.971219 & 11.025850 & -0.694017 \\
-3.940781 & -10.885089 & -1.138017 \\
-4.558219 & 11.056899 & -0.621017 \\
-1.512783 & -10.809041 & -1.101017 \\
-2.140220 & 11.105947 & -0.581017 \\
0.927216 & -10.728992 & -1.093017 \\
0.279779 & 11.168995 & -0.574017 \\
3.356214 & -10.644943 & -1.111017 \\
2.695777 & 11.248043 & -0.602017 \\
5.764213 & -10.585895 & -1.160017 \\
5.107775 & 11.342092 & -0.661017 \\
8.151212 & -10.558848 & -1.228017 \\
7.493773 & 11.458139 & -0.732017 \\
11.549185 & -9.181780 & -1.236017 \\
10.485214 & -10.658801 & -1.322017 \\
11.564144 & -7.116780 & -0.862017 \\
11.538097 & -4.772780 & -0.657017 \\
11.458056 & -2.713782 & -1.048017 \\
11.393010 & -0.410783 & -0.924017 \\
11.353970 & 1.574216 & -0.228017 \\
11.281924 & 3.864215 & -0.218017 \\
11.202885 & 5.860213 & -0.717017 \\
11.087838 & 8.188211 & -0.805017 \\
10.967796 & 10.283209 & -0.746017 \\
9.819768 & 11.695186 & -0.806017 \\
-0.658024 & 1.287976 & 2.380983 \\
-0.435977 & -1.051019 & 2.367983 \\
-1.116954 & -2.214033 & 3.280983 \\
-0.349042 & 2.207983 & 2.442983 \\
-0.285956 & -2.134016 & 4.477983 \\
-2.584960 & -1.946062 & 3.370983 \\
-0.890937 & -3.082028 & 2.659983 \\
-3.461938 & -3.012080 & 3.166983 \\
-4.837942 & -2.809107 & 3.212983 \\
-5.341968 & -1.532117 & 3.447983 \\
-4.468989 & -0.467100 & 3.659983 \\
-3.092985 & -0.672072 & 3.632983 \\
-3.063919 & -3.999072 & 2.941983 \\
-5.510926 & -3.639120 & 3.030983 \\
-6.413971 & -1.363138 & 3.448983 \\
-4.859009 & 0.531893 & 3.824983 \\
-2.425002 & 0.176941 & 3.749983 \\
0.865059 & -2.859993 & 4.549983 \\
-0.518971 & -1.362021 & 5.206983 \\
1.462058 & -2.837981 & 5.454983 \\
0.950077 & -3.758991 & 3.944983 \\
2.382042 & -2.028963 & 3.359983 \\
1.733046 & -2.227976 & 2.513983 \\
-0.388994 & -0.198018 & 2.877983 \\
2.557016 & -0.707959 & 3.904983 \\
3.442059 & -2.857942 & 3.660983 \\
3.625087 & -4.271938 & 3.208983 \\
3.583101 & -4.977939 & 4.044983 \\
2.832092 & -4.532954 & 2.501983 \\
4.583090 & -4.409919 & 2.698983 \\
\hline 1.524044 & -2.104920 & 4.772983 \\
\hline & -0.613938 & 4.710983 \\
\hline & 0.110027 & 3.716983 \\
\hline
\end{array}
$$

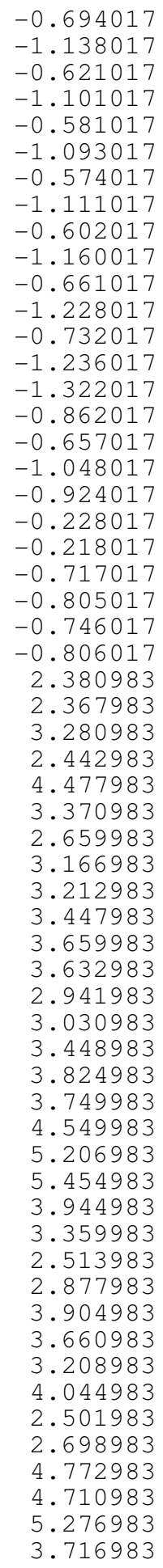

$$
\begin{array}{r}
-7.399000 \\
-8.222123 \\
-6.018740 \\
-5.428247 \\
-3.052926 \\
-3.950329 \\
-1.731158 \\
-1.164676 \\
1.240400
\end{array}
$$

$-1.091745$

$-1.094215$

$-0.963978$

$-0.827673$

$-0.632745$

$-0.688162$

$-0.545263$

$-0.507654$

$-0.645291$ 


\begin{tabular}{|c|c|c|c|}
\hline $\mathrm{C}$ & -9.440119 & 0.319668 & -0.469644 \\
\hline $\mathrm{C}$ & -10.277923 & 2.559912 & -0.608451 \\
\hline $\mathrm{C}$ & -8.994029 & 3.100800 & -0.387753 \\
\hline $\mathrm{C}$ & -9.807955 & 5.505340 & -0.324056 \\
\hline $\mathrm{C}$ & -8.751493 & 4.576152 & -0.334724 \\
\hline $\mathrm{C}$ & -9.588165 & 6.822409 & -0.349326 \\
\hline $\mathrm{C}$ & -8.283455 & 7.361453 & -0.381949 \\
\hline $\mathrm{C}$ & -9.083957 & 9.720998 & -0.586563 \\
\hline $\mathrm{C}$ & -8.041144 & 8.766011 & -0.466551 \\
\hline $\mathrm{C}$ & -8.809162 & 11.084844 & -0.667674 \\
\hline $\mathrm{C}$ & -7.498607 & 11.541730 & 0459 \\
\hline $\mathrm{C}$ & -9.261799 & -7.669355 & -0 . \\
\hline $\mathrm{C}$ & -8.143937 & -8.507895 & 9725 \\
\hline $\mathrm{C}$ & -9.101854 & -6.266034 & -0.839037 \\
\hline $\mathrm{C}$ & -7.802551 & -5.716118 & -0.722330 \\
\hline $\mathrm{C}$ & -8.731323 & -3.435410 & -0.592079 \\
\hline $\mathrm{C}$ & -7.619261 & -4.316402 & -0.595918 \\
\hline $\mathrm{C}$ & -8.547940 & -2.030643 & -0.489835 \\
\hline $\mathrm{C}$ & -7.187986 & -1.463863 & -0.341961 \\
\hline $\mathrm{C}$ & -8.128688 & 0.818429 & -0.309028 \\
\hline $\mathrm{C}$ & -6.993260 & -0.130891 & -0.233537 \\
\hline $\mathrm{C}$ & -7.896435 & 2.220621 & -0 . \\
\hline $\mathrm{C}$ & -6.584558 & 2.740504 & -0.138746 \\
\hline $\mathrm{C}$ & -7.424587 & 5.055211 & -0.275903 \\
\hline $\mathrm{C}$ & -6.343827 & 4.145839 & -0.168946 \\
\hline $\mathrm{C}$ & -7.187205 & 6.457392 & -0.310854 \\
\hline $\mathrm{C}$ & -5.863747 & 6.948074 & -0.275511 \\
\hline $\mathrm{C}$ & -6.702931 & 9.242038 & -0.448883 \\
\hline $\mathrm{C}$ & -5.622311 & 8.337899 & -0.347374 \\
\hline $\mathrm{C}$ & -6.440810 & 10.634006 & -0.535499 \\
\hline $\mathrm{C}$ & -5.124904 & 11.107308 & -0.523033 \\
\hline $\mathrm{C}$ & -6.856279 & -7.978169 & -0.877793 \\
\hline $\mathrm{C}$ & -5.748499 & -8.837164 & -0.910769 \\
\hline $\mathrm{C}$ & -6.680946 & -6.582716 & -0.7 \\
\hline $\mathrm{C}$ & -5.304909 & -6.033558 & -0.616341 \\
\hline $\mathrm{C}$ & -6.235753 & -3.773061 & -0.454770 \\
\hline $\mathrm{C}$ & -5.124369 & -4.644382 & -0.463475 \\
\hline $\mathrm{C}$ & -6.033264 & -2.387044 & -0.305976 \\
\hline $\mathrm{C}$ & -4.737401 & -1.871673 & -0.118677 \\
\hline $\mathrm{C}$ & -5.627099 & 0.407166 & -0.052559 \\
\hline $\mathrm{C}$ & -4.545855 & -0.420040 & 0.015065 \\
\hline $\mathrm{C}$ & -5.437747 & 1.801073 & 0.000141 \\
\hline $\mathrm{C}$ & -4.069803 & 2.414260 & 0.119707 \\
\hline $\mathrm{C}$ & -5.020944 & 4.656439 & -0.089048 \\
\hline C & -3.868801 & 3.733141 & 65 \\
\hline $\mathrm{C}$ & -4.779398 & 6.049917 & -0.161185 \\
\hline $\mathrm{C}$ & -3.453861 & 6.551049 & -0.120648 \\
\hline $\mathrm{C}$ & -4.294861 & 8.829703 & -0.329090 \\
\hline $\mathrm{C}$ & -3.209038 & 7.936694 & -0.220189 \\
\hline $\mathrm{C}$ & -4.052090 & 10.218403 & -0.423667 \\
\hline $\mathrm{C}$ & -2.738663 & 10.705855 & -0.425784 \\
\hline $\mathrm{C}$ & -4.447184 & -8.337431 & -0.808768 \\
\hline $\mathrm{C}$ & -3.345391 & -9.202987 & -0.867106 \\
\hline $\mathrm{C}$ & -4.236864 & -6.873513 & -0.646129 \\
\hline $\mathrm{C}$ & -2.922621 & -6.376234 & 524423 \\
\hline $\mathrm{C}$ & -3.821950 & -4.126332 & -0.303653 \\
\hline $\mathrm{C}$ & -2.713600 & -4.998891 & -0.333791 \\
\hline $\mathrm{C}$ & -3.659172 & -2.695641 & -0.093949 \\
\hline $\mathrm{C}$ & -2.296508 & -2.171067 & 0.147471 \\
\hline $\mathrm{C}$ & -3.172832 & 0.091122 & 0.284886 \\
\hline $\mathrm{C}$ & -2.123062 & -0.802930 & 0.403544 \\
\hline $\mathrm{C}$ & -2.941071 & 1.478295 & 0.343209 \\
\hline $\mathrm{C}$ & -1.670423 & 1.990154 & 0.520862 \\
\hline $\mathrm{C}$ & -2.541191 & 4.298174 & 0.135523 \\
\hline $\mathrm{C}$ & -1.415421 & 3.358054 & 0.353828 \\
\hline $\mathrm{C}$ & -2.306750 & 5.618031 & 0.027344 \\
\hline $\mathrm{C}$ & -1.034550 & 6.098535 & 0.022646 \\
\hline $\mathrm{C}$ & -1.882907 & 8.437575 & -0.221989 \\
\hline $\mathrm{C}$ & -0.785433 & 7.557797 & -0.125174 \\
\hline $\mathrm{C}$ & -1.653220 & 9.827504 & -0.334418 \\
\hline $\mathrm{C}$ & -0.341459 & 10.325172 & -0.363226 \\
\hline
\end{tabular}




\begin{tabular}{|c|c|c|c|}
\hline $\mathrm{C}$ & -2.038554 & -8.707742 & -0.777267 \\
\hline $\mathrm{C}$ & -0.936732 & -9.567636 & -0.872754 \\
\hline $\mathrm{C}$ & -1.818280 & -7.250941 & -0.589732 \\
\hline $\mathrm{C}$ & -0.504903 & -6.744229 & -0.503700 \\
\hline $\mathrm{C}$ & -1.430060 & -4.468136 & -0.147844 \\
\hline $\mathrm{C}$ & -0.296508 & -5.366931 & -0.303222 \\
\hline $\mathrm{C}$ & -1.212838 & -3.030456 & 0.109330 \\
\hline $\mathrm{C}$ & 0.102699 & -2.542831 & 0.208783 \\
\hline C & -0.798197 & -0.394404 & 1.024545 \\
\hline $\mathrm{C}$ & 0.350893 & -1.211117 & 0.478065 \\
\hline $\mathrm{C}$ & -0.535650 & 1.156381 & 1.075069 \\
\hline $\mathrm{C}$ & 0.814913 & 1.610621 & 0.553504 \\
\hline $\mathrm{C}$ & -0.118684 & 3.837503 & 0.338792 \\
\hline $\mathrm{C}$ & 0.995438 & 2.972834 & 0.375278 \\
\hline $\mathrm{C}$ & 0.090966 & 5.279993 & 0.151485 \\
\hline $\mathrm{C}$ & 1.439086 & 5.747814 & 0.034068 \\
\hline $\mathrm{C}$ & 0.539493 & 8.065024 & -0.171518 \\
\hline $\mathrm{C}$ & 1.651479 & 7.196133 & -0.113233 \\
\hline $\mathrm{C}$ & 0.755093 & 9.454893 & -0.294101 \\
\hline $\mathrm{C}$ & 2.064930 & 9.957988 & -0.355773 \\
\hline $\mathrm{C}$ & 0.371937 & -9.067076 & -0.815349 \\
\hline C & 1.469076 & -9.926446 & -0.939317 \\
\hline $\mathrm{C}$ & 0.602709 & -7.607967 & -0.632456 \\
\hline $\mathrm{C}$ & 1.870204 & -7.120977 & -0.606145 \\
\hline $\mathrm{C}$ & 1.021950 & -4.863519 & -0.260960 \\
\hline $\mathrm{C}$ & 2.110524 & -5.743564 & -0.436281 \\
\hline $\mathrm{C}$ & 1.238305 & -3.463042 & -0.033428 \\
\hline $\mathrm{C}$ & 2.570198 & -2.987568 & -0.100165 \\
\hline $\mathrm{C}$ & 1.643118 & -0.670434 & 0.362035 \\
\hline $\mathrm{C}$ & 2.788076 & -1.580026 & 0.090791 \\
\hline $\mathrm{C}$ & 1.868308 & 0.692724 & 0.408231 \\
\hline $\mathrm{C}$ & 3.244340 & 1.202087 & 0.199184 \\
\hline $\mathrm{C}$ & 2.363082 & 3.528467 & 0.191546 \\
\hline C & 3.449293 & 2.627381 & 0.096734 \\
\hline $\mathrm{C}$ & 2.533293 & 4.872592 & 0.035956 \\
\hline $\mathrm{C}$ & 3.901216 & 5.423025 & -0.107244 \\
\hline $\mathrm{C}$ & 2.966853 & 7.697683 & -0.207568 \\
\hline $\mathrm{C}$ & 4.081139 & 6.817941 & -0.206481 \\
\hline $\mathrm{C}$ & 3.168900 & 9.090430 & -0.322010 \\
\hline $\mathrm{C}$ & 4.471876 & 9.598550 & -0.413170 \\
\hline $\mathrm{C}$ & 2.780646 & -9.429221 & -0.919135 \\
\hline $\mathrm{C}$ & 3.860421 & -10.305357 & -1.060394 \\
\hline $\mathrm{C}$ & 3.020633 & -8.046552 & -0.767130 \\
\hline $\mathrm{C}$ & 4.344317 & -7.545106 & -0.775089 \\
\hline $\mathrm{C}$ & 3.430708 & -5.240248 & -0.449297 \\
\hline $\mathrm{C}$ & 4.587297 & -6.160878 & -0.633443 \\
\hline $\mathrm{C}$ & 3.651491 & -3.862524 & -0.298212 \\
\hline $\mathrm{C}$ & 5.027738 & -3.326379 & -0.349579 \\
\hline $\mathrm{C}$ & 4.096621 & -1.081016 & -0.052104 \\
\hline $\mathrm{C}$ & 5.238667 & -1.997256 & -0.247759 \\
\hline $\mathrm{C}$ & 4.321723 & 0.305643 & -0.005515 \\
\hline $\mathrm{C}$ & 5.704196 & 0.854863 & -0.164392 \\
\hline $\mathrm{C}$ & 4.817509 & 3.154129 & -0.079884 \\
\hline $\mathrm{C}$ & 5.911012 & 2.263180 & -0.178187 \\
\hline $\mathrm{C}$ & 5.011098 & 4.548186 & -0.151526 \\
\hline $\mathrm{C}$ & 6.313096 & 5.068658 & -0.279292 \\
\hline $\mathrm{C}$ & 5.386179 & 7.338755 & -0.321840 \\
\hline $\mathrm{C}$ & 6.501403 & 6.465851 & -0.352998 \\
\hline $\mathrm{C}$ & 5.577224 & 8.734497 & -0.415550 \\
\hline $\mathrm{C}$ & 6.872871 & 9.250178 & -0.521202 \\
\hline $\mathrm{C}$ & 5.174937 & -9.821909 & -1.063369 \\
\hline $\mathrm{C}$ & 6.237962 & -10.714024 & -1.208008 \\
\hline $\mathrm{C}$ & 5.425183 & -8.439496 & -0.928086 \\
\hline $\mathrm{C}$ & 6.752553 & -7.958088 & -0.944085 \\
\hline $\mathrm{C}$ & 5.915735 & -5.657366 & -0.666510 \\
\hline $\mathrm{C}$ & 6.997548 & -6.561005 & -0.827236 \\
\hline $\mathrm{C}$ & 6.161160 & -4.262800 & -0.535006 \\
\hline C & 7.469183 & -3.735236 & -0.599484 \\
\hline $\mathrm{C}$ & 6.608485 & -1.438054 & -0.336649 \\
\hline $\mathrm{C}$ & 7.691170 & -2.330807 & -0.502568 \\
\hline $\mathrm{C}$ & 6.807006 & -0.028615 & -0.298157 \\
\hline
\end{tabular}




\begin{tabular}{|c|c|c|c|}
\hline C & 8.114269 & 0.508050 & -0.389639 \\
\hline C & 7.218998 & 2.789835 & -0.299276 \\
\hline $\mathrm{C}$ & 8.321979 & 1.914948 & -0.375830 \\
\hline $\mathrm{C}$ & 7.422619 & 4.192947 & -0.337098 \\
\hline C & 8.730364 & 4.722368 & -0.451571 \\
\hline C & 7.802452 & 6.995496 & -0.470531 \\
\hline C & 8.921232 & 6.133014 & -0.517236 \\
\hline C & 7.981543 & 8.392768 & -0.548404 \\
\hline $\mathrm{C}$ & 9.266196 & 8.925618 & -0.657350 \\
\hline $\mathrm{C}$ & 7.566338 & -10.268365 & -1.220039 \\
\hline $\mathrm{C}$ & 8.563129 & -11.241604 & -1.387602 \\
\hline C & 7.834962 & -8.874509 & -1.077083 \\
\hline $\mathrm{C}$ & 9.144874 & -8.363308 & -1.046917 \\
\hline $\mathrm{C}$ & 8.325386 & -6.076843 & -0.861743 \\
\hline $\mathrm{C}$ & 9.382947 & -7.000057 & -0.935686 \\
\hline $\mathrm{C}$ & 8.566182 & -4.601287 & -0.795772 \\
\hline C & 9.846512 & -4.041425 & -0.971737 \\
\hline C & 9.004255 & -1.827516 & -0.607153 \\
\hline C & 10.054126 & -2.724614 & -0.884145 \\
\hline C & 9.228182 & -0.354518 & -0.486234 \\
\hline C & 10.519144 & 0.198968 & -0.439338 \\
\hline C & 9.641963 & 2.436381 & -0.440962 \\
\hline C & 10.716866 & 1.518824 & -0.418388 \\
\hline C & 9.845885 & 3.847835 & -0.507204 \\
\hline C & 11.127941 & 4.418616 & -0.659823 \\
\hline $\mathrm{C}$ & 10.227564 & 6.681945 & -0.640941 \\
\hline $\mathrm{C}$ & 11.313164 & 5.793810 & -0.718212 \\
\hline $\mathrm{C}$ & 10.396771 & 8.098098 & -0.699703 \\
\hline C & 11.645344 & 8.731219 & -0.795325 \\
\hline $\mathrm{H}$ & -12.648400 & -7.833217 & -1.193731 \\
\hline $\mathrm{H}$ & -10.676687 & -9.292650 & -1.197315 \\
\hline $\mathrm{H}$ & -12.428044 & -5.440344 & -0.982567 \\
\hline $\mathrm{H}$ & -12.154336 & -3.394460 & -0.654120 \\
\hline $\mathrm{H}$ & -11.838738 & -1.122749 & -0.492106 \\
\hline $\mathrm{H}$ & -11.499149 & 0.918561 & -0.842629 \\
\hline $\mathrm{H}$ & -11.136887 & 3.186867 & -0.782182 \\
\hline $\mathrm{H}$ & -10.838154 & 5.191942 & -0.281705 \\
\hline $\mathrm{H}$ & -10.468298 & 7.442059 & -0.333860 \\
\hline $\mathrm{H}$ & -10.124885 & 9.453368 & -0.635305 \\
\hline $\mathrm{H}$ & -9.622498 & 11.794374 & -0.758414 \\
\hline $\mathrm{H}$ & -7.311355 & 12.607205 & -0.708111 \\
\hline $\mathrm{H}$ & -8.277637 & -9.579547 & $-1.0 \varsigma$ \\
\hline $\mathrm{H}$ & -4.935146 & 12.173370 & -0.594735 \\
\hline $\mathrm{H}$ & -5.901277 & -9.905185 & -1.025093 \\
\hline $\mathrm{H}$ & -2.560531 & 11.773050 & -0.507255 \\
\hline $\mathrm{H}$ & -3.506276 & -10.267819 & -0.996788 \\
\hline $\mathrm{H}$ & -0.173478 & 11.393019 & -0.454636 \\
\hline $\mathrm{H}$ & -1.098654 & -10.631400 & -1.009654 \\
\hline $\mathrm{H}$ & 2.225266 & 11.026880 & -0.447968 \\
\hline $\mathrm{H}$ & 1.300789 & -10.990705 & -1.066578 \\
\hline $\mathrm{H}$ & 4.625820 & 10.669224 & -0.495392 \\
\hline $\mathrm{H}$ & 3.676797 & -11.368901 & -1.173 \\
\hline $\mathrm{H}$ & 7.018784 & 10.323205 & -0.589135 \\
\hline $\mathrm{H}$ & 6.024724 & -11.773151 & -1.316407 \\
\hline $\mathrm{H}$ & 9.385522 & 10.003561 & -0.709419 \\
\hline $\mathrm{H}$ & 9.616733 & -11.025799 & -1.442151 \\
\hline $\mathrm{H}$ & 8.301548 & -12.288170 & -1.490995 \\
\hline $\mathrm{H}$ & 10.015227 & -8.996229 & -1.099244 \\
\hline $\mathrm{H}$ & 10.415884 & -6.696752 & -0.895090 \\
\hline $\mathrm{H}$ & 10.705043 & -4.648413 & -1.208392 \\
\hline $\mathrm{H}$ & 11.061000 & -2.383016 & -1.062039 \\
\hline $\mathrm{H}$ & 11.400175 & -0.421688 & -0.404655 \\
\hline $\mathrm{H}$ & 11.745244 & 1.832999 & -0. \\
\hline $\mathrm{H}$ & 12.022678 & 3.830237 & -0 \\
\hline $\mathrm{H}$ & 12.328513 & 6.132140 & -0.843027 \\
\hline $\mathrm{H}$ & 12.588137 & 8.211351 & -0.814789 \\
\hline $\mathrm{H}$ & 11.714547 & 9.811725 & -0.841744 \\
\hline 0 & -0.499397 & 1.484953 & 2.491465 \\
\hline 0 & -0.910399 & -0.881878 & 2.378300 \\
\hline C & -0.590875 & -4.336998 & 3.622030 \\
\hline $\mathrm{H}$ & -0.502070 & 2.456424 & 2.523261 \\
\hline
\end{tabular}




$\begin{array}{rr}\mathrm{C} & -0.106382 \\ \mathrm{C} & -2.003501 \\ \mathrm{H} & 0.103995 \\ \mathrm{C} & -2.407607 \\ \mathrm{C} & -3.742929 \\ \mathrm{C} & -4.693978 \\ \mathrm{C} & -4.298258 \\ \mathrm{C} & -2.964654 \\ \mathrm{H} & -1.666286 \\ \mathrm{H} & -4.037879 \\ \mathrm{H} & -5.734507 \\ \mathrm{H} & -5.030646 \\ \mathrm{H} & -2.652111 \\ \mathrm{C} & 1.356126 \\ \mathrm{H} & -0.771168 \\ \mathrm{H} & 1.532308 \\ \mathrm{H} & 1.851143 \\ \mathrm{C} & 2.045414 \\ \mathrm{C} & 4.089320 \\ \mathrm{C} & 2.873445 \\ \mathrm{H} & 1.705300 \\ \mathrm{H} & -1.502916 \\ \mathrm{~S} & 3.864046 \\ \mathrm{C} & 1.771419 \\ \mathrm{H} & 0.747300 \\ \mathrm{C} & 5.439760 \\ \mathrm{H} & 5.545797 \\ \mathrm{H} & 5.531512 \\ \mathrm{H} & 6.249163 \\ \mathrm{H} & 2.847886 \\ & \end{array}$

\section{RX3}

$\begin{array}{lr}\text { C } & -11.287711 \\ \text { C } & -10.133015 \\ \text { C } & -11.204933 \\ \text { C } & -9.953528 \\ \text { C } & -10.945825 \\ \text { C } & -9.821814 \\ \text { C } & -10.819781 \\ \text { C } & -9.557574 \\ \text { C } & -10.485483 \\ \text { C } & -9.398787 \\ \text { C } & -10.327130 \\ \text { C } & -9.065404 \\ C & -9.969812 \\ \text { C } & -8.878832 \\ \text { C } & -9.800663 \\ \text { C } & -8.517521 \\ \text { C } & -9.409488 \\ \text { C } & -8.329721 \\ \text { C } & -9.188796 \\ \text { C } & -7.895924 \\ \text { C } & -8.877819 \\ \text { C } & -7.725400 \\ \text { C } & -8.777886 \\ \text { C } & -7.502818 \\ \text { C } & -8.529050 \\ \text { C } & -7.379578 \\ \text { C } & -8.406208 \\ \text { C } & -7.071594 \\ \text { C } & -8.109215 \\ \text { C } & -6.934982 \\ \text { C } & -7.933849 \\ \text { C } & -6.643364 \\ \text { C } & -7.570308 \\ \text { C } & -6.454899 \\ \text { C } & -7.386493 \\ \text { C } & -6.081659 \\ \text { C } & -7.010489 \\ \text { C } & -5.894462 \\ & \end{array}$

$$
\begin{array}{rl}
-3.243843 & 4.221771 \\
-4.613725 & 3.315581 \\
-5.140987 & 3.370362 \\
-5.942083 & 3.137645 \\
-6.256769 & 2.906186 \\
-5.243081 & 2.838908 \\
-3.913254 & 2.982676 \\
-3.599505 & 3.211896 \\
-6.735720 & 3.187491 \\
-7.291504 & 2.766012 \\
-5.483153 & 2.645745 \\
-3.116516 & 2.890689 \\
-2.561800 & 3.262570 \\
-3.076918 & 4.517084 \\
-2.435741 & 4.514821 \\
-2.694077 & 5.527096 \\
-4.049866 & 4.436921 \\
-2.128727 & 3.490541 \\
-0.612233 & 3.620493 \\
0.116675 & 3.659653 \\
-2.453909 & 2.496433 \\
-0.249798 & 2.821697 \\
-2.279513 & 3.515294 \\
-0.676816 & 3.585839 \\
-0.312171 & 3.524897 \\
0.005653 & 3.639703 \\
0.649614 & 2.758117 \\
0.656127 & 4.516448 \\
-0.724362 & 3.641508 \\
1.200529 & 3.696624
\end{array}
$$

$$
\begin{array}{rr}
-7.983352 & -1.071278 \\
-8.757425 & -1.08354 \\
-6.599267 & -0.92746 \\
-5.956269 & -0.78225 \\
-3.624294 & -0.53382 \\
-4.472445 & -0.62255 \\
-2.295159 & -0.437729 \\
-1.672833 & -0.42340 \\
0.697473 & -0.56802 \\
-0.178806 & -0.38691 \\
2.026555 & -0.54181 \\
2.620997 & -0.32624 \\
4.994299 & -0.27956 \\
4.106186 & -0.29544 \\
6.318775 & -0.339331 \\
6.905320 & -0.416652 \\
9.224466 & -0.69431 \\
8.314734 & -0.54953 \\
10.595450 & -0.817422 \\
11.102740 & -0.81099 \\
-8.151500 & -0.958696 \\
-8.941811 & -0.990470 \\
-6.743989 & -0.80914 \\
-6.140092 & -0.695022 \\
-3.901456 & -0.53777 \\
-4.734158 & -0.559496 \\
-2.490246 & -0.427086 \\
-1.865443 & -0.291456 \\
0.375222 & -0.233559 \\
-0.525690 & -0.170870 \\
1.786777 & -0.200433 \\
2.361058 & -0.083536 \\
4.637374 & -0.26404 \\
3.773345 & -0.14583 \\
6.046014 & -0.337368 \\
6.587408 & -0.33289 \\
8.841728 & -0.559982 \\
7.982937 & -0.44579 \\
&
\end{array}
$$




\begin{tabular}{|c|c|c|c|}
\hline C & -6.803179 & 10.239636 & -0.688669 \\
\hline $\mathrm{C}$ & -5.506558 & 10.763857 & -0.701322 \\
\hline $\mathrm{C}$ & -6.461060 & -8.359228 & -0.879638 \\
\hline C & -5.318067 & -9.170993 & -0.927880 \\
\hline C & -6.344896 & -6.958621 & -0.728363 \\
\hline C & -4.992705 & -6.353186 & -0.607874 \\
\hline C & -6.019910 & -4.132534 & -0.429209 \\
\hline $\mathrm{C}$ & -4.871783 & -4.957671 & -0.447925 \\
\hline C & -5.877178 & -2.737748 & -0.279803 \\
\hline C & -4.604559 & -2.165770 & -0.104352 \\
\hline $\mathrm{C}$ & -5.594346 & 0.070823 & 0.005436 \\
\hline $\mathrm{C}$ & -4.464809 & -0.770090 & 0.083382 \\
\hline $\mathrm{C}$ & -5.462587 & 1.470754 & 0.066756 \\
\hline $\mathrm{C}$ & -4.124718 & 2.075725 & 0.217135 \\
\hline $\mathrm{C}$ & -5.150257 & 4.335743 & -0.092169 \\
\hline $\mathrm{C}$ & -3.961923 & 3.461349 & 0.091859 \\
\hline $\mathrm{C}$ & -4.961885 & 5.734809 & -0.207953 \\
\hline C & -3.655022 & 6.287160 & -0.201566 \\
\hline $\mathrm{C}$ & -4.586438 & 8.525816 & -0.457092 \\
\hline C & -3.464984 & 7.678648 & -0.339868 \\
\hline C & -4.398888 & 9.920165 & -0.589825 \\
\hline $\mathrm{C}$ & -3.105607 & 10.458807 & -0.616895 \\
\hline $\mathrm{C}$ & -4.037827 & -8.618730 & -0.828888 \\
\hline $\mathrm{C}$ & -2.900220 & -9.438320 & -0.897455 \\
\hline $\mathrm{C}$ & -3.889269 & -7.148159 & -0.655225 \\
\hline $\mathrm{C}$ & -2.595541 & -6.597676 & -0.547498 \\
\hline $\mathrm{C}$ & -3.591730 & -4.384616 & -0.308047 \\
\hline $\mathrm{C}$ & -2.444145 & -5.212721 & -0.362998 \\
\hline C & -3.464660 & -2.996376 & -0.123387 \\
\hline $\mathrm{C}$ & -2.122445 & -2.412685 & 0.083053 \\
\hline $\mathrm{C}$ & -3.120845 & -0.188757 & 0.351276 \\
\hline C & -2.011480 & -1.059770 & 0.391743 \\
\hline $\mathrm{C}$ & -2.957599 & 1.185106 & 0.427242 \\
\hline $\mathrm{C}$ & -1.683421 & 1.777974 & 0.533098 \\
\hline $\mathrm{C}$ & -2.667028 & 4.016505 & 0.111077 \\
\hline $\mathrm{C}$ & -1.491207 & 3.135059 & 0.300687 \\
\hline $\mathrm{C}$ & -2.469655 & 5.402711 & -0.053487 \\
\hline $\mathrm{C}$ & -1.216500 & 5.933664 & -0.090248 \\
\hline $\mathrm{C}$ & -2.158756 & 8.231328 & -0.369932 \\
\hline $\mathrm{C}$ & -1.025872 & 7.398600 & -0.265416 \\
\hline $\mathrm{C}$ & -1.985034 & 9.626598 & -0.514977 \\
\hline $\mathrm{C}$ & -0.693708 & 10.175952 & -0.562747 \\
\hline $\mathrm{C}$ & -1.613284 & -8.891138 & -0.808599 \\
\hline $\mathrm{C}$ & -0.475459 & -9.706807 & -0.892098 \\
\hline $\mathrm{C}$ & -1.453492 & -7.426264 & -0.625839 \\
\hline $\mathrm{C}$ & -0.159634 & -6.867664 & -0.542558 \\
\hline $\mathrm{C}$ & -1.154127 & -4.669328 & -0.243183 \\
\hline $\mathrm{C}$ & -0.007560 & -5.483042 & -0.356746 \\
\hline $\mathrm{C}$ & -0.992997 & -3.225631 & 0.000446 \\
\hline $\mathrm{C}$ & 0.291111 & -2.688566 & 0.098671 \\
\hline $\mathrm{C}$ & -0.695275 & -0.569224 & 0.757976 \\
\hline $\mathrm{C}$ & 0.477624 & -1.346941 & 0.396205 \\
\hline $\mathrm{C}$ & -0.508265 & 0.982697 & 0.840694 \\
\hline $\mathrm{C}$ & 0.819166 & 1.506333 & 0.549693 \\
\hline $\mathrm{C}$ & -0.211563 & 3.673541 & 0.248557 \\
\hline $\mathrm{C}$ & 0.934888 & 2.872091 & 0.321614 \\
\hline $\mathrm{C}$ & -0.070664 & 5.119726 & 0.040013 \\
\hline $\mathrm{C}$ & 1.273229 & 5.686163 & -0.075436 \\
\hline $\mathrm{C}$ & 0.278989 & 7.958232 & -0.326171 \\
\hline $\mathrm{C}$ & 1.426772 & 7.137779 & -0.247452 \\
\hline $\mathrm{C}$ & 0.438241 & 9.352890 & -0.477078 \\
\hline $\mathrm{C}$ & 1.727860 & 9.907804 & -0.546415 \\
\hline $\mathrm{C}$ & 0.813375 & -9.155498 & -0.817707 \\
\hline $\mathrm{C}$ & 1.946787 & -9.972706 & -0.905499 \\
\hline C & 0.984154 & -7.687418 & -0.645021 \\
\hline $\mathrm{C}$ & 2.232543 & -7.151746 & -0.589935 \\
\hline $\mathrm{C}$ & 1.291874 & -4.926964 & -0.292421 \\
\hline $\mathrm{C}$ & 2.417157 & -5.765289 & -0.424445 \\
\hline $\mathrm{C}$ & 1.476198 & -3.549177 & -0.095769 \\
\hline $\mathrm{C}$ & 2.764934 & -2.992635 & -0.076109 \\
\hline $\mathrm{C}$ & 1.759291 & -0.744748 & 0.3 \\
\hline
\end{tabular}




\begin{tabular}{|c|c|c|c|}
\hline C & 2.938826 & -1.609383 & 0.099483 \\
\hline C & 1.922950 & 0.620798 & 0.448201 \\
\hline $\mathrm{C}$ & 3.271931 & 1.198121 & 0.241689 \\
\hline $\mathrm{C}$ & 2.281610 & 3.474850 & 0.152768 \\
\hline C & 3.414406 & 2.655782 & 0.119604 \\
\hline C & 2.404975 & 4.858539 & -0.031980 \\
\hline C & 3.751090 & 5.460951 & -0.163535 \\
\hline C & 2.722262 & 7.690947 & -0.338778 \\
\hline C & 3.873364 & 6.858720 & -0.299045 \\
\hline C & 2.867639 & 9.087719 & -0.484267 \\
\hline C & 4.150581 & 9.646735 & -0.574313 \\
\hline C & 3.238667 & -9.425888 & -0.846517 \\
\hline C & 4.355574 & -10.262083 & -0.934771 \\
\hline C & 3.421986 & -8.033898 & -0.700828 \\
\hline $\mathrm{C}$ & 4.726526 & -7.483129 & -0.659333 \\
\hline C & 3.717990 & -5.212211 & -0.390504 \\
\hline $\mathrm{C}$ & 4.913597 & -6.089662 & -0.520665 \\
\hline C & 3.883331 & -3.826701 & -0.233304 \\
\hline C & 5.239369 & -3.238394 & -0.236669 \\
\hline C & 4.216755 & -1.028242 & 0.021916 \\
\hline C & 5.396918 & -1.900936 & -0.136159 \\
\hline C & 4.385306 & 0.368281 & 0.072437 \\
\hline C & 5.745803 & 0.969921 & -0.069206 \\
\hline C & 4.762135 & 3.233316 & -0.051555 \\
\hline C & 5.893607 & 2.385406 & -0.109954 \\
\hline $\mathrm{C}$ & 4.897798 & 4.631869 & -0.161902 \\
\hline $\mathrm{C}$ & 6.178893 & 5.202117 & -0.283548 \\
\hline C & 5.157658 & 7.429908 & -0.408386 \\
\hline C & 6.309363 & 6.603451 & -0.396280 \\
\hline C & 5.291633 & 8.829433 & -0.538965 \\
\hline $\mathrm{C}$ & 6.566856 & 9.395113 & -0.641954 \\
\hline C & 5.651017 & -9.730088 & -0.883929 \\
\hline C & 6.751560 & -10.583847 & -0.965400 \\
\hline C & 5.844968 & -8.337972 & -0.7 \\
\hline $\mathrm{C}$ & 7.153837 & -7.807798 & -0.713246 \\
\hline C & 6.223204 & -5.536749 & -0.501258 \\
\hline $\mathrm{C}$ & 7.342954 & -6.401014 & -0.603905 \\
\hline $\mathrm{C}$ & 6.412322 & -4.132418 & -0.372008 \\
\hline C & 7.701115 & -3.555432 & -0.391354 \\
\hline $\mathrm{C}$ & 6.745147 & -1.289050 & -0.187265 \\
\hline $\mathrm{C}$ & 7.866164 & -2.141136 & -0.306866 \\
\hline C & 6.885866 & 0.128630 & -0.164690 \\
\hline C & 8.171566 & 0.716559 & -0.236792 \\
\hline C & 7.180194 & 2.962429 & -0.222748 \\
\hline C & 8.319975 & 2.131735 & -0 . \\
\hline C & 7.325259 & 4.371722 & -0.296699 \\
\hline $\mathrm{C}$ & 8.611435 & 4.951862 & -0.405795 \\
\hline $\mathrm{C}$ & 7.589100 & 7.183116 & -0.509729 \\
\hline $\mathrm{C}$ & 8.743806 & 6.366864 & -0.513043 \\
\hline $\mathrm{C}$ & 7.710771 & 8.583923 & -0.627831 \\
\hline $\mathrm{C}$ & 8.973820 & 9.166355 & -0.735179 \\
\hline $\mathrm{C}$ & 8.061703 & -10.090419 & -0.915828 \\
\hline C & 9.098049 & -11.030861 & -1.016616 \\
\hline C & 8.273507 & -8.685312 & -0.779691 \\
\hline C & 9.561482 & -8.126092 & -0.689814 \\
\hline C & 8.652594 & -5.867591 & 84031 \\
\hline C & 9.745249 & -6.752232 & -0.588108 \\
\hline C & 8.835972 & -4.381820 & -0.533510 \\
\hline C & 10.099554 & -3.775207 & -0.675926 \\
\hline $\mathrm{C}$ & 9.161320 & -1.587459 & -0.378414 \\
\hline C & 10.253729 & -2.448484 & -0.604459 \\
\hline $\mathrm{C}$ & 9.321973 & -0.102019 & -0.284395 \\
\hline C & 10.587316 & 0.507041 & -0.216654 \\
\hline C & 9.617784 & 2.706277 & -0.307097 \\
\hline $\mathrm{C}$ & 10.729188 & 1.835659 & -0.227801 \\
\hline C & 9.763360 & 4.123071 & -0.416436 \\
\hline C & 11.022 & 4.741890 & -0.573516 \\
\hline C & 10.027587 & 6.966119 & -0.636223 \\
\hline C & 11.150584 & 6.122264 & -0.673286 \\
\hline C & 10.137922 & 8.386323 & -0.736432 \\
\hline $\mathrm{C}$ & 11.358866 & 9.070618 & -0.833695 \\
\hline
\end{tabular}




\begin{tabular}{|c|c|c|c|}
\hline $\mathrm{H}$ & -12.256066 & -8.458952 & -1.179653 \\
\hline $\mathrm{H}$ & -10.219507 & -9.831777 & -1.199419 \\
\hline $\mathrm{H}$ & -12.134966 & -6.056972 & -0.944216 \\
\hline $\mathrm{H}$ & -11.950327 & -4.013543 & -0.533614 \\
\hline $\mathrm{H}$ & -11.730215 & -1.722957 & -0.357369 \\
\hline $\mathrm{H}$ & -11.478932 & 0.328868 & -0.768199 \\
\hline $\mathrm{H}$ & -11.207588 & 2.620552 & -0.725811 \\
\hline $\mathrm{H}$ & -10.984953 & 4.638717 & -0.203077 \\
\hline $\mathrm{H}$ & -10.701576 & 6.907853 & -0.312395 \\
\hline $\mathrm{H}$ & -10.438396 & 8.911961 & -0.735332 \\
\hline $\mathrm{H}$ & -10.030458 & 11.270636 & -0.926076 \\
\hline $\mathrm{H}$ & -7.748351 & 12.172134 & -0.911053 \\
\hline $\mathrm{H}$ & -7.814009 & -10.016448 & -1.108334 \\
\hline $\mathrm{H}$ & -5.359636 & 11.833640 & -0.803209 \\
\hline $\mathrm{H}$ & -5.426756 & -10.242844 & -1.051413 \\
\hline $\mathrm{H}$ & -2.971057 & 11.529564 & -0.724740 \\
\hline $\mathrm{H}$ & -3.017850 & -10.508063 & -1.028497 \\
\hline $\mathrm{H}$ & -0.569791 & 11.247175 & -0.677158 \\
\hline $\mathrm{H}$ & -0.593742 & -10.776606 & -1.022287 \\
\hline $\mathrm{H}$ & 1.844486 & 10.979698 & -0.661312 \\
\hline $\mathrm{H}$ & 1.822956 & -11.043278 & -1.026069 \\
\hline $\mathrm{H}$ & 4.261250 & 10.719989 & -0.682828 \\
\hline $\mathrm{H}$ & 4.216198 & -11.332378 & -1.043491 \\
\hline $\mathrm{H}$ & 6.669281 & 10.470432 & -0.740091 \\
\hline $\mathrm{H}$ & 6.583141 & -11.650816 & -1.069824 \\
\hline $\mathrm{H}$ & 9.049773 & 10.245553 & -0.818933 \\
\hline $\mathrm{H}$ & 10.145202 & -10.780512 & -1.021017 \\
\hline $\mathrm{H}$ & 8.879823 & -12.087608 & -1.116480 \\
\hline $\mathrm{H}$ & 10.452924 & -8.731486 & -0.686540 \\
\hline $\mathrm{H}$ & 10.762329 & -6.407241 & -0.496192 \\
\hline $\mathrm{H}$ & 10.985574 & -4.355480 & -0.878263 \\
\hline $\mathrm{H}$ & 11.251168 & -2.068431 & -0.757961 \\
\hline $\mathrm{H}$ & 11.490890 & -0.076921 & -0.140267 \\
\hline $\mathrm{H}$ & 11.740887 & 2.198015 & -0.157280 \\
\hline $\mathrm{H}$ & 11.940335 & 4.185454 & -0.656337 \\
\hline $\mathrm{H}$ & 12.150766 & 6.502878 & -0.799747 \\
\hline $\mathrm{H}$ & 12.323729 & 8.593231 & -0.823316 \\
\hline $\mathrm{H}$ & 11.383846 & 10.151100 & -0.912203 \\
\hline 0 & -0.607542 & 0.143598 & 1.984353 \\
\hline $\mathrm{C}$ & -1.547982 & -2.871165 & 3.525908 \\
\hline $\mathrm{C}$ & -0.725797 & -2.085074 & 4.229435 \\
\hline $\mathrm{C}$ & -2.995174 & -2.658365 & 3.364349 \\
\hline $\mathrm{H}$ & -1.148796 & -3.779419 & 3.070095 \\
\hline $\mathrm{C}$ & -3.823512 & -3.763321 & 3.136271 \\
\hline $\mathrm{C}$ & -5.201904 & -3.608418 & 3.035234 \\
\hline C & -5.769631 & -2.341736 & 3.153834 \\
\hline $\mathrm{C}$ & -4.950084 & -1.231383 & 3.353025 \\
\hline $\mathrm{C}$ & -3.572050 & -1.385680 & 3.448956 \\
\hline $\mathrm{H}$ & -3.378725 & -4.749843 & 3.032192 \\
\hline $\mathrm{H}$ & -5.829983 & -4.472470 & 2.845775 \\
\hline $\mathrm{H}$ & -6.842597 & -2.214271 & 3.056306 \\
\hline $\mathrm{H}$ & -5.382140 & -0.236253 & 3.396535 \\
\hline $\mathrm{H}$ & -2.930788 & -0.511346 & 3.528619 \\
\hline $\mathrm{C}$ & 0.731791 & -2.394581 & $4.41^{\circ}$ \\
\hline $\mathrm{H}$ & -1.116090 & -1.193788 & 4.717055 \\
\hline $\mathrm{H}$ & 1.026974 & -2.327198 & 5.469557 \\
\hline $\mathrm{H}$ & 0.934222 & -3.414540 & 4.074635 \\
\hline $\mathrm{C}$ & 1.610566 & -1.443586 & 3.580015 \\
\hline $\mathrm{C}$ & 3.879096 & -0.310416 & 3.596174 \\
\hline $\mathrm{C}$ & 2.821554 & 0.612851 & 3.805246 \\
\hline $\mathrm{H}$ & 1.235070 & -1.494206 & 2.534592 \\
\hline S & 3.364755 & -1.910006 & 3.476319 \\
\hline $\mathrm{C}$ & 1.599362 & 0.007332 & 3.840284 \\
\hline $\mathrm{H}$ & 0.655134 & 0.536637 & 3.936922 \\
\hline $\mathrm{H}$ & 2.991889 & 1.681104 & 3.881405 \\
\hline $\mathrm{C}$ & 5.316833 & 0.060333 & 3.478276 \\
\hline $\mathrm{H}$ & 5.902874 & -0.713017 & 2.979151 \\
\hline $\mathrm{H}$ & 5.409988 & 1.001389 & 2.927699 \\
\hline $\mathrm{H}$ & 5.725412 & 0.221849 & 4.48356 \\
\hline
\end{tabular}


TS3

C $\quad-11.945571$

C $\quad-10.868520$

C $\quad-11.736651$

C $\quad-10.432682$

C $\quad-11.208823$

C $\quad-10.166767$

C $\quad-10.961899$

C $\quad-9.648929$

$-10.354072$

$-9.354015$

$-10.076148$

$-8.767176$

$-9.453319$

$-8.447262$

$-9.164396$

$-7.833423$

$-8.511562$

$-7.519504$

$-8.167641$

$-6.834664$

$-9.563548$

$-8.489496$

$-9.335629$

$-8.010658$

$-8.827793$

$-7.759739$

$-8.577875$

$-7.193904$

$-8.022040$

$-6.935981$

$-7.718122$

$-6.382148$

$-7.096286$

$-6.065229$

$-6.785367$

$-5.437391$

$-6.157528$

$-5.124472$

$-5.824608$

$-4.486631$

$-7.177523$

$-6.114469$

$-6.933604$

$-5.531632$

$-6.351766$

$-5.283712$

$-6.083847$

$-4.764873$

$-5.548009$

$-4.502954$

$-5.289090$

$-3.903118$

$-4.715255$

$-3.612198$

$-4.400336$

$-3.049361$

$-3.773496$

$-2.733441$

$-3.460577$

$-2.124601$

$-4.789494$

$-3.733440$

$-4.506579$

$-3.168604$

$-3.956739$

$-2.890684$

$-3.706819$

$-2.316846$

$-3.108981$
$-6.927713$

$-7.805650$

$-5.559701$

$-5.037625$

$-2.628670$

$-3.575609$

$-1.319656$

$-0.817579$

1.624380

0.653439

2.931396

3. 407473

5.851433

4.868492

7.152450

7. 618528

10.002488

9.002546

11.344508

11.733586

$-7.320574$

$-8.214511$

$-5.930560$

$-5.448483$

$-3.128531$

$-4.062468$

$-1.736516$

$-1.239435$

1. 085517

0.081580

2. 474534

2. 929613

5.278571

4.317631

6.662589

7.082668

9.407626

8.453686

10.777645

11.181724

$-7.752434$

$-8.665372$

$-6.369420$

$-5.892338$

$-3.589386$

$-4.514323$

$-2.217370$

$-1.765293$

0.551661

$-0.391277$

1. 935677

2. 415758

4. 760710

3. 783775

6.134729

6.566808

8.874765

7.931826

10.243784

10.662862

$-8.235294$

$-9.157232$

$-6.785278$

$-6.356199$

$-4.061245$

$-4.991183$

$-2.692231$

$-2.234149$

0.067804
$-1.173017$

$-1.179017$

$-1.011017$

$-0.842017$

$-0.586017$

$-0.662017$

$-0.470017$

$-0.420017$

$-0.559017$

$-0.368017$

$-0.532017$

$-0.307017$

$-0.304017$

$-0.290017$

$-0.382017$

$-0.451017$

$-0.771017$

$-0.605017$

$-0.920017$

$-0.917017$

$-1.030017$

$-1.053017$

$-0.863017$

$-0.726017$

$-0.548017$

$-0.569017$

$-0.414017$

$-0.246017$

$-0.192017$

$-0.111017$

$-0.161017$

$-0.030017$

$-0.250017$

$-0.106017$

$-0.344017$

$-0.337017$

$-0.616017$

$-0.476017$

$-0.771017$

$-0.787017$

$-0.921017$

$-0.962017$

$-0.756017$

$-0.622017$

$-0.419017$

$-0.451017$

$-0.236017$

$-0.047017$

0.085983

0.162983

0.141983

0.291983

$-0.047017$

0.155983

$-0.186017$

$-0.183017$

$-0.490017$

$-0.350017$

$-0.652017$

$-0.685017$

$-0.845017$

$-0.901017$

$-0.665017$

$-0.545017$

$-0.302017$

$-0.358017$

$-0.085017$

0.119983

0.421983 


\begin{tabular}{|c|c|c|c|}
\hline $\mathrm{C}$ & -2.083924 & -0.902136 & 0.439983 \\
\hline $\mathrm{C}$ & -2.820060 & 1.422821 & 0.492983 \\
\hline $\mathrm{C}$ & -1.496088 & 1.900899 & 0.579983 \\
\hline $\mathrm{C}$ & -2.274224 & 4.221853 & 0.174983 \\
\hline $\mathrm{C}$ & -1.183166 & 3.235917 & 0.363983 \\
\hline $\mathrm{C}$ & -1.948303 & 5.580872 & -0.017017 \\
\hline $\mathrm{C}$ & -0.651328 & 5.995948 & -0.065017 \\
\hline $\mathrm{C}$ & -1.383466 & 8.363905 & -0.389017 \\
\hline $\mathrm{C}$ & -0.330412 & 7.433967 & -0.269017 \\
\hline $\mathrm{C}$ & -1.084547 & 9.734923 & -0.563017 \\
\hline $\mathrm{C}$ & 0.250428 & 10.164001 & -0.620017 \\
\hline $\mathrm{C}$ & -2.401465 & -8.733154 & -0.797017 \\
\hline $\mathrm{C}$ & -1.344411 & -9.652092 & -0.865017 \\
\hline $\mathrm{C}$ & -2.108550 & -7.289137 & -0.614017 \\
\hline $\mathrm{C}$ & -0.769575 & -6.853059 & -0.520017 \\
\hline $\mathrm{C}$ & -1.554709 & -4.567105 & -0.236017 \\
\hline $\mathrm{C}$ & -0.491655 & -5.488043 & -0.335017 \\
\hline $\mathrm{C}$ & -1.263793 & -3.144088 & 0.013983 \\
\hline $\mathrm{C}$ & 0.063183 & -2.731010 & 0.111983 \\
\hline $\mathrm{C}$ & -0.711947 & -0.512055 & 0.738983 \\
\hline $\mathrm{C}$ & 0.380106 & -1.409991 & 0.392983 \\
\hline C & -0.389034 & 0.980964 & 0.806983 \\
\hline $\mathrm{C}$ & 0.976941 & 1.398044 & 0.508983 \\
\hline $\mathrm{C}$ & 0.142809 & 3.655995 & 0.282983 \\
\hline $\mathrm{C}$ & 1.210862 & 2.755057 & 0.314983 \\
\hline $\mathrm{C}$ & 0.408726 & 5.082010 & 0.058983 \\
\hline $\mathrm{C}$ & 1.806700 & 5.524092 & -0.072017 \\
\hline $\mathrm{C}$ & 1.019562 & 7.873046 & -0.346017 \\
\hline $\mathrm{C}$ & 2.088616 & 6.953109 & -0.263017 \\
\hline $\mathrm{C}$ & 1.303482 & 9.244063 & -0.521017 \\
\hline $\mathrm{C}$ & 2.637456 & 9.680141 & -0.603017 \\
\hline $\mathrm{C}$ & -0.010437 & -9.223014 & -0.778017 \\
\hline $\mathrm{C}$ & 1.043617 & -10.142953 & -0.847017 \\
\hline $\mathrm{C}$ & 0.294479 & -7.776996 & -0.612017 \\
\hline $\mathrm{C}$ & 1.586454 & -7.358921 & -0.553017 \\
\hline $\mathrm{C}$ & 0.853320 & -5.057964 & -0.268017 \\
\hline $\mathrm{C}$ & 1.897374 & -5.994903 & -0.395017 \\
\hline $\mathrm{C}$ & 1.161240 & -3.700946 & -0.080017 \\
\hline $\mathrm{C}$ & 2.498215 & -3.264867 & -0.066017 \\
\hline $\mathrm{C}$ & 1.699078 & -0.926914 & 0.316983 \\
\hline $\mathrm{C}$ & 2.796134 & -1.896850 & 0.081983 \\
\hline $\mathrm{C}$ & 1.985999 & 0.422103 & 0.369983 \\
\hline $\mathrm{C}$ & 3.380972 & 0.870184 & 0.160983 \\
\hline $\mathrm{C}$ & 2.603834 & 3.229139 & 0.110983 \\
\hline $\mathrm{C}$ & 3.660888 & 2.308201 & 0.069983 \\
\hline $\mathrm{C}$ & 2.857754 & 4.597154 & -0.045017 \\
\hline $\mathrm{C}$ & 4.251726 & 5.072235 & -0.198017 \\
\hline $\mathrm{C}$ & 3.428591 & 7.385187 & -0.374017 \\
\hline $\mathrm{C}$ & 4.499646 & 6.452250 & -0.339017 \\
\hline $\mathrm{C}$ & 3.699510 & 8.761203 & -0.534017 \\
\hline $\mathrm{C}$ & 5.026485 & 9.201281 & -0.633017 \\
\hline $\mathrm{C}$ & 2.380593 & -9.718874 & -0.780017 \\
\hline $\mathrm{C}$ & 3.416647 & -10.655814 & -0.844017 \\
\hline $\mathrm{C}$ & 2.690512 & -8.347856 & -0.649017 \\
\hline $\mathrm{C}$ & 4.040487 & -7.918777 & -0.605017 \\
\hline $\mathrm{C}$ & 3.244349 & -5.562824 & -0.365017 \\
\hline $\mathrm{C}$ & 4.354407 & -6.546759 & -0.483017 \\
\hline $\mathrm{C}$ & 3.536269 & -4.197807 & -0.222017 \\
\hline $\mathrm{C}$ & 4.940242 & -3.733724 & -0.240017 \\
\hline $\mathrm{C}$ & 4.124108 & -1.436772 & -0.007017 \\
\hline $\mathrm{C}$ & 5.219165 & -2.414708 & -0.158017 \\
\hline $\mathrm{C}$ & 4.419027 & -0.061755 & 0.021983 \\
\hline $\mathrm{C}$ & 5.827999 & 0.414328 & -0.118017 \\
\hline $\mathrm{C}$ & 5.054862 & 2.759282 & -0.103017 \\
\hline $\mathrm{C}$ & 6.103917 & 1.811344 & -0.162017 \\
\hline $\mathrm{C}$ & 5.316781 & 4.140298 & -0.208017 \\
\hline $\mathrm{C}$ & 6.644754 & 4.591375 & -0.333017 \\
\hline C & 5.829619 & 6.903328 & -0.457017 \\
\hline $\mathrm{C}$ & 6.901673 & 5.975390 & -0.445017 \\
\hline $\mathrm{C}$ & 6.089538 & 8.284343 & -0.593017 \\
\hline $\mathrm{C}$ & 7.410512 & 8.732420 & -0.693017 \\
\hline
\end{tabular}




\begin{tabular}{|c|c|c|c|}
\hline C & 4.755623 & -10.244735 & -0.786017 \\
\hline $\mathrm{C}$ & 5.773679 & -11.197676 & -0.837017 \\
\hline $\mathrm{C}$ & 5.076543 & -8.874716 & -0.676017 \\
\hline C & 6.428519 & -8.465637 & -0.632017 \\
\hline C & 5.709382 & -6.115679 & -0.466017 \\
\hline C & 6.745438 & -7.080619 & -0.545017 \\
\hline C & 6.026300 & -4.731661 & -0.360017 \\
\hline C & 7.363274 & -4.275582 & -0.381017 \\
\hline $\mathrm{C}$ & 6.618136 & -1.927626 & -0.218017 \\
\hline C & 7.656192 & -2.879565 & -0.320017 \\
\hline C & 6.887054 & -0.527610 & -0.207017 \\
\hline C & 8.221027 & -0.058532 & -0.279017 \\
\hline $\mathrm{C}$ & 7.437890 & 2.269422 & -0.273017 \\
\hline $\mathrm{C}$ & 8.497945 & 1.337484 & -0.302017 \\
\hline $\mathrm{C}$ & 7.710809 & 3.660438 & -0.346017 \\
\hline C & 9.044782 & 4.121516 & -0.447017 \\
\hline C & 8.228646 & 6.436468 & -0.554017 \\
\hline $\mathrm{C}$ & 9.304700 & 5.519531 & -0.549017 \\
\hline C & 8.477565 & 7.821483 & -0.670017 \\
\hline $\mathrm{C}$ & 9.788538 & 8.287560 & -0.766017 \\
\hline $\mathrm{C}$ & 7.123657 & -10.826597 & -0.779017 \\
\hline C & 8.070718 & -11.859541 & -0.840017 \\
\hline C & 7.462576 & -9.443577 & -0.671017 \\
\hline $\mathrm{C}$ & 8.796551 & -9.003499 & -0.586017 \\
\hline $\mathrm{C}$ & 8.098414 & -6.668539 & -0.524017 \\
\hline $\mathrm{C}$ & 9.104471 & -7.650481 & -0.510017 \\
\hline $\mathrm{C}$ & 8.418328 & -5.205521 & -0.494017 \\
\hline $\mathrm{C}$ & 9.734300 & -4.719444 & -0.620017 \\
\hline $\mathrm{C}$ & 8.998167 & -2.447487 & -0.388017 \\
\hline C & 10.009223 & -3.411428 & -0.572017 \\
\hline $\mathrm{C}$ & 9.293081 & -0.979469 & -0.320017 \\
\hline $\mathrm{C}$ & 10.608052 & -0.486392 & -0.275017 \\
\hline C & 9.842918 & 1.792563 & -0.354017 \\
\hline $\mathrm{C}$ & 10.869975 & 0.823623 & -0.291017 \\
\hline $\mathrm{C}$ & 10.116836 & 3.191579 & -0.451017 \\
\hline $\mathrm{C}$ & 11.428807 & 3.697656 & -0.580017 \\
\hline $\mathrm{C}$ & 10.638672 & 6.001609 & -0.656017 \\
\hline $\mathrm{C}$ & 11.681727 & 5.060670 & -0.673017 \\
\hline $\mathrm{C}$ & 10.877590 & 7.405623 & -0.755017 \\
\hline $\mathrm{C}$ & 12.157556 & 7.974698 & -0.841017 \\
\hline $\mathrm{H}$ & -12.951549 & -7.308772 & -1.299017 \\
\hline $\mathrm{H}$ & -11.055457 & -8.865661 & -1.308017 \\
\hline $\mathrm{H}$ & -12.613688 & -4.934752 & -1.031017 \\
\hline $\mathrm{H}$ & -12.244806 & -2.919731 & -0.614017 \\
\hline $\mathrm{H}$ & -11.819937 & -0.669706 & -0.404017 \\
\hline $\mathrm{H}$ & -11.376056 & 1.352320 & -0.767017 \\
\hline $\mathrm{H}$ & -10.899187 & 3.600348 & -0.724017 \\
\hline $\mathrm{H}$ & -10.498304 & 5.593372 & -0.240017 \\
\hline $\mathrm{H}$ & -10.010435 & 7.818400 & -0.381017 \\
\hline $\mathrm{H}$ & -9.565550 & 9.786426 & -0.809017 \\
\hline $\mathrm{H}$ & -8.943685 & 12.089463 & -1.044017 \\
\hline $\mathrm{H}$ & -6.592725 & 12.783600 & -1.037017 \\
\hline $\mathrm{H}$ & -8.675433 & -9.275522 & -1.183017 \\
\hline $\mathrm{H}$ & -4.242693 & 12.231738 & -0.910017 \\
\hline $\mathrm{H}$ & -6.320407 & -9.723384 & -1.092017 \\
\hline $\mathrm{H}$ & -1.892663 & 11.714875 & -0.815017 \\
\hline $\mathrm{H}$ & -3.948379 & -10.212245 & -1.034017 \\
\hline $\mathrm{H}$ & 0.471366 & 11.218014 & -0.755017 \\
\hline $\mathrm{H}$ & -1.560350 & -10.707105 & -0.992017 \\
\hline $\mathrm{H}$ & 2.850395 & 10.735153 & -0.733017 \\
\hline $\mathrm{H}$ & 0.821679 & -11.199966 & -0.957017 \\
\hline $\mathrm{H}$ & 5.234423 & 10.259293 & -0.748017 \\
\hline $\mathrm{H}$ & 3.179709 & -11.709828 & -0.937017 \\
\hline $\mathrm{H}$ & 7.610450 & 9.794432 & -0.791017 \\
\hline $\mathrm{H}$ & 5.506741 & -12.246691 & -0.922017 \\
\hline $\mathrm{H}$ & 9.961475 & 9.356570 & -0.849017 \\
\hline $\mathrm{H}$ & 9.136709 & -11.705479 & -0.823017 \\
\hline $\mathrm{H}$ & 7.755778 & -12.893560 & -0.920017 \\
\hline $\mathrm{H}$ & 9.631590 & -9.683450 & -0.571017 \\
\hline $\mathrm{H}$ & 10.149457 & -7.402419 & -0.424017 \\
\hline $\mathrm{H}$ & 10.569338 & -5.380395 & -0.784017 \\
\hline
\end{tabular}




$\begin{array}{rrrr}\mathrm{H} & 11.042207 & -3.134367 & -0.702017 \\ \mathrm{H} & 11.459090 & -1.145343 & -0.212017 \\ \mathrm{H} & 11.911960 & 1.087684 & -0.241017 \\ \mathrm{H} & 12.297844 & 3.065706 & -0.636017 \\ \mathrm{H} & 12.715710 & 5.345731 & -0.775017 \\ \mathrm{H} & 13.074589 & 7.409752 & -0.825017 \\ \mathrm{H} & 12.280493 & 9.048705 & -0.917017 \\ \mathrm{O} & -0.541987 & 0.169955 & 2.021983 \\ \mathrm{C} & -1.556803 & -2.966105 & 3.352983 \\ \mathrm{C} & -0.888858 & -2.023066 & 4.023983 \\ \mathrm{C} & -3.020796 & -3.083191 & 3.259983 \\ \mathrm{H} & -0.987757 & -3.746072 & 2.844983 \\ \mathrm{C} & -3.593723 & -4.336224 & 3.018983 \\ \mathrm{C} & -4.974714 & -4.481305 & 2.931983 \\ \mathrm{C} & -5.800779 & -3.368353 & 3.069983 \\ \mathrm{C} & -5.239853 & -2.111321 & 3.289983 \\ \mathrm{C} & -3.859861 & -1.968240 & 3.379983 \\ \mathrm{H} & -2.947672 & -5.203186 & 2.897983 \\ \mathrm{H} & -5.404657 & -5.458330 & 2.739983 \\ \mathrm{H} & -6.877773 & -3.476416 & 2.983983 \\ \mathrm{H} & -5.878904 & -1.237358 & 3.369983 \\ \mathrm{H} & -3.423919 & -0.979214 & 3.501983 \\ \mathrm{C} & 0.611140 & -1.999978 & 4.110983 \\ \mathrm{H} & -1.437902 & -1.270098 & 4.587983 \\ \mathrm{H} & 0.945159 & -2.323958 & 5.103983 \\ \mathrm{H} & 1.020182 & -2.709954 & 3.381983 \\ \mathrm{C} & 1.226060 & -0.627942 & 3.829983 \\ \mathrm{C} & 3.096962 & 1.049168 & 3.841983 \\ \mathrm{C} & 1.925932 & 1.556099 & 4.392983 \\ \mathrm{H} & 0.493034 & -0.173985 & 2.765983 \\ \mathrm{~S} & 2.933058 & -0.586842 & 3.405983 \\ \mathrm{C} & 0.909988 & 0.603040 & 4.459983 \\ \mathrm{H} & -0.083023 & 0.798981 & 4.853983 \\ \mathrm{C} & 4.372918 & 1.794242 & 3.597983 \\ \mathrm{H} & 4.155861 & 2.777230 & 3.171983 \\ \mathrm{H} & 4.904909 & 1.945274 & 4.541983 \\ \mathrm{H} & 5.031950 & 1.255281 & 2.913983 \\ \mathrm{H} & 1.825872 & 2.585093 & 4.717983 \\ & & & \end{array}$

\section{PD3}

C $\quad-11.289440$

C $\quad-10.124702$

C $\quad-11.226190$

C $\quad-9.982902$

C $\quad-11.005658$

C $\quad-9.871059$

C -10.898203

C $\quad-9.645105$

C $\quad-10.602765$

C $\quad-9.504883$

C -10.458430

C $\quad-9.200729$

C $\quad-10.128217$

C $\quad-9.028580$

C $\quad-9.972962$

C $\quad-8.696598$

C $\quad-9.615730$

C $\quad-8.524896$

C $\quad-9.409760$

C $\quad-8.122670$

C $\quad-8.877431$

$-7.714215$

$-8.796487$

$-7.529633$

$-8.587046$

$-7.426022$

$-8.484202$

$-7.159368$

$-8.221855$

$-7.039998$

$-1.062309$

$-1.090374$

$-0.912901$

$-0.782170$

$-0.582776$

$-0.627337$

$-0.475720$

$-0.404847$

$-0.492258$

$-0.340951$

$-0.446356$

$-0.240040$

$-0.166332$

$-0.192097$

$-0.205978$

$-0.272826$

$-0.488350$

$-0.383697$

$-0.603495$

$-0.626349$

$-0.967956$

$-1.006347$

$-0.810403$

$-0.683637$

$-0.513241$

$-0.527158$

$-0.388612$

$-0.227780$

$-0.179072$

$-0.109541$ 


\begin{tabular}{|c|c|c|c|}
\hline $\mathrm{C}$ & -8.060310 & 1.706400 & -0.132000 \\
\hline $\mathrm{C}$ & -6.774944 & 2.293613 & -0.015980 \\
\hline $\mathrm{C}$ & -7.725005 & 4.562029 & -0.159124 \\
\hline $\mathrm{C}$ & -6.599780 & 3.708267 & -0.060609 \\
\hline $\mathrm{C}$ & -7.556046 & 5.973560 & -0.214507 \\
\hline $\mathrm{C}$ & -6.256654 & 6.528323 & -0.217739 \\
\hline $\mathrm{C}$ & -7.211098 & 8.775486 & -0.412679 \\
\hline $\mathrm{C}$ & -6.084989 & 7.926742 & -0.323614 \\
\hline $\mathrm{C}$ & -7.019574 & 10.176340 & -0.535500 \\
\hline $\mathrm{C}$ & -5.728657 & 10.712480 & -0.574471 \\
\hline $\mathrm{C}$ & -6.457919 & -8.414105 & -0.888276 \\
\hline $\mathrm{C}$ & -5.304561 & -9.209304 & -0.943087 \\
\hline $\mathrm{C}$ & -6.360128 & -7.014405 & -0.720322 \\
\hline $\mathrm{C}$ & -5.014943 & -6.392610 & -0.586141 \\
\hline $\mathrm{C}$ & -6.074418 & -4.192054 & -0.371678 \\
\hline $\mathrm{C}$ & -4.912461 & -4.999496 & -0.400112 \\
\hline $\mathrm{C}$ & -5.953232 & -2.799428 & -0.201228 \\
\hline $\mathrm{C}$ & -4.688956 & -2.213928 & -0.016707 \\
\hline $\mathrm{C}$ & -5.708192 & 0.012609 & 0.067309 \\
\hline $\mathrm{C}$ & -4.567871 & -0.808419 & 0.144093 \\
\hline $\mathrm{C}$ & -5.585813 & 1.414047 & 0.116478 \\
\hline C & -4.243401 & 2.030974 & 0.272633 \\
\hline $\mathrm{C}$ & -5.299247 & 4.283117 & -0.010610 \\
\hline $\mathrm{C}$ & -4.098607 & 3.417194 & 0.144371 \\
\hline $\mathrm{C}$ & -5.126577 & 5.685932 & -0.113985 \\
\hline $\mathrm{C}$ & -3.825389 & 6.252306 & -0.122280 \\
\hline $\mathrm{C}$ & -4.782752 & 8.482674 & -0.355341 \\
\hline $\mathrm{C}$ & -3.651240 & 7.646359 & -0.260372 \\
\hline $\mathrm{C}$ & -4.611142 & 9.878991 & -0.489475 \\
\hline $\mathrm{C}$ & -3.324041 & 10.429767 & -0.546503 \\
\hline $\mathrm{C}$ & -4.032231 & -8.641320 & -0.834783 \\
\hline $\mathrm{C}$ & -2.886527 & -9.446835 & -0.905842 \\
\hline $\mathrm{C}$ & -3.900983 & -7.171102 & -0.643974 \\
\hline C & -2.613486 & -6.605114 & -0.526671 \\
\hline $\mathrm{C}$ & -3.638170 & -4.411166 & -0.245490 \\
\hline $\mathrm{C}$ & -2.477909 & -5.220111 & -0.316293 \\
\hline $\mathrm{C}$ & -3.541649 & -3.011808 & -0.031314 \\
\hline $\mathrm{C}$ & -2.204645 & -2.414429 & 0.170869 \\
\hline $\mathrm{C}$ & -3.229539 & -0.208068 & 0.348530 \\
\hline $\mathrm{C}$ & -2.093515 & -1.069773 & 0.392026 \\
\hline $\mathrm{C}$ & -3.065301 & 1.140160 & 0.394391 \\
\hline $\mathrm{C}$ & -1.747309 & 1.746311 & 0.447066 \\
\hline $\mathrm{C}$ & -2.794178 & 3.991810 & 0.154857 \\
\hline $\mathrm{C}$ & -1.603900 & 3.121355 & 0.298525 \\
\hline $\mathrm{C}$ & -2.628959 & 5.379512 & 0.001469 \\
\hline $\mathrm{C}$ & -1.381011 & 5.925240 & -0.057393 \\
\hline $\mathrm{C}$ & -2.351132 & 8.212331 & -0.317034 \\
\hline $\mathrm{C}$ & -1.208169 & 7.391434 & -0.235629 \\
\hline $\mathrm{C}$ & -2.193801 & 9.608496 & -0.470206 \\
\hline $\mathrm{C}$ & -0.909266 & 10.169511 & -0.554499 \\
\hline $\mathrm{C}$ & -1.607405 & -8.886996 & -0.803637 \\
\hline $\mathrm{C}$ & -0.463223 & -9.692649 & -0.884699 \\
\hline $\mathrm{C}$ & -1.463205 & -7.421754 & -0.607216 \\
\hline $\mathrm{C}$ & -0.176070 & -6.853531 & -0.504151 \\
\hline $\mathrm{C}$ & -1.187687 & -4.666674 & -0.174654 \\
\hline $\mathrm{C}$ & -0.037814 & -5.470238 & -0.292418 \\
\hline $\mathrm{C}$ & -1.045332 & -3.221047 & 0.101173 \\
\hline $\mathrm{C}$ & 0.242568 & -2.683825 & 0.203342 \\
\hline $\mathrm{C}$ & -0.774167 & -0.504783 & 0.820564 \\
\hline $\mathrm{C}$ & 0.408789 & -1.337213 & 0.424040 \\
\hline $\mathrm{C}$ & -0.610803 & 0.949698 & 0.525953 \\
\hline $\mathrm{C}$ & 0.666763 & 1.501187 & 0.418875 \\
\hline $\mathrm{C}$ & -0.335518 & 3.675003 & 0.225063 \\
\hline $\mathrm{C}$ & 0.807865 & 2.881275 & 0.258827 \\
\hline $\mathrm{C}$ & -0.216775 & 5.123054 & 0.049101 \\
\hline $\mathrm{C}$ & 1.112545 & 5.702707 & -0.095957 \\
\hline $\mathrm{C}$ & 0.090011 & 7.963044 & -0.330728 \\
\hline $\mathrm{C}$ & 1.247650 & 7.154075 & -0.276305 \\
\hline $\mathrm{C}$ & 0.232168 & 9.357584 & -0.495172 \\
\hline $\mathrm{C}$ & 1.514172 & 9.923136 & -0.606258 \\
\hline $\mathrm{C}$ & 0.819262 & -9.132502 & -0.794038 \\
\hline
\end{tabular}




\begin{tabular}{|c|c|c|c|}
\hline C & 1.959332 & -9.940775 & -0.882465 \\
\hline $\mathrm{C}$ & 0.975700 & -7.665286 & -0.603363 \\
\hline $\mathrm{C}$ & 2.219444 & -7.122840 & -0.530476 \\
\hline $\mathrm{C}$ & 1.258181 & -4.911869 & -0.200674 \\
\hline C & 2.391629 & -5.739281 & -0.341691 \\
\hline C & 1.428793 & -3.542784 & 0.010680 \\
\hline C & 2.718710 & -2.972140 & 0.041142 \\
\hline$c$ & 1.693918 & -0.734206 & 0.373873 \\
\hline C & 2.882609 & -1.592602 & 0.193459 \\
\hline $\mathrm{C}$ & 1.823020 & 0.621881 & 0.361749 \\
\hline C & 3.167406 & 1.221309 & 0.178823 \\
\hline $\mathrm{C}$ & 2.153769 & 3.487319 & 0.0 \\
\hline $\mathrm{C}$ & 3.282466 & 2.685851 & 0.051907 \\
\hline $\mathrm{C}$ & 2.252088 & 4.884334 & -0.076245 \\
\hline $\mathrm{C}$ & 3.589563 & 5.497983 & -0.235805 \\
\hline $\mathrm{C}$ & 2.534999 & 7.717702 & -0.405217 \\
\hline C & 3.694771 & 6.896275 & -0.386088 \\
\hline C & 2.662764 & 9.114034 & -0.568622 \\
\hline C & 3.936895 & 9.683692 & -0.701096 \\
\hline C & 3.246542 & -9.385141 & -0.814177 \\
\hline C & 4.370977 & -10.210781 & -0.913285 \\
\hline C & 3.417364 & -7.993916 & -0.651994 \\
\hline C & 4.717318 & -7.432500 & -0.611399 \\
\hline $\mathrm{C}$ & 3.688825 & -5.176715 & -0.303125 \\
\hline C & 4.891967 & -6.039617 & -0.459334 \\
\hline C & 3.843252 & -3.792852 & -0.132487 \\
\hline $\mathrm{C}$ & 5.192498 & -3.188100 & -0.167802 \\
\hline $\mathrm{C}$ & 4.148792 & -0.988742 & 0.079725 \\
\hline $\mathrm{C}$ & 5.335977 & -1.847981 & -0.086050 \\
\hline C & 4.293706 & 0.410873 & 0.068396 \\
\hline C & 5.642838 & 1.029568 & -0.102019 \\
\hline $\mathrm{C}$ & 4.625148 & 3.279729 & -0.126073 \\
\hline C & 5.768889 & 2.446047 & -0.178231 \\
\hline C & 4.744318 & 4.679962 & -0.249516 \\
\hline C & 6.017316 & 5.263688 & -0.399578 \\
\hline C & 4.970037 & 7.479116 & -0.532378 \\
\hline C & 6.130301 & 6.665119 & -0.534643 \\
\hline $\mathrm{C}$ & 5.086248 & 8.878097 & -0.685181 \\
\hline $\mathrm{C}$ & 6.352063 & 9.455562 & -0.828121 \\
\hline $\mathrm{C}$ & 5.662126 & -9.667534 & -0.866468 \\
\hline C & 6.770643 & -10.509601 & -0.965709 \\
\hline C & 5.843472 & -8.275150 & -0.725593 \\
\hline C & 7.147583 & -7.732027 & -0.696764 \\
\hline C & 6.196187 & -5.473079 & -0.456906 \\
\hline C & 7.3 & -6.324259 & -0 \\
\hline C & 6.372344 & -4.067434 & -0.329485 \\
\hline $\mathrm{C}$ & 7.653407 & -3.475045 & -0.379287 \\
\hline C & 6.672982 & -1.218318 & -0.186168 \\
\hline $\mathrm{C}$ & 7.802290 & -2.057738 & -0.315656 \\
\hline $\mathrm{C}$ & 6.793601 & 0.201748 & -0.197064 \\
\hline C & 8.069269 & 0.804825 & -0.306438 \\
\hline $\mathrm{C}$ & 7.047026 & 3.037618 & -0.319494 \\
\hline $\mathrm{C}$ & 8.197732 & 2.221167 & -0.355523 \\
\hline $\mathrm{C}$ & 7.173303 & 4.447024 & -0.421138 \\
\hline C & 8.450736 & 5.040867 & -0.562853 \\
\hline C & 7.400978 & 7.257578 & 34189 \\
\hline C & 8.564728 & 6.455154 & -0.696726 \\
\hline C & 7.504652 & 8.657491 & -0.827638 \\
\hline C & 8.758322 & 9.252398 & -0.972831 \\
\hline C & 8.077023 & -10.002782 & -0.927963 \\
\hline C & 9.123639 & -10.929774 & -1.047764 \\
\hline C & 8.275634 & -8.597014 & -0.785317 \\
\hline $\mathrm{C}$ & 9.558720 & -8.023979 & -0.713841 \\
\hline $\mathrm{C}$ & 8.627396 & -5.776762 & -0.581103 \\
\hline C & 9.728946 & -6.649450 & -0.609547 \\
\hline $\mathrm{C}$ & 8.795455 & -4.289268 & -0.533681 \\
\hline C & 0579 & -3.669437 & -0.690703 \\
\hline C & 9.088390 & -1.488861 & -0.419645 \\
\hline C & 10.189278 & -2.340848 & -0.638951 \\
\hline C & 9.229819 & 0.000271 & -0.361537 \\
\hline $\mathrm{C}$ & 10.487551 & 0.627129 & -0.342672 \\
\hline
\end{tabular}




\begin{tabular}{|c|c|c|c|}
\hline C & 9.487001 & 2.810813 & -0.445390 \\
\hline $\mathrm{C}$ & 10.611070 & 1.955706 & -0.382640 \\
\hline $\mathrm{C}$ & 9.612777 & 4.226744 & -0.578446 \\
\hline $\mathrm{C}$ & 10.862105 & 4.858844 & -0.759093 \\
\hline $\mathrm{C}$ & 9.839410 & 7.067342 & -0.852984 \\
\hline $\mathrm{C}$ & 10.971994 & 6.237159 & -0.886512 \\
\hline $\mathrm{C}$ & 9.931660 & 8.485711 & -0.984309 \\
\hline $\mathrm{C}$ & 11.143632 & 9.179294 & -1.124434 \\
\hline $\mathrm{H}$ & -12.250870 & -8.594384 & -1.160419 \\
\hline $\mathrm{H}$ & -10.199022 & -9.937222 & -1.210487 \\
\hline $\mathrm{H}$ & -12.166539 & -6.197404 & -0.907004 \\
\hline $\mathrm{H}$ & -12.006079 & -4.132873 & -0.632602 \\
\hline $\mathrm{H}$ & -11.819674 & -1.850058 & -0.437000 \\
\hline $\mathrm{H}$ & -11.597710 & 0.222923 & -0.676054 \\
\hline $\mathrm{H}$ & -11.350400 & 2.508598 & -0.602786 \\
\hline $\mathrm{H}$ & -11.140788 & 4.530058 & -0.101433 \\
\hline $\mathrm{H}$ & -10.881689 & 6.796763 & -0.178127 \\
\hline $\mathrm{H}$ & -10.643742 & 8.820027 & -0.498249 \\
\hline $\mathrm{H}$ & -10.258719 & 11.178965 & -0.682083 \\
\hline $\mathrm{H}$ & -7.989346 & 12.101685 & -0.721105 \\
\hline $\mathrm{H}$ & -7.787938 & -10.088373 & -1.132350 \\
\hline $\mathrm{H}$ & -5.593485 & 11.784497 & -0.675945 \\
\hline $\mathrm{H}$ & -5.398860 & -10.281822 & -1.077743 \\
\hline $\mathrm{H}$ & -3.201711 & 11.501992 & -0.659384 \\
\hline $\mathrm{H}$ & -2.991601 & -10.517182 & -1.047341 \\
\hline $\mathrm{H}$ & -0.797989 & 11.241534 & -0.678402 \\
\hline $\mathrm{H}$ & -0.571322 & -10.762667 & -1.025990 \\
\hline $\mathrm{H}$ & 1.617941 & 10.995318 & -0.734417 \\
\hline $\mathrm{H}$ & 1.844578 & -11.011467 & -1.014580 \\
\hline $\mathrm{H}$ & 4.034344 & 10.756957 & -0.825777 \\
\hline $\mathrm{H}$ & 4.241077 & -11.281640 & -1.0 \\
\hline $\mathrm{H}$ & 6.440712 & 10.530716 & -0.944608 \\
\hline $\mathrm{H}$ & 6.611189 & -11.577928 & -1.076047 \\
\hline $\mathrm{H}$ & 8.819409 & 10.331362 & -1.077815 \\
\hline $\mathrm{H}$ & 10.167717 & -10.666081 & -1.057829 \\
\hline $\mathrm{H}$ & 8.915049 & -11.987897 & -1.153844 \\
\hline $\mathrm{H}$ & 10.459141 & -8.615537 & -0.730346 \\
\hline $\mathrm{H}$ & 10.744318 & -6.295919 & -0.537881 \\
\hline $\mathrm{H}$ & 10.944460 & -4.239096 & -0.886020 \\
\hline $\mathrm{H}$ & 11.182883 & -1.955545 & -0.799704 \\
\hline $\mathrm{H}$ & 11.402347 & 0.059961 & -0.283331 \\
\hline $\mathrm{H}$ & 11.620604 & 2.327795 & -0.3 \\
\hline $\mathrm{H}$ & 11.788447 & 4.316540 & -0.833998 \\
\hline $\mathrm{H}$ & 11.968102 & 6.623607 & -1.025950 \\
\hline $\mathrm{H}$ & 12.112998 & 8.710342 & -1.132018 \\
\hline $\mathrm{H}$ & 11.154314 & 10.258354 & -1.224095 \\
\hline 0 & -0.852184 & -0.661766 & 2.256703 \\
\hline $\mathrm{C}$ & 0.950090 & -3.560600 & 3.328780 \\
\hline $\mathrm{C}$ & 0.977500 & -2.419552 & 4.026781 \\
\hline $\mathrm{C}$ & -0.239712 & -4.411104 & 3.151978 \\
\hline $\mathrm{H}$ & 1.878671 & -3.935756 & 2.894849 \\
\hline $\mathrm{C}$ & -0.088466 & -5.794127 & 2.981374 \\
\hline $\mathrm{C}$ & -1.199742 & -6.623215 & 7251 \\
\hline $\mathrm{C}$ & -2.483994 & -6.081905 & 2.908190 \\
\hline $\mathrm{C}$ & -2.647451 & -4.704917 & 3.043736 \\
\hline $\mathrm{C}$ & -1.537283 & -3.873769 & 3.154496 \\
\hline $\mathrm{H}$ & 0.911144 & -6.220122 & 2.948411 \\
\hline $\mathrm{H}$ & -1.063762 & -7.692130 & 2.738470 \\
\hline $\mathrm{H}$ & -3.351017 & -6.727258 & 2.810868 \\
\hline $\mathrm{H}$ & -3.644513 & -4.274087 & 3.044607 \\
\hline $\mathrm{H}$ & -1.666052 & -2.796117 & 3.208772 \\
\hline $\mathrm{C}$ & 2.208290 & -1.580314 & 4.230372 \\
\hline $\mathrm{H}$ & 0.072413 & -2.094034 & 4.538460 \\
\hline $\mathrm{H}$ & 2.589319 & -1.742496 & 48994 \\
\hline $\mathrm{H}$ & 2.999938 & -1.911780 & 3.548100 \\
\hline $\mathrm{C}$ & 1.956425 & -0.101653 & 4.051423 \\
\hline $\mathrm{C}$ & 2.250816 & 2.364404 & 3.652263 \\
\hline $\mathrm{C}$ & 0.990133 & 2.032705 & 4.047418 \\
\hline $\mathrm{H}$ & 0.019651 & -0.393983 & 2.615656 \\
\hline $\mathrm{S}$ & 3.251613 & 0.947326 & 3.568175 \\
\hline $\mathrm{C}$ & 0.825345 & 0.632020 & 4.297219 \\
\hline
\end{tabular}




$\begin{array}{rrrr}\mathrm{H} & -0.100678 & 0.211832 & 4.682187 \\ \mathrm{C} & 2.809488 & 3.737119 & 3.406919 \\ \mathrm{H} & 2.050164 & 4.362391 & 2.927459 \\ \mathrm{H} & 3.100824 & 4.203703 & 4.352227 \\ \mathrm{H} & 3.694130 & 3.709487 & 2.765688 \\ \mathrm{H} & 0.196381 & 2.761557 & 4.176560\end{array}$

